\title{
AN EXPLORATION OF STRENGTHS, PROMOTIVE, AND PROTECTIVE FACTORS AMONG JUSTICE INVOLVED YOUTH
}

\author{
by \\ Melissa Harris \\ A thesis submitted to \\ the Faculty of Graduate and Postdoctoral Affairs \\ in partial fulfillment of the requirements for the degree of \\ Master of Arts \\ in \\ Psychology
}

Carleton University

Ottawa, Canada

C2013 Melissa Harris 
Library and Archives

Canada

Published Heritage

Branch

395 Wellington Street

Ottawa ON K1A ON4

Canada
Bibliothèque et

Archives Canada

Direction du

Patrimoine de l'édition

395 , rue Wellington

Ottawa ON K1A ON4

Canada
Your file Votre référence

ISBN: 978-0-494-94322-9

Our file Notre référence

ISBN: $978-0-494-94322-9$
NOTICE:

The author has granted a nonexclusive license allowing Library and Archives Canada to reproduce, publish, archive, preserve, conserve, communicate to the public by telecommunication or on the Internet, loan, distrbute and sell theses worldwide, for commercial or noncommercial purposes, in microform, paper, electronic and/or any other formats.

The author retains copyright ownership and moral rights in this thesis. Neither the thesis nor substantial extracts from it may be printed or otherwise reproduced without the author's permission.
AVIS:

L'auteur a accordé une licence non exclusive permettant à la Bibliothèque et Archives Canada de reproduire, publier, archiver, sauvegarder, conserver, transmettre au public par télécommunication ou par l'Internet, prêter, distribuer et vendre des thèses partout dans le monde, à des fins commerciales ou autres, sur support microforme, papier, électronique et/ou autres formats.

L'auteur conserve la propriété du droit d'auteur et des droits moraux qui protege cette thèse. $\mathrm{Ni}$ la thèse ni des extraits substantiels de celle-ci ne doivent être imprimés ou autrement reproduits sans son autorisation.
In compliance with the Canadian Privacy Act some supporting forms may have been removed from this thesis.

While these forms may be included in the document page count, their removal does not represent any loss of content from the thesis.
Conformément à la loi canadienne sur la protection de la vie privée, quelques formulaires secondaires ont été enlevés de cette thèse.

Bien que ces formulaires aient inclus dans la pagination, il n'y aura aucun contenu manquant. 


\begin{abstract}
Thus far, the trend in correctional psychology has been to quantify the deficits of justiceinvolved youth and to translate these deficits into an estimate of risk to reoffend. However, research on positive and strengths-based approaches is still in its infancy within correctional psychology. The current study examines anonymous, archival data of 59 female and 61 male justice involved youth and adds to the emerging body of literature on the strengths-based mechanisms at work for this population. The extent to which gender influences the number and type of strengths/protective factors on the Youth Level of Service and Case Management Inventory 2.0 (Hoge \& Andrews, 2010), a revised version of a well-established risk assessment tool, as well as the measurement of protective factors in the Youth Assessment Screening Instrument (Orbis Partners, 2007), a newer tool was examined. Results revealed that, in most cases, there were no significant differences between males and females. In addition, the strength domains of the YL/CMI 2.0 and the protective domains of the YASI were compared. The examination of bivariate correlations revealed lower correlations between domains on the YLS/CMI 2.0 and YASI than were expected. Finally, a strengths-based model in which strengths/promotive/protective factors post-dicted criminal history was tested. Results revealed that YLS/CMI 2.0 strengths did not post-dict criminal history over and above YLS/CMI 2.0 criminal need. However, YASI protective factors showed some promise in post-dicting criminal history over and above YASI criminal need. The results of this study suggest that future work examining strengths, promotive, and protective factors needs to be done in order to determine which tool is best at measuring the constructs.
\end{abstract}




\section{Acknowledgments}

I owe many thanks to my advisor Dr. Shelley Brown for guiding me through the process of writing this thesis, constantly pushing me to achieve more than I ever thought I could. I am also incredibly grateful to all of the youth who participated in this study by sharing their personal stories, without them, this project would not have been possible. Thank-you to all of the support staff at the various data collection sites for making the researchers feel welcome and comfortable, as well as all of my lab mates in the Gender and Crime Research Lab for dedicating their time in data collection and entering. I am grateful to my committee members Drs. Kevin Nunes and Joanna Pozzulo for the contribution of their time and suggestions for my thesis. I would also like to thank Etelle Bourassa and all of the Carleton University Psychology Department administrative staff for the endless support they gave to me through this process. I extend my utmost gratitude to both the Social Sciences and Humanities Research Council of Canada and Carleton University for the financial support that made my pursuit in graduate studies possible. My family has been a constant source of support throughout my educational journey, giving me the confidence and love I needed to continue. Finally, I would like to thank my dearest friend and lab mate Celia Geck not only for her academic support, but emotional support at every step of this project-we made it! 


\section{Table of Contents}
Abstract
.ii
Acknowledgements. iii
List of Tables vii
List of Figures viii
Introduction. 1

Defining a Protective Factor 3

Defining a Promotive Factor 4

Defining a Strength Factor 4

The Four Generations of Risk Assessment .....................................................

Gender-Responsive Critique of the Risk-Need-Responsivity Model .......................7

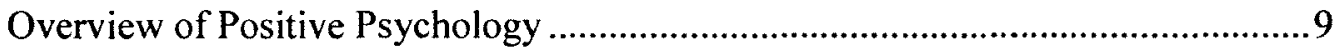

Overview of Resilience Research

Empirical Evidence for Protective and Promotive Factors ............................................. 14

Youth Level of Service and Case Management Inventory 2.0

Youth Assessment and Screening Instrument

Current Study

Research Objective 1: determine if the number and type of strengths as measured on the YLS/CMI and YASI respectively vary as a function of gender 21

Research Objective 2: Determine how well the YASI and YLS/CMI strengths/protective factors - both at a composite level and individual domain levels - correlate with each other (where comparable) for males and females separately. 21 
Research Objective 3: establish if YLS/CMI identified strengths add incrementally to the post-diction of criminal history above and beyond risk (inclusive of criminogenic need factors) and whether or not this relationship is moderated by gender ..... 21

Research Objective 4: establish if YASI identified protective factors add incrementally to the post-diction of criminal history above and beyond risk, as well as whether or not the relationship is moderated by gender .22

Method

Participants.

Procedure. .27

Materials. .29

YLS/CMI 2.0 .29

YLS/CMI 2.0 Psychometic Properties .29

YASI. 31

YASI Prescreen .32

YASI Full Assessment .33

YASI Psychometric Properties. 34

Results.

Data Screening .36

Missing Data .36

Assumptions 39

Controlling for Confounds .40

Descriptive Statistics

Research Objective 1: determine if the number and type of strengths as measured on the YLS/CMI and YASI respectively vary as a function of gender. 53 
Research Objective 2: Determine how well the YASI and YLS/CMI strengths/protective factors - both at a composite level and individual domain levels-correlate with each other (where comparable) for males and females separately. 55

Research Objective 3: establish if YLS/CMI identified strengths add incrementally to the post-diction of criminal history above and beyond risk (inclusive of criminogenic need factors) and whether or not this relationship is moderated by gender ..... .64

Research Objective 4: establish if YASI identified protective factors add incrementally to the post-diction of criminal history above and beyond risk, as well as whether or not the relationship is moderated by gender .65

Discussion. 69

Profile of Strengths and Protective Factors................................................ 70

Comparison of YLS/CMI and YASI Strengths and Protective Factors................72

The Post-diction of YLS/CMI Criminal History .............................................76

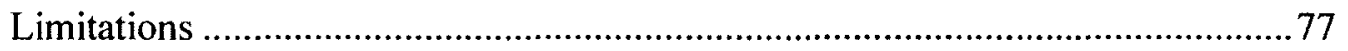

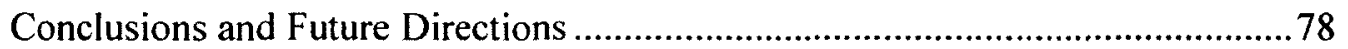






\section{List of Tables}

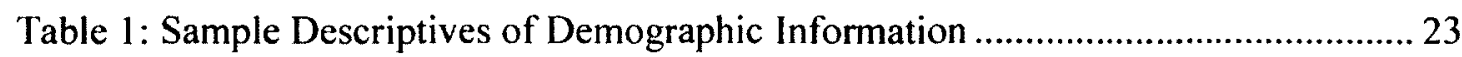

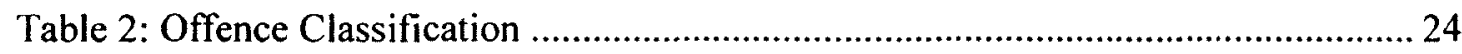

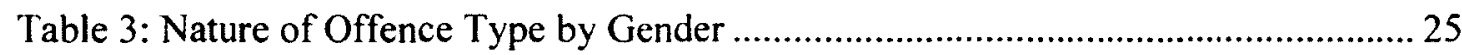

Table 4: Youth Level of Risk and Case Management Index Risk/Need Domain Mean Scores and Corresponding Descriptives by Gender.

Table 5: Youth Assessment and Screening Inventory Risk/Need Domain Mean Scores and Corresponding Descriptives by Gender..... 46

Table 6: Reliability Results: Intra-class Correlation Coefficients For Youth Assessment and Screening Inventory Total Domains 50

Table 7: Reliability Results: Intra-class Correlation Coefficients For Youth Assessment and Screening Inventory Sub-domains 50

Table 8: Summary of Intra-class Correlations and Alphas for Scores on YLS/CMI Subdomains 52

Table 9: Nature of Youth Level of Service Strengths by Gender 54

Table 10: Nature of Youth Assessment Screening Inventory Strengths by Gender......... 56

Table 11: Relationship Between Youth Level of Service and Youth Assessment Screening Inventory Strength Domains 60

Table 12: Gender Moderated Regression Results for Youth Level of Service Strength Total Postdicting Youth Level of Service Legal History Over and Above Criminal Need and Race...66 Table 13: Gender Moderated Regression Results for Youth Assessment and Screening Inventory Protective Total Post-dicting Youth Level of Service Legal History Over and Above Criminal Need and Race 68 


\section{List of Figures}

Figure 1 through 29: Distribution of Youth for YASI Sub-domains and Total Domain Risk

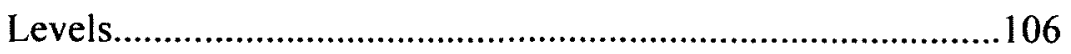




\section{Introduction}

The psychology of criminal conduct "seeks to account for variation in the criminal behaviour of individuals" (Andrews \& Bonta, 2010, p.3.). There are particular subgroups of offenders (e.g. violent and sex offenders) that sometimes require different theories and interventions (Andrews \& Bonta, 2010). In particular, the young offender population has traditionally been considered a vulnerable group, requiring special consideration and separate sets of sanctions and treatments. The Juvenile Delinquents Act (1908-1984) marked the beginning of the treatment of justice involved youth as a unique group of individuals who would benefit from different treatments than their adult counterparts.

Perhaps in response to legislation changes (i.e. the switch from the Young Offenders Act to the Youth Criminal Justice Act, which places more emphasis on rehabilitation and reintegration), research in correctional psychology, in particular research on risk assessment and the treatment of young offenders, has been expanding exponentially. Thus far, the trend in correctional psychology has been to quantify the deficits of justice-involved youth and to translate these deficits into an estimate of risk to reoffend. However, in the late 1990's a sub-discipline, spearheaded by Martin Seligman and labelled positive psychology, emerged. Positive psychology is a philosophical umbrella that aims to examine individual, familial, and community strengths and their corresponding application to various areas of psychological research. Ultimately, positive psychology aims to make normal lives more fulfilling as opposed to striving to alleviate pain and suffering - the mainstay of traditional psychology.

Risk assessment has been well researched within correctional psychology and more specifically within the context of adolescent offending (Dowden \& Andrews, 1999b, Lipsey, 2009; Schwalbe, 2008). However, research on positive psychology and strengths-based approaches is still in its infancy within correctional psychology. Moreover, the terms "strengths" and "protective 
factors" are sometimes used interchangeably. To move the field forward, the next step in assessment research for youthful offenders is to investigate how to, or if we should, incorporate strengths and/or protective factors into what is currently known about risk factors and assessment. For example, Ward (2002) introduced the "Good Lives Model," aimed at inventing a positive approach to the treatment of offenders. In 2006, Ward and Gannon adapted the Good Lives Model into a treatment model for sexual offenders. However, to date, there has yet to be an adaptation of the Good Lives Model for youthful offenders. Furthermore, feminist correctional researchers advocate strengths-based and empowerment based platforms for the treatment of female offenders (Covington \& Bloom, 2007; Hannah-Moffat \& Shaw, 2001; Morton \& Leslie, 2005; Sorbello et al., 2002), but to date, controversy exists as to whether current risk assessment protocols really capture the experience of females and whether or not they accurately assess both risk and strengths/protective factors as they apply to adolescent justice involved females (VanVoorhis, 2012).

The current study adds to the emerging body of literature on the protective mechanisms at work for both male and female justice involved youth. The extent to which gender influences the number and type of strengths/protective factors will be explored. In addition, the measurement of "strengths" in the Youth Level of Service and Case Management Inventory 2.0 (Hoge \& Andrews, 2010), a well-established risk assessment tool, as well as the measurement of protective factors in the Youth Assessment Screening Instrument (Orbis Partners, 2007), a newer tool, will be compared. Each tool uses a slightly different approach to the measurement of strengths. It is also important to clarify if both tools are measuring the same or different constructs.

The forthcoming review first describes existing definitional discrepancies in the literature (e.g., strengths vs. protective vs. promotive factors). Second, the past and present state of risk assessment is explored including feminist critiques of the risk assessment literature. Third, positive 
psychology is reviewed with a specific emphasis on how it relates to both the resiliency and correctional psychology literatures. Fourth, evidence for protective and promotive factors is reviewed within the context of correctional psychology and in particular, youthful offenders. Finally, two tools, the Youth Level of Service and Case Management Inventory 2.0 (YLS/CMI 2.0) and the Youth Assessment and Screening Instrument (YASI), that purportedly capture offender strengths and protective factors, respectively, are introduced. Gender-specific research findings are embedded within each section when applicable.

\section{Defining a Protective Factor}

There has been a fair amount of inconsistency in how the term "protective factor" is used throughout published work. Many times, the term is used to loosely define a characteristic that appears, at face-value, to be positive-a strength. However, for the purposes of this paper, Jessor's 1991 conceptual framework for understanding adolescent problem behaviour will be used. Jessor is well-known for his research on successful adolescent development among youth in high risk settings. Jessor (1991) proposed that when protective factors are present and operating, they can potentially buffer the effects of risk factors. In an analysis of Jessor's 1991 data, junior and senior

high school males and females were classified into no risk, moderate risk, and high risk groups for problem behaviour. Each risk group was cross-classified into high and low protection subgroups. Analysis of variance of involvement in problem behaviour revealed that high versus low protection made no difference in the amount of problem behaviour for the no-risk group. However, level of protection did make a significant difference for the moderate and high risk groups; youth with high protection had significantly lower problem behaviour scores than those with low protection and the interaction with risk group was significant. Jessor did not make note of any differences, or lack thereof with regard to gender and protective effects. Historically, the term protective factor has been used primarily within the field of resilience. Thus, for the purposes of the current study, a 
protective factor may be present only if accompanied by moderate to high risk. Furthermore, a protective factor will interact with risk in such a way that the higher the risk, the more buffering a protective factor ought to contribute. Resilience scholars such as Rutter, Giller and Hagel (1998), as well as Masten (2001) also endorse the conceptualization of the protective factor as having a moderating/interacting effect on risk.

\section{Defining a promotive factor}

On the other hand, it may be possible that some positive attributes or strengths will still correlate negatively with undesirable outcomes regardless of the initial level of risk (Farrington, 2003). For example, having a secure attachment and loving relationship with the primary caregiver may prove equally beneficial for both low and high risk youth. This construct will be labelled as a promotive factor-inherently different from a protective factor, given the irrelevance of the initial level of risk (Jones, 2011).

\section{Defining a strength factor}

Finally, the term strength is currently most well-known for its association with the positive psychology movement. According to Tweed, Bhatt, Dooley, and Spindler (2011), the term strength is different from those of resilience or protective factor because it does not imply that the factor is only relevant in the face of risk. Furthermore, Tweed and colleagues (2011) note that where the term strength is used, there is an emphasis on traditional virtues (studied in positive psychology) that often get ignored in other research claiming to identify/explore protective factors. For example, forgiveness, humility, altruism and civic values, gratitude, other esteem, prudence, sense of meaning, and frequent positive affect are all considered virtues (internal strengths) and have received significant attention under positive psychology, but are typically ignored in other research (Tweed et al., 2011). Such strengths are relevant to individual well-being and happiness without a preoccupation with risk situations. For the purposes of this paper, a strength will denote 
any individual, familial, or community characteristic that would intuitively (by face value) seem to be a positive characteristic. Moreover, whether a strength is, or is not, related to recidivism is irrelevant. Comparatively, a protective factor must show a statistical interaction with risk; as one's risk increases, so too does the impact of the protective factor. Finally, to clarify, a promotive factor shows a statistically significant negative correlation with recidivism (i.e., as a promotive factor increases the probability of recidivism decreases); however, the impact of the promotive factor on recidivism remains consistent between low and high risk categories.

\section{The Four Generations of Risk Assessment}

In 1928, Burgess was the first to quantify risk with the development of a measure to assess whether an offender would be a risk for parole. Currently, risk assessment is in its fourth wave and much empirical support has accumulated for the Risk-Need-Responsivity (RNR) principles (Andrews et al., 1990) which constitute the major tenets in correctional risk assessment of the current era. A brief history of the four generations of risk assessment is necessary before detailing the risk-need-responsivity model of offender assessment and rehabilitation.

The first generation of risk assessment was purely based upon the unstructured, professional judgments of offender risk (Bonta \& Andrews, 2007). Risk assessments were performed by probation officers and prison staff as well as psychologists, psychiatrists, and social workers based on their opinions about what risk factors should be considered and how they should be weighted (Bonta \& Andrews, 2007). The early 1970's marked the beginning of the second wave of risk assessment, making use of evidence-based actuarial tools (i.e., measures that used mainly static items that were selected based on their statistical association with recidivism) (Bonta \& Andrews, 2007). At this point, professional judgment had less impact on an offender's risk assessment and the consideration of statistically-determined risk factors took the forefront (Bonta \& Andrews, 2007). Scores on individual, static risk factors (e.g. history of substance abuse) were 
summed such that the higher an offender scored, the greater risk they were at to reoffend (Bonta $\&$ Andrews, 2007). Although lacking in the inclusion of potential treatment targets, the second generation actuarial tools were shown in several reviews to be better predictors of the risk to reoffend than professional judgment (Andrews, Bonta \& Wormith, 2006; Grove, Zald, Lebow, Snitz Nelson, 2000). However, actuarial tools were criticized for relying mainly on atheoretical items of risk simply chosen due to their availability and their correlation with recidivism. Second generation instruments were also critiqued because their "non-criminal history" items still tended to be historical in nature (e.g., history of abuse), thus making it impossible for risk scores to improve after offenders make positive changes in their lives (Bonta \& Andrews, 2007).

The third generation of risk assessment remained evidence-based, but started to incorporate dynamic (current and changing) items in the early 1980s (Bonta \& Wormith, 2007). Criminal history items were still very important, but were combined with dynamic items such as present employment, criminal associates, and status of familial relationships (Bonta \& Andrews, 2007). Evidence shows that these "risk-need" instruments are associated with changes in re-offending (Andrews \& Robinson, 1984; Arnold, 2007; Motiuk, Bonta, \& Andrews, 1990; Raynor, 2007; Raynor, Kynch, Roberts \& Mernington, 2000). The addition of dynamic factors also helps guide staff to make more sound recommendations for interventions geared toward an offender's individual dynamic risks (Bonta \& Andrews, 2007). Most recently, instruments that integrate systematic intervention and monitoring with a more broad range of offender risk factors have been labeled part of the fourth generation of risk assessment (Andrews, Bonta, \& Wormith, 2006). An example of a fourth generation risk assessment tool would be the Level of Service and Case Management Inventory, which incorporates static and dynamic items, a professional override option, and primary needs/intervention targets (Andrews, Bonta \& Wormith, 2004). 


\section{Gender-Responsive Critique of the Risk-Need-Responsivity Model}

The guiding theoretical base for the third and fourth generation risk assessment eras is the risk-need-responsivity (RNR) model of offender assessment and rehabilitation (Bonta \& Andrews, 2007). The risk principle states that the highest intensity of treatment should be reserved for the highest risk cases. The need principle asserts that the act of identifying criminogenic needs serves to create appropriate treatment targets for each unique offender. A criminogenic need is a dynamic (having the capacity to change) risk factor that empirically has been linked to criminal behaviour. According to Andrews and Bonta (2010) there are only seven needs that can be classified as criminogenic. These seven needs are encapsulated within Andrews and Bonta "Central Eight" and include: antisocial personality pattern, pro-criminal attitudes, social supports for crime, substance abuse, family/marital relationships, school/work, and leisure/recreation. The eighth central factor is a static risk factor-history of antisocial behavior. These eight risk factors have been shown to be most associated with recidivism in descending order (Simourd \& Andrews, 1994). Finally, the responsivity principle guides how an offender is to receive treatment. Responsivity means that general cognitive behavioural techniques should be adapted to each offender's individual characteristics (Andrews \& Bonta, 2010). There are two types of responsivity-general and specific (Andrews \& Bonta, 2010). General responsivity refers to the idea that cognitive social learning techniques are the most effective way to teach any kind of new behaviour. Specific responsivity states the consideration of personal strengths and factors such as gender ought to be included in treatment interventions (Andrews \& Bonta, 2010). However, Blanchette and Brown (2006) propose a modification of the responsivity principle where gender and strengths assume a much more central role.

There are two distinct lines of reasoning for how to respond to the female offender population - the "gender neutral" camp and the feminist or gender responsive camp. The gender- 
neutral camp posits that the assessment of female offenders should mirror that of males. In other words, proponents of the gender neutral perspective believe that the RNR model works just as effectively for males as it does for females, regardless of the fact that it was designed for and generally tested on samples of predominately males (Dowden \& Andrews, 1999). Thus, developers of the LSI-R have argued that actuarial instruments are "gender-neutral," meaning they work just as well at assessing female risk as they do assessing risk for males (Rettinger \& Andrews, 2010).

On the other hand, proponents of the gender responsive or feminist camp claim that gender neutral tools neglect factors that are more relevant for female offenders. Furthermore, it has been argued by several feminist-stream corrections researchers that the traditional risk assessment tools in use today are oppressive and do not respect the unique needs of female offenders (HannahMoffat, 2004). Additionally, Holtfreter and Cupp (2007), as well as other feminist stream researchers would argue that the bulk of research in support of the gender neutral perspective uses mostly male samples. Daly's (1992) "pathways to crime" research proposed that there are five categories of female offenders, each following separate trajectories into crime. Thus, feminist scholars contend that measures such as the LSI-R, utilizing the "yes/no" quantifiable format to capture risk, fail to capture the diversity of female circumstances (Holtfreter \& Cupp, 2007).

Some of the life circumstances that are more prevalent among female offenders include complex family backgrounds (e.g., incarcerated women are more likely than incarcerated men to come from a single parent household), backgrounds of physical and sexual victimization, substance abuse, mental illness, never married and/or are a single parent, and have received little education and minimal employment (Bloom et. al, 2005).

In sum, although considered the backbone of risk assessment, the RNR model is essentially deficit-based and there remains much debate as to whether it has been adequately tested with the 
female offender population. In the spirit of positive psychology, correctional researchers are becoming more and more receptive to the idea of incorporating theories of resilience and strengths into the more traditional, deficits-based risk assessment protocols.

\section{Overview of Positive Psychology}

Positive psychology was partially influenced by earlier humanistic perspectives of psychology and has only recently become a recognized sub-discipline of psychology within the last decade (Seligman, 2000). In the words of one of the top proponents of the positive psychology movement, Martin Seligman: "the aim of positive psychology is to catalyze a change in psychology from a preoccupation only with repairing the worst things in life to also building the best qualities in life" (p. 3 as cited in Snyder \& Lopez, 2002). According to Seligman and Csikszentmihalyi (2000), positive psychology is not meant to be a comprehensive perspective upon which to base future work, but as a complementary framework to combine with other psychological perspectives. Although it emerged decades after the first mention of resilience and strength factors in psychology, the discipline of positive psychology can be seen as a broad philosophy to which both of the aforementioned areas apply (Yates \& Masten, 2004). The discipline of positive psychology builds on the pioneering work of Rogers (1951), Maslow (1954, 1962), Jahoda (1958), Erikson (1963, 1982), Vaillant (1977), Deci and Ryan (1985), and Ryff and Singer (1996) to name a few (Seligman, Steen, Park, \& Peterson, 2005).

As mentioned in the previous definitions section, much of the research within positive psychology focuses on "character strengths" or "virtues." In 2004, Peterson and Seligman released the Character Strengths and Virtues: $A$ Handbook and Classification (CSV), meant as a complementary guide to well-being alongside the Diagnostic and Statistical Manual of Mental Disorders (DSM). The CSV relies on six overarching virtues that, according to Seligman and colleagues (2005), almost every culture across the world endorses: wisdom, courage, humanity, 
justice, temperance, and transcendence. The $C S V$ includes chapters specifically focused on what is known and not known about the 24 different strengths of character (Seligman et al., 2005). Each chapter includes paradigm cases, definitions, historical and cross-cultural background, measurement, gender differences, etc...(Seligman et al., 2005). In a cross-cultural study of character strengths (Park, Peterson, \& Seligman, 2005a), the most frequently endorsed strengths by adults around the world were kindness, fairness, authenticity, gratitude, and open-mindedness with correlations between rankings ranging in the $.80 \mathrm{~s}$. Yet, a second study by Park, Peterson, and Seligman (2005b) indicates that the strength profiles of U.S. adults and U.S. youth are more similar than the profiles of U.S. adults and adults from elsewhere around the world. Finally, the strengths of zest, gratitude, hope, and love are more robustly associated with life satisfaction than other strengths among both adults and youth (Park, Peterson, \& Seligman, 2004).

As current research is pointing to gendered pathways to crime (Daly, 1992), positive psychologists are also finding that men and women differ in their experiences and expressions of character strengths. For instance, Kashdan, Mishra, Breen, and Froh (2009) studied the possibility that women, more than men, excel in the experience and expression of gratitude. The results of Kashdan and colleagues' three-part study indicated that men were less likely than women to feel and express gratitude, made more critical evaluations of gratitude, and experienced fewer benefits than women from gratitude.

Although research is beginning to accumulate on the correlations between character strengths and appetitive outcomes, researchers of positive psychology have often neglected to apply positive psychology to more problematic outcomes such as youth violence (Tweed et al., 2011). One area that does typically involve the study of negative life events, including youth violence, is resilience. 


\section{Overview of Resilience Research}

Resilience is a term that has been commonly misused within the literature (Luthar \& Cicchetti, 2000). In their article on resilience and its implications for interventions and social policy, Luthar and Cicchetti (2000) stress the need for researchers to clarify that resilience is not an individual personality trait or attribute. A good way to conceptualize resilience is as an overarching process of overcoming risk (Fergus \& Zimmerman, 2005; Luthar \& Cicchetti, 2000). Thus, a person shall not be labelled as a resilient individual, but one who has experienced a resilient trajectory, or resilient manifestations (Luthar \& Cicchetti, 2000). Resilience is most commonly known as a "dynamic process encompassing positive adaptation within the context of significant adversity" (p. 543, Luthar, Cicchetti \& Becker, 2000). In essence, there are two mandatory components for resilience to take place: 1) exposure to adversity (i.e., negative life circumstances that are statistically associated with adjustment difficulties) and 2) the manifestations of positive outcomes (Luthar \& Cicchetti, 2000). Under resilience theory a positive outcome constitutes "behaviourally manifested social competence, or success at meeting developmental tasks" (Luthar \& Cicchetti, 2000). Furthermore, resilience can be broken down into three sources: 1) attributes of the people themselves, 2) aspects of their families, and 3) characteristics of the wider social environment (Werner \& Smith, 1992). The aforementioned definition of resilience may be appropriate for use with the youth involved in the correctional system as each youth has, at one point or another, experienced an element of risk in his or her life that may, or may not, be followed by resilience trajectories.

Resiliency theory states that the framework applies when there is a positive outcome where high risk exists. Fergus and Zimmerman (2005) would argue that when positive outcomes are present in the context of low risk, this is simply considered normative development. Thus positive outcomes achieved in a situation of low-risk, may be encouraged/reinforced by the earlier 
discussed promotive factors. However, positive outcomes achieved following situations of high risk are reinforced by protective factors/processes resulting in resilient trajectories.

The use of the term resilience first emerged under the study of health sciences and in particular, the study of psychopathology and child development (Rutter, 1979). Among the first scholars to bridge the study of risk and resilience with developmental psychology were Garmezy (1973), Emmy Werner (Werner \& Smith, 1977), and Michael Rutter, 1979. These early pioneers in the area were interested in looking at children's individual responses to familial and environmental stressors and essentially why some children go on to follow a resilient developmental trajectory, while others do not. In addition to making its way into developmental psychology, the study of resilience has since made its way into the field of military psychology, the study of community disasters (Blom, 1982; Johnson, 1980), concentration camp experiences (Langmeirer \& Matejcek, 1975) and more recently, the field of correctional psychology.

One of the earliest and most influential studies of resilience was a multi-wave longitudinal piece by Werner on the children of Kauai, a small Hawaiian community (1982). The Kauai study traced the developmental paths of every child ( 698 participants with a $2 \%$ attrition rate) born in 1955 of a multiracial group of whom approximately $30 \%$ experienced perinatal stress, chronic poverty, and a troubled family environment involving parental psychopathology (Werner, 1982). All of the participants (both males and females) were followed up at ages 1, 2, 10, 18, and 32 years. Analyses included both quantitative and qualitative components.

The children from the 1955 cohort were divided into four groups and each group was also divided by gender. Groups 1 and 2 were comprised of both middle and lower class children, respectively, who had experienced one or more significant stressful life events (e.g. chronic family discord, absence of father, parental mental illness, divorce, death of a sibling, etc.). Groups 3 and 4 were comprised of middle and lower class children, respectively, who had not experienced serious 
or chronic life stressors. Protective factors were essentially operationalized as 1) differentiating high risk children who are resilient from those who develop serious learning and behavioural problems 2) increasing stress resistance 3) important in counterbalancing stress, deprivation, or disadvantage, but that are not important in the absence of such circumstances. Among the nonstressed groups, several variables were identified that discriminated significantly between children who did and did not develop any coping problems: 1) mother's level of education (for males and females), 2) birth order and the number of children in the household (for males), 3) the amount of attention given to the infant during the first year of life (for females), 4) the mother's rating of the infant's activity level (for males), 5) social responsiveness (for females), 6) Cattell IQ at 20 months (males and females), 7) verbal comprehension skills (males), 8) and perceptual motor skills (females). Since these identified variables were identified among low risk groups, they are most easily compared to the definition of promotive factors outlined in the definitions section. Among children who did experience stressful life circumstances in middle or lower class families, or were exposed to deprivation or disadvantage (lower class families), the following variables significantly discriminated between those who experienced good and poor outcomes: good health (males and females), autonomy and self-help skills (for males), positive social orientation and selfconcept (for females), parent-child relationship (both genders), and emotional support from other family members (both genders). These variables are indicative of genuine protective factors as defined earlier in this paper. The classic Werner (1982) piece on the children of Kauai is arguably the most comprehensive study of resilience. The study is longitudinal (ranging a total span of 32 years), looks at both risk and strength factors, and includes results for both genders. However, more recent literature on youth crime usually fails to take such an integrative approach.

\section{Empirical Evidence for Protective and Promotive Factors}

Current literature on crime desistance (i.e., why offenders cease offending) points to both 
internal and external processes as underlying mechanisms of change. As summarized by Serin and Lloyd (2009), external markers (i.e., correlates) of the desistance process commonly include employment (Benda, 2005; Uggen, 1999), abstaining from substance use (Fals-Stewart, 2003; Hussong et al., 2004), marriage (Maume, Ousey \& Beaver, 2005; Sampson \& Laub, 2005) as well as the development of important prosocial relationships (Andrews, 1980). Desistance is also said to be maintained by internal processes such as self-regulation, intrinsic rewards, and long-term goals (Maruna, 2001; Maruna et al., 2004). However, as noted by Serin and Lloyd (2009), the internal processes responsible for juvenile crime desistance may be notably different from those of adults due to their unique life circumstances. Some factors that have been positively correlated with desistance for adults, such as parenthood, may actually be found to be negatively associated with desistance among juveniles (Blokland \& Nieuwbeerta, 2005).

Among the juvenile population, factors such as parenting styles, cognitive skill base, academic achievement, attitudes, mentorship relationships, peer relationships, and extracurricular involvement have been assessed in terms of their protective qualities (Hart, O'Toole, Sharps, \& Shaffer, 2007; Dekovic, 1999, Epstein et al., 2001). Epstein and colleagues (2001) also used the Jessor (1991) framework for understanding protective factors to examine the effects of risk and protective factors on the alcohol use of a sample of seventh grade students. Protective items that were measured included, but were not limited to, "lives in two-parent family," "level of antidrinking attitudes," and "attendance at religious services." For participants at low risk, the cumulative level of protection did not predict alcohol use. However, for participants in the moderate and high risk categories, individuals with higher cumulative protection scores engaged in significantly less alcohol use than those with low protection (Epstein et al., 2001). In support of Jessor's 1991 framework, the entire collection of protective items did moderate outcome (i.e., measures of alcohol use), but the extent to which each protective item contributed to the 
moderated relationship was not reported.

In contrast, other literature finds that protective factors do not have a moderating effect on aversive outcomes. For example, Dekovic (1999) found that the protective effects of family attributes and peer relations are correlated with the development of adolescent problem behaviour, but that when an index of the number of risk and protective factors is used, the amount of risk explained more variation in problem behaviour than the amount of protection. In addition, Dekovic (1999) found that there was no significant moderating effect for the protective factors on problem behaviour. Given that Dekovic did find family attributes and peer relations to be negatively correlated with problem behaviour, it could be that what were assumed to be protective factors, may simply be promotive factors that are beneficial to youth regardless of their level of risk. Although the aforementioned studies shed light on the operation of protective and promotive factors among youth exhibiting problem behaviour, there has not been much specific work referring to youth crime and how protective and promotive factors fit into the assessment of risk among youth in conflict with the law. Furthermore, there has been little attention since Werner's work on the children of Kauai focusing on gender differences and gendered pathways.

Jones (2011) investigated the extent to which promotive and protective domains contribute to risk assessment protocols for young male and female offenders. Jones' (2011) sample was from an archival Orbis Partners database consisting of 1,550 males and 819 females. Significantly more females $(77 \%)$ than males $(57.4 \%)$ were designated as Persons in Need of Supervision (PINS) as opposed to Juvenile Delinquents (JDs). In addition, males included in the sample had more extensive criminal histories than the females in the sample. Using the Youth Assessment and Screening Instrument (YASI), Jones' results indicated that the following domains acted as promotive for females: family, school, peers, attitudes, skills, and free time. Interestingly, none of the identified promotive domains interacted with the overall risk score in the female sample, 
indicating the absence of protective effects. In contrast, the following strength domains were identified as promotive for males: family, school, peers, attitudes, skills, free time, and employment. In addition, both the attitudes and skills domains for males were protective factorsin other words, higher domain scores in attitudes and skills buffered criminal outcome to a greater extent in high risk youth compared to low risk youth. While it is curious that none of the promotive domains proved to be protective in the female sample, at the very least, Jones' results indicate the need to properly distinguish between protective and promotive factors. Also note that the lack of female specific findings may have been more of a statistical artifact given that the majority of females in the sample were low risk. In addition, a "gender-neutral" version of the YASI was being used, which could have possibly biased the results. Even still, Jones' results do support the need for adopting a gender-informed as well as a strength-based approach to risk assessment given that genuine protective effects were found for males in her sample. Two current risk assessment tools for youth that have made the point of trying to capture strengths are the Youth Level of Service and Case Management Inventory (YLS/CMI; Hoge \& Andrews, 2010) and the Youth Assessment and Screening Inventory (YASI) (Orbis Partners, 2007). However, both tools utilize different methods of including strength and/or protective factors.

\section{Youth Level of Service and Case Management Inventory}

The Youth Level of Service and Case Management Inventory 2.0 (YLS/CMI 2.0) (Hoge \& Andrews, 2010) is grounded in the General Personality and Social Psychological Model of Criminal Conduct (Andrews \& Bonta, 2006; Andrews, Bonta \& Hoge, 1990). The tool was designed to assist correctional workers in making reliable and valid decisions about adolescent offenders in terms of the identification of risk and need factors (Hoge \& Andrews, 2010). It is comprised of eight domains-offense history, family circumstances/parenting, education, peer relations, substance abuse, leisure/recreation, personality/behaviour, and attitudes orientation. In 
addition to assessing areas of risk and need for youth, the YLS/CMI 2.0 also seeks to address offender responsivity factors. To reiterate, responsivity factors are not necessarily associated with criminal activity, but are nonetheless important in the way a youth reacts to different types of interventions (Hoge \& Andrews, 2010). Since the responsivity principle suggests that strengths should be taken into account when considering the most appropriate intervention, Hoge and Andrews (2011) include a "strength" category in each assessed risk/need domain of the tool. The assessor simply checks off "strength" if deemed applicable. However, during their discussion about including strengths, Hoge and Andrews (2010) use the term protective factors interchangeably with strength factors-two terms which, as already noted, are used for two different constructs. The YLS/CMI 2.0 manual gives no further direction to clinicians on how to recognize "strengths" and how they can be included into the overall assessment of risk as so-called moderators of the impact of risk factors. If a clinician believes that a youth possesses a strength in a certain domain, then they may check off the strength box. In some particular domains (e.g. criminal attitudes) a youth cannot possess both a strength and risk according to the YLS/CMI 2.0 manual. To date, there has not been any published research done on the strength components of the YLS/CMI 2.0.

\section{Youth Assessment and Screening Instrument}

The Youth Assessment and Screening Instrument (YASI) is a tool developed by a Canadian consulting firm, Orbis Partners (2000) designed for use in juvenile probation and other high-risk youth service settings. The development of the YASI was heavily influenced by the four principles of effective case management outlined by Andrews and colleagues (risk, need, responsivity, and program integrity). Similar to the YLS/CMI, the YASI assesses "strengths" in seven of its ten domains: family, school, community/peer, employment, use of free time, attitudes, and skills. However, in contrast to the YLS/CMI 2.0, the YASI measures each strength on a 
numerical scale (a continuum) that ranges from $(-2)$ (i.e., does not possess the strength) to $(+2)$ (i.e., very much possesses the strength). Each youth is given a protective score for each of the seven domains in addition to an overall protective score. Please note that although the YASI manual uses the term, "protective factors", in practice, they should actually be called promotive factors given the lack of current evidence that YASI promotive domains add incrementally to the prediction of recidivism. Therefore, in this section a "YASI protective factor" is, in reality, a promotive factor. A youth can score either "zero, low, moderate, or high" protection within each domain and then again for an overall promotive score using the algorithms designated by Orbis Partners in the Scoring Manual for the YASI (see methods section for further discussion). Therefore, the YASI uses a pre-specified statistical algorithm for identifying protective factors than the YLS/CMI, however both tools utilize protective factors strictly for case management targets and noted protection does not play a role in a youth's overall calculation of risk for either tool.

Contrary to the YLS/CMI 2.0, there have been two studies in particular that assess the protective component of the YASI. A two year fixed follow-up study involving New York State Juvenile Probation youth (Orbis Partners, 2007) found that when using the 6-level overall protective scores, based on the summation of all protective factor items in the YASI battery, most AUCs hovered around the 0.59 level, suggesting satisfactory prediction of recidivism and poorer performance than dynamic risk scores at predicting outcome (AUCs hovered around .63 for dynamic risk scores). An examination of the linear trends between the YASI protective factor categories (i.e., zero low, moderate, and high) suggested inverse relationships between protective factors and recidivism, but the existing cut-offs only provided good discrimination at the extremes-i.e., low and very high. In addition, there was little difference in the AUCs for males and females. However, there was a tendency to classify females in the low protective category. 
In terms of the outcome by individual protective factor domains, AUCs ranged from 0.50 to 0.58 .

Orbis Partners (2007) also investigated the interaction between risk and protective factors in their long-term validation study. Orbis Partners (2007) assert that protective factors are not simply the reverse of risk. In line with this theory, protective factors are separate constructs that buffer/reduce the effects of risk and should add additional predictive power to the tool (Orbis Partners, 2007). Results indicated that the "buffering hypothesis" was relevant for high risk juvenile delinquents and was found to work similarly for both males and females although evidence was fairly weak suggesting the possible need to reweight some items.

Recalling Jones' (2011) findings, evidence was found that family, school, peers, attitudes, skills, and free time domains all acted as promotive for females (i.e., were negatively associated with outcome); however, in contrast to Orbis Partners (2007), none of the domains were found to be genuinely protective for females. Furthermore, family, school, peers, attitudes, skills, free time, and employment were found to be promotive for males and only attitudes and skills were genuinely protective for males.

Further research on samples of youth who have been assessed by both the YASI and YLS/CMI 2.0 is needed in order to determine more clear relationships between the strength/protective components of each tool and the youth's level of risk and on their eventual outcomes.

\section{Current Study}

In sum, justice-involved youth are a unique and vulnerable group that require special consideration, different sanctions, and tailored interventions. Strengths-based perspectives such as the Good Lives Model for offenders have yet to be adapted for justice involved youth. In addition, feminist corrections researchers advocate strength-based and empowerment-based platforms for the treatment of female offenders, yet this area is still understudied. Not only is evidence mixed for 
the methods of assessment of risk in justice involved females, but there is even less research on gender differences and strengths/promotive/protective factors.

Risk assessment tools and protocols have traditionally failed to capture the strengths of individual youth and further, do not provide guidance on how strengths-based perspectives fit in to risk assessment. While methods of risk assessment have been well-established, methods for assessing promotive and protective factors among youth in conflict with the law have not. Guided mainly by resilience theory and in an attempt to fill the gaps in existing literature, the current study aims to do the following:

Research objective \#1: determine if the number and type of strengths as measured on the YLS/CMI 2.0 and YASI respectively vary as a function of gender.

Hypothesis \#1: to determine if the number and type of strengths as measured on the YLS/CMI 2.0and YASI vary as a function of gender, an exploratory approach will be used due to the lack of previous research on the strength components of the YLS/CMI and lack of research on the YASI tool.

Research objective \#2: Determine how well the YASI and YLS/CMI 2.0 strengths/protective factors - both at a composite level and individual domain level-correlate with each other (where comparable) for males and females separately.

Hypothesis \#2: Since both the YLS/CMI 2.0 and YASI manuals make reference to measuring "protective factors," it is hypothesized that composite strength/protective scores on the YLS/CMI 2.0 will be positively correlated with the comparable YASI strength/protective scores, as well as domain level strength/protective scores where comparable. There is no hypothesis for differences that may or may not be found between males and females.

Research objective \#3: establish if YLS/CMI 2.0 identified strengths add incrementally to the post-diction of criminal history above and beyond risk (inclusive of criminogenic need factors) 
and whether or not this relationship is moderated by gender.

Hypothesis \#3: to establish if YLS/CMI 2.0 identified strengths add incrementally to the post-diction of criminal history above and beyond risk and whether or not this relationship is moderated by gender an exploratory approach will be utilized due to the scarcity of existing research.

Research objective \#4: establish if YASI identified protective factors add incrementally to the post-diction of criminal history above and beyond risk, as well as whether or not the relationship is moderated by gender.

Hypothesis \#4: to establish if YASI identified protective factors add incrementally to the post-diction of criminal history above and beyond risk, as well as whether or not the relationship is moderated by gender an exploratory approach will be used due to the scarcity of existing research.

\section{Method}

\section{Participants}

Participant data being used for this project is archival and was collected as part of a larger study entitled "Gendered Pathways to Crime: Implications for Risk Assessment." Data was collected at several sites. Data for this project is based on 120 participants for whom data had been collected as of January 2012 (59 female justice involved youth; 61 male justice involved youth). The participant data being used in the current study comes from justice-involved youth who were assessed while in closed custody, open custody or probation. More specifically, youth were assessed at one of the following sites: Roy McMurtry Youth Centre (RMYC; closed custody facility for male and female youth, Brampton, Ontario), William E. Hay Youth Centre (WHYC; closed custody facility for male youth only, Ottawa), Elizabeth Fry Attendance Centre (a probation attendance/programming centre for female youth, Brampton, Ontario), Marjorie Amos Residence (an open custody facility for female youth, Brampton, Ontario), Sherwood House (an open custody 
facility for female youth, Ottawa), and Ottawa Probation. A coding manual was completed for each youth at the time of interview documenting demographic information including age, gender, ethnicity, location of interview, current and aggregate dispositions, current, and aggregate offence details, and official convictions in criminal history based on the interview and systematic file review of each youth (See Table 1 for sample descriptives). Note that only three 14 year old youth were included in the sample, along with two 20 year olds serving a youth sentence. For ease of interpretation, current and aggregate offences were broken down into violent and non-violent (See Table 2).

Table 1

Sample Descriptives: Demographic Information

\begin{tabular}{|c|c|c|c|c|}
\hline \multirow[b]{2}{*}{ Characteristic } & \multicolumn{2}{|c|}{ Male $(N=61)$} & \multicolumn{2}{|c|}{ Female $(N=59)$} \\
\hline & $n$ & $\%$ & $n$ & $\%$ \\
\hline \multicolumn{5}{|l|}{ Age } \\
\hline $14-16$ years & 21 & 34.4 & 24 & 40.7 \\
\hline $17-20$ years & 40 & 65.5 & 35 & 59.3 \\
\hline \multicolumn{5}{|l|}{ Ethnicity } \\
\hline African American & 16 & 26.2 & 13 & 22 \\
\hline Caucasian & 30 & 49.1 & 40 & 67.7 \\
\hline Aboriginal & 3 & 4.9 & 2 & 3.4 \\
\hline Other & 12 & 19.7 & 4 & 6.7 \\
\hline \multicolumn{5}{|l|}{ Disposition } \\
\hline Remand & 25 & 40.9 & 35 & 59.3 \\
\hline Custodial sentence & 19 & 31.1 & 12 & 20.3 \\
\hline Community Sentence & 13 & 21.3 & 11 & 18.6 \\
\hline Other & 4 & 6.5 & -- & -- \\
\hline
\end{tabular}

Note. Other ethnicity includes: biracial ethnicity, Jamaican, Asian, Hispanic, European, South Pacific, and Middle Eastern ethnicities. Other disposition includes: Community service hours and Intensive Rehabilitative Custody and Supervision Order (IRCS). 
Table 2

Violent and Non-Violent Offence Classification: Current Index, Aggregate, and Past Criminal Offence Information

\begin{tabular}{llllrr} 
& \multicolumn{2}{c}{ Male $(N=61)$} & \multicolumn{2}{c}{ Female $(N=59)$} \\
Offence Type & $n$ & $\%$ & $n$ & $\%$ & $X^{2}$ \\
\cline { 2 - 6 } & 46 & 75.4 & 33 & 55.9 & 2.738 \\
\hline Violent & 53 & 86.8 & 55 & 93.2 & .446 \\
\hline
\end{tabular}

Note. Violent offences include: homicide, robbery, major assault, and sexual offences. Non-violent offences include: theft, break and enter, narcotics, minor assault, driving offences, weapons, threats, breaches, prostitution, and fraud.

In order to control for confounding variables in analyses, equalizing the sample in terms of offence types was important. Potential gender differences in the combination of criminal history offences, aggregate admitting offences, and current offences (i.e. offences the youth has been convicted of and is serving a sentence for) were examined in the following way: originally, field researchers used official files to code whether or not a youth had been charged and/or convicted with one of the following 21 offence types: theft, break and enter, narcotics (using), narcotics (selling/transporting), robbery (no weapon), robbery (with weapon), minor assault, major assault, homicide, serious driving, weapons possession, weapons use, threats, breaches, unlawfully at large, escapes, sexual offences, prostitution, frauds, obstruction of justice, and other. For ease of interpretation, the following offence categories were amalgamated: theft and property offences; both narcotics offences (possession and trafficking) were joined into one variable called drug offences; both robbery offences were collapsed into one variable; breaches, unlawfully at large, and escapes were all combined into one variable; and weapons possession and weapons use were combined resulting in 15 offence types. 15 chi-square analyses were conducted to ascertain whether differences existed for each offence type by gender. Chi-square results indicated that there were five distinct variables that showed gender differences where $p<.05$ : theft and property 
offences, drugs, robbery, and weapons offences. Notably, there were clear gender differences for sexual assault and homicide with males accounting for either all or disproportionately more of these crimes, however analyses could not be done due to the violation of a two by two chi-square expected cell count of at least five. The remainder of the chosen gender distinct offences had substantially higher chi-square values than those offences that did not meet inclusion except for the prostitution offences and the sexual offences that did not meet inclusion because they had no male or female counterparts (See Table 3).

Table 3

Nature of Offence Type by Gender.

\begin{tabular}{lccc}
\hline Offence Type & Males & Females & $X^{2}$ \\
& $\%(74)$ & $\%(73)$ & \\
\hline Robbery & $63.5(47)$ & $20.5(15)$ & $28.423^{* * *}$ \\
Weapons & $48.6(36)$ & $15.1(11)$ & $19.486^{* * *}$ \\
Theft \& Property & $75.7(56)$ & $47.9(35)$ & $12.583^{* * *}$ \\
Sexual & $10.8(8)$ & -- & - \\
Drugs & $35.1(26)$ & $15.1(11)$ & $7.856^{* *}$ \\
Homicide & $9.5(7)$ & $2.7(2)$ & -- \\
Breaches & $87.8(65)$ & $79.4(58)$ & 2.358 \\
Other & $71.6(53)$ & $63(46)$ & 1.348 \\
Prostitution &.- & $1.4(1)$ & - \\
Serious Driving & $5.4(4)$ & $2.7(2)$ & .724 \\
\hline
\end{tabular}

Note. ${ }^{* * *}=p<.001,{ }^{* *}=p<.05,{ }^{*}=p<.10$. Chi-square analyses could not be conducted for sexual, homicide, prostitution, or fraud offences due to expected cell count less than 5 . 
Table 3 Continued

\begin{tabular}{lccc}
\hline Offence Type & Males & Females & $X^{2}$ \\
& $\%(74)$ & $\%(73)$ & \\
\hline Fraud & $2.7(2)$ & $5.5(4)$ & -- \\
Minor Assault & $39.2(29)$ & $46.6(34)$ & .691 \\
Major Assault & $45.9(34)$ & $39.7(29)$ & .691 \\
Threats & $28.4(21)$ & $32.9(24)$ & .350 \\
Obstruct Justice & $13.5(10)$ & $13.7(10)$ & .000 \\
\hline
\end{tabular}

Note. ${ }^{* * *}=p<.001,{ }^{* *}=p<.05,{ }^{*}=p<.10$. Chi-square analyses could not be conducted for sexual, homicide, prostitution, or fraud offences due to expected cell count less than 5 .

Given these results an effort was made to match the males and females on offence type using a matching technique. This was accomplished in two steps. First, a new 'gender distinct offence' variable was created by summing the six variables that evidenced significant (i.e., robbery, weapons, theft and property, and drug offences) or meaningful (sexual and homicide offences) gender differences. Each variable was assigned either a 0 offence type not present, $1-$ offence type present. Thus, total scores could range from 0 to 6 . Next, male scores on the gender distinct offence variable were matched with female scores on the variable. The matching was executed in a way that all of the possible female sample was used. For instance, if 5 females all scored a 1 on the gender distinct offence variable, 5 males also scoring a 1 would be chosen at random (using the SPSS random sample function) to be included with the 5 females in the final data set. The data set with matching by the gender distinct offence variable resulted in 120 cases (59 females; 61 males) ${ }^{1}$. Perfect matching could not be achieved due to several female cases that required deletion during the data cleaning process. An independent samples t-test indicated that

\footnotetext{
${ }^{1}$ The sample size was too small to permit a fully matched blocked procedure by offence history and racethus, blocked matching was used for offence history, but not race
} 
females $(M=1.25, S D=.86)$ did not significantly differ from males $(M=1.13, S D=.88)$ on the gender distinct offence variable in the matched data set, $t(120)=(-.771), p=.442$.

\section{Procedure}

As stated, the proposed study is archival in nature. Regardless, a brief synopsis of the original data collection procedures of the project will follow. Seven research assistants (graduate level psychology students and two recently graduated honour's psychology students) were trained to administer both the YASI and YLS/CMI 2.0. The developers of the YASI as well as the YLS/CMI 2.0 were recruited to provide training to the research assistants (including me) on how to use their tools. Approval from the Ministry of Children and Youth Services and the Carleton University Ethics Board was gained before participant recruitment began. A court order was also secured to conduct the research.

Once researchers were fully trained on administering the assessment tools, a list of all possible candidates for the study was provided by the institutions on an on-going basis. Adolescent offenders were approached individually by the research assistants and the study was subsequently explained and broken down in a way that was easily understood by the participants. Interested youth aged 16 years or older were provided with an informed consent form on the spot. Youth under the age of 16 needed a parent/guardian or a Children's Aid Society case worker to complete a parental consent form before they were allowed to participate. After consent was gained, the research assistant first conducted a review of the particular youth's file looking for current, aggregate, and historical offence information. Research assistants also took note of any other reports or incidents noted in the files.

Once the file review was complete, the research assistant conducted a two to three hour, tape recorded (with consent) interview with the youth, asking questions pertinent to rating items on the YLS/CMI 2.0, YASI, and the Psychopathy ChecklistYouth Version (PCL-YV). 
The interviews were conducted in a private office space in the relevant institution, or in the probation office or in a quiet public area if sufficient privacy was possible. The first part of the interview was spent trying to get as much detailed information about the current offense as well as past offence history as possible in order to provide a detailed narrative that aided in the scoring of the risk assessment measures. The second part of the interview was spent getting information about the youth's family life, mental health difficulties, school, and so forth. Participants then filled out a short battery of questionnaires. Participants received $\$ 30.00$ for their participation that was either credited to their institution canteens (at RMYC), or distributed in Tim Hortons, Walmart, or Chapters gift cards (all other participating sites opted for this option).

At the conclusion of the interview, youth were debriefed regarding the importance of the study and whom they should talk to in the event of adverse emotions resulting directly from the study. Time was also taken at the end of the interview to make sure that the youth ended the interview in the same state of mind they began with (i.e., they were not in any more psychological distress than they started with). Thus, we often ended the interview with a discussion of the youth's strengths. If the youth had spoken about upsetting issues during the interview process, they were given the option of speaking to a psychologist afterward.

Once the research assistant had completed the first three components (file review, interview, and questionnaires), they completed the YLS/CMI 2.0, YASI and PCL-YV assessments. Consent forms and other identifying documents were kept separately from interview notes and risk assessment measures. Inter-rater reliability was assessed for the YASI and YLS/CMI 2.0 based on 20 cases ( 10 males and 10 females) that were randomly selected from the entire sample.

\section{Materials}

YLS/CMI 2.0. The Youth Level of Service and Case Management Inventory 2.0 (YLS/CMI 2.0) 
(Hoge \& Andrews, 2011) stems from the Level of Supervision Inventory (LSI: Andrews, 1982) the original adult version of the measure which was then modified into the Level of Supervision Inventory-Revised (Andrews \& Bonta, 1995), and finally, the Youth Level of Service Inventory and Case Management Index (Hoge \& Andrews, 2002) before arriving at the YLS/CMI 2.0. The measure includes eight subscales: offense history, family circumstances/parenting, education, peer relations, substance abuse, leisure/recreation, personality/behaviour, and attitudes orientation. Each subscale includes three to seven items (42 in total) rated as yes or no. Risk/need items are then summed to provide an overall score ranging from 0 to 42 . Each subscale (excluding criminal history) also includes one check box to indicate possible strengths in any of the domains. Depending upon a youth's total score, his/her gender, and custody placement (i.e., custody or community) he/she will be classified into one of four overall risk levels: low, moderate, high, or very high. Currently, strengths are not incorporated into the overall risk score. In addition, the primary use of the strengths check boxes on the YLS/CMI 2.0 is for case management. Youth are not designated into low, high, and moderate categories according to their strengths.

YLS/CMI 2.0 Psychometric Properties. The internal consistency of the YLS/CMI 2.0 has been studied in approximately eight studies that were mentioned in the scoring manual (Hoge \& Andrews, 2011). Chronbach alphas range from .64 to .86 for males and .61 to .75 for females on each subcomponent of the YLS/CMI 2.0 and from .73 to .90 for the total risk/need score indicating good internal consistency (Hoge \& Andrews, 2010; Flores, Travis \& Latessa, 2004; Kimonis et al., 2008; Marshall et al., 2006; Welsh et al., 2008). Of five studies that report interrater reliability for the YLS/CMI 2.0, intraclass correlations (ICCs) for the total score ranged from .72 to .98 (Catchpole \& Gretton, 2003; Marczyk et al., 2005; Viera, Skilling, \& Peterson-Badali, 2009; Viljoen et al., 2009; Welsh et al., 2008). ICCs for the subcomponents of the YLS/CMI 2.0 ranged from .05 to .92 (Welsh et al., 2008). Hoge and Andrews (2011) reported that the validity of the 
YLS/CMI 2.0 was assessed by comparing mean scores between the community and custodial normative samples for males and females (scores were expected to be higher on average for custodial offenders than community offenders). ANCOVA results for males demonstrated significantly higher mean YLS/CMI 2.0 total scores for the custodial sample than the community sample $(d=1.03)$. In addition, higher mean YLS/CMI 2.0 total scores were found for the female custodial sample compared to the female community sample $(d=1.32)$. Hoge and Andrews (2011) also reported large differences for the subcomponents of the YLS/CMI $2.0(d=0.43$ to $d=1.42$ for males) ( $d=0.59$ to $d=1.47$ for females). Such results have been replicated in approximately 4 studies with effect sizes ranging from .05 to 1.27 for subcomponent scores (Marczyk et al., 2005) and effect sizes ranging from .58 to 1.41 for total scores (Bechte, Lowenkamp, \& Latesssa, 2007; Marshall et al., 2006; Marczyk et al., 2005). Hoge and Andrews (2006) as well as others have reported good predictive validity of the YLS/CMI 2.0. YLS/CMI 2.0 total and subcomponent scores were significantly correlated with official records of general recidivism among youth. Reports of the predictive validity of the YLS/CMI 2.0 have been summarized in a variety of metaanalyses. Edens, Campbell and Weir (2007) found significant weighted mean correlations for the YLS/CMI 2.0 in predicting general recidivism and violent recidivism. Schwalbe (2007) also reported a weighted mean AUC of $.64(p<.05 ; \mathrm{k}=11, n=3,265)$ indicating that overall, a recidivist will show a higher YLS/CMI 2.0 score than a non-recidivist (Hoge \& Andrews, 2011 ). In terms of gender differences (Schwalbe, 2008a) also report that the YLS/CMI 2.0 prediction of re-offense was not statistically different for males $(r=.32)$ and females $(r=.40)$.

YASI. The YASI is a comprehensive risk/need/protective factor assessment tool designed for youth in probation services and other high-risk youth service settings (Orbis Partners, 2007). The YASI is primarily grounded in the gender-neutral literature, and its foundation was largely based on male samples (Orbis Partners, 2007). However, most recently Orbis Partners have 
introduced a modified tool (YASI-G) that includes the addition of gender-responsive items to improve its predictive validity with female samples. The development of the YASI was based on the Case Management Assessment Protocol (CMAP; Barnoski, 2003), a similar assessment tool developed for Washington State juveniles. The CMAP assessment tool was selected as a model for the YASI in particular due to its inclusion of strength based factors (Orbis, 2007a). The development of the YASI differs from other assessment tools in that 1) consultation with frontline workers involved in the delivery of probation services aided in the development of certain items, response styles, and the order of items 2) additional items were added to enhance understanding of a youth's strengths; 3 ) items were added to reflect mental and physical health issues; and 4) certain items' weights were adjusted to better reflect the dynamic predictive validity of the measure (Jones, 2011).

The YASI is widely applied across New York State, but has also been implemented across Illinois, North Dakota, Vermont, Virginia, Mississippi, and California (Orbis Partners, 2007a). More recently the YASI has been utilized with youth populations in Scotland, and Alberta, Canada (Orbis Partners, 2011). Each Jurisdiction the YASI is implemented in uses a modified version of the measure that has been specifically designed in collaboration with various agencies of relevance to that jurisdiction (Orbis Partners, 2007).

This study used a modified version of the YASI (Alberta version) and YASI-G (a newly developed gender responsive version of the tool currently not used in pratice). More specifically, the tool was modified so that each youth was asked all of the original YASI questions and all of the gender responsive questions unless the question clearly did not apply (e.g. a male would not be rated as having pregnancy related health issues). The scoring of the YASI relies on an in-depth interview and collateral file review, police reports, school records, mental health reports, and so forth. However, all of the scoring algorithms used to interpret youth responses statistically were 
based on the Alberta version of the YASI. Currently, Orbis Partners has not released a specific set of scoring algorithms for the YASI-G.

YASI Pre-Screen. The YASI pre-screen, comprised of a subset of items from the full assessment, is primarily used for triage purposes at the intake stage of probationary services. The pre-screen developed for Alberta uses 31 items taken from the legal history and social domains of the full assessment. Please consult Appendix A for a detailed breakdown of the pre-screen items as well as the associated cut-off scores. The pre-screen is comprised of both static and dynamic risk items and is, for the most part, scored on likert-type scales resulting in 5 response options. In addition, a variety of items are scored simply using raw scores (e.g. number of times placed on remand). Having a variety of response options helps to increase the sensitivity of the tool and also aids in its ability to capture change at the item level (Orbis Partners, 2007a). The scoring of the pre-screen requires that the items be summed into a total pre-screen risk score and from there, a youth will be classified as low, moderate, or high risk in accordance with the risk principle. The weighting of items and procedures for developing cut-offs is described in more detail below.

YASI Full Assessment. The YASI full assessment consists of 10 subscales: legal history, family, social networks, mental health, substance use, school, attitudes, skills, employment $\&$ free time, and violence/aggression and 113 individual items. Consult Appendix $\mathrm{C}$ for the full break down of items by sub-domain. Each item is scored on a 5-point scale ranging from (-2) to 2 , where a (-2) denotes that a particular item is a risk item for the youth and a 2 denotes that the item acts as protection for the youth. The YASI full assessment is comprised of both static and dynamic risk and protective items. To recap, when an item is static, the rater takes into account all previous and current behaviour/circumstances, whereas if the item is dynamic the rater takes into account only behaviour/circumstances in the last three months.

Orbis Partners used a statistical weighting procedure for each YASI item based on the 
item's association with recidivism; the actual weighting procedure was not specified, but it is understood that the developers of the YASI used a method of scoring items that was loosely based on Nuffeild (1982) whereby item levels were weighted in accordance with recidivism base rates. Relevant items are then summed to yield the following four ratings: total static risk, total dynamic risk, total static protective, and total dynamic protective. Also, note that YASI items and the cutoffs for each sub-domain are jurisdiction specific. Cutoffs were developed by looking at the frequency distribution for the range of scores on either the subdomain or total scores, as well as the distribution of recidivism rates associated with those scores. The recidivism base rate was ideally captured in the average for the medium risk category. The developers essentially looked for natural jumps in the distributions, making sure that there was a sufficient increase in the average recidivism rate associated with adjacent groups and an adequate number of subjects in each group to determine cutoffs (low, moderate, and high) (N. Jones, personal communication, May, 2012). In addition, the YASI also generates the same four ratings (static risk, dynamic risk, static protective, and dynamic protective) at the sub-domain level where relevant, as well as applies cut-offs at the subdomain level (see Appendix B). In practice, only the quantitative score from the pre-screen is used for youth classification purposes (i.e. low, moderate, high). The full assessment does generate quantitative scores and classifications, but they are used to guide intervention efforts and case management (Jones, 2011).

Psychometric Properties of the YASI. The YASI was originally validated in 2007 (Orbis Partners, 2007) on a sample of 3,263 justice involved youth probationers in New York State. The sample was comprised of $70 \%$ Caucasian youth, $18.9 \%$ African American youth, and approximately $11 \%$ other ethnicities. Males represented $65.2 \%$ of the sample while females represented $34.8 \%$ of the sample. Twenty-one percent of the sample was categorized as very high risk on the YASI, while the rest of the sample was equally divided among the low, low-moderate, 
moderate, and moderate-high groups.

Jones (2011) reported the internal consistency of the YASI using Cronbach's alpha on a sample of 819 females and 1,550 males from the New York State probation division. The YASI pre-screen generated Cronbach's aphas ranging from .54 to. 65 , noting that the diversity of items included in the pre-screen are likely the cause of the low internal consistency. Alpha ranged from .42 to .85 for females on the YASI full-assessment risk domains and from .42 to .86 for males. In addition, alpha ranged from .45 to .91 in the strength domains of the YASI full-assessment for females and from .47 to .92 for males (Jones, 2011). The YASI full-assessment domains that evidenced acceptable reliability with Cronbach's alpha levels exceeding .70 include skills, attitudes, employment (strength component), and peers and community (risk component) (Jones, 2011).

In partnership with Orbis Partners and the University of California, the California Department of Juvenile Justice (DJJ) examined the inter-rater reliability of the California version of the YASI (Skeem et al., 2012). The extent to which DJJ staff were able to reliably score the YASI based on six videotaped interviews and corresponding file information was examined. DJJ staff ratings were compared to the ratings determined by YASI expert consensus. Intra-class Correlation Coefficiencts (ICCs) indicated that $60 \%$ of the $78 \mathrm{DJJ}$ staff demonstrated at least adequately reliable YASI total scores $(M \mathrm{ICC}=.63, S D=.36)$. However, average levels of reliability were adequate at each site for only about half of the YASI sub-domains with average ICC's ranging from .11 (social cognition domain) to 1 (education/employment domain). The report concluded that most sites did not attain adequate reliability on scales that theoretically tap into major risk factors associated with antisocial attitudes, criminal thinking, and criminogenic peer relationships (Skeem et al., 2012). 
AUC's for the YASI pre-screen risk ranged from .56 to .67 for females and .58 and .68 for males for predictive outcomes within a 12 month period of being scored. In other words, scores on the YASI predicted negative outcomes at a higher rate than chance. Orbis Partners (2007) note in their results that females generally had lower failure rates than males in both 12 month and 24 month fixed follow-up periods. The data suggested that girls were being over-classified in the high risk category, suggesting the need for the different scoring cut-offs based on gender that can be found on the Alberta version of the YASI measure.

Although not part of risk classification in practice, the YASI also quantitatively measures a youth's protective qualities. The 2007 validation study of the YASI includes some psychometric data on these protective qualities that are important to mention given the scope of the current research. A large number of youth in the 2007 validation study, $32 \%$, were scored in the low protective factors category, while the rest were equally divided among the remaining protective categories (i.e. moderate and high). The data suggests a resilience effect for high-risk cases that exhibited high protective factors. The outcomes show that high risk juvenile probationers with a high level of protective factors (strengths) had outcomes that were more comparable to low risk offenders than the high risk youth who had low protective factors. In terms of the outcome by individual protective factor domains, AUCs ranged from 0.50 to 0.58 , suggesting that they do not predict outcome better than chance alone.

\section{Results}

\section{Data Screening}

Missing data. All data was screened for data entry errors and missing data. Data entry errors were corrected prior to running missing value analyses. Two cases were deleted due to extreme amounts of missing information (both were pilot cases). A missing value analysis (MVA) revealed that the percentage of missing data for the eight YLS/CMI 2.0 subdomain scores (e.g. 
substance abuse) was minimal ranging from $1.6 \%$ (i.e., employment, peers, leisure, personality, and attitudes subdomains) to $4.9 \%$ (i.e., family subdomain) for males. The percentage of missing data for females ranged from $4.9 \%$ (i.e., criminal history, peers, substance abuse, leisure, personality, and attitudes subdomains) to $11.5 \%$ (i.e., family subdomain). The pattern of missing data was assessed using Little's missing completely at random (MCAR) test. The missing data for females was found to be missing at random, $X^{2}=8.78(14), p=.845$ (i.e., the pattern of misingness on any given subdomain was not related to the remaining subdomains collectively). Similarly, the pattern of missing data was found to missing at random for males, $X^{2}=25.33(21), p$ $=.233$. The $t$-tests generated by the MVA analyses indicated that the patterns of missingness differed between males and females on only two of the variables (i.e., the education variable and the peers variable). Specifically, it appeared that the YLS/CMI 2.0 total score was significantly related to missing data on the YLS/CMI 2.0 education variable for males $t(4.8)=2.8, p=.041$, but not for females. The second difference in missingness was on the YLS/CMI 2.0 peers variable where females showed a significant relationship between the YLS/CMI 2.0 score and the YLS/CMI 2.0 peers variable $t(8.5)=-2.9, p=.018$, but males did not.

An additional MVA revealed that the percentage of missing data for the $28 \mathrm{YASI}$ subdomain scores was also minimal ranging from $1.6 \%$ (i.e., legal history, family, substance abuse, and mental health sub-domains) to $11.5 \%$ (i.e, education sub-domain) for males. Similarly, missing data on YASI sub-domain scores for females was minimal ranging from $1.6 \%$ (i.e., family dynamic risk, education, aggression, attitudes, skills, and employment sub-domains) to $11.5 \%$ (i.e., family static risk subdomain). The pattern of missing data was assessed using Little's MCAR test. The missing data for females was found to be missing at random due to non-significant results on the Little's MCAR test, $X^{2}=93.35(186), p=1.00$. Similarly, the pattern of missing data for males was found to be non-significant using Little's MCAR test, $X^{2}=14.206(128), p=.097$. In other 
words, the pattern of missingness on YASI sub-domain scores was not related to the other subdomain scores as a whole. However, the pattern of missingness was different between males and females in some cases. The t-tests generated by the MVA analysis for the YASI sub-domain and total scores indicated that patterns of missing values differed on seven YASI variables between males and females. Specifically, it appeared that the YASI education static risk score was significantly related to missing data on the YASI Legal static risk variable for females $t(55.7)=$ $2.8, p=.007$, but not for males. Another difference in missingness was on the YASI education static risk variable where females showed a significant relationship between it and the YASI family dynamic protective variable $t(55.1)=2.1, p=.039$, but males did not. The YASI education static risk variable was also significantly related to missing data on the YASI employment static protective variable for females $t(3.0)=3.5, p=.038$, but not for males. Missing data on the YASI education static risk and employment dynamic protective domains were also significantly related for females $t(3.0)=-3.8, p=.031$. Missing data on the YASI substance abuse dynamic risk variable and legal static risk variable was significantly related for females $t(53.3)=3.1, p=.0031$, but not for males. Missing data on the YASI substance abuse dynamic risk variable was significantly related to the missing data on the YASI family static risk variable for females $t(49)=$ $2.1, p=.043$, but not for males. Finally, missing data on the YASI substance abuse dynamic risk variable and the YASI family dynamic protective variable was significantly related for females $t$ $(53.1)=2.0, p=.047$, but not for males.

Thus, missing data was minimal for both the YLS/CMI 2.0 and the YASI. However, after closer inspection of the results, based on an unmodified dataset (i.e., missing values were not dealt with), it was discovered that the use of listwise deletion would result in a 20 to 35 percent loss in participant data. Therefore, a method of multiple imputation (MI) was adopted, which is an appropriate method for addressing missing data that is either MAR or MCAR (Tabachnick \& 
Fidell, 2007). Multiple imputation is a method whereby missing values are imputed based on regression estimation procedures coupled with the addition of random error. After estimates have been generated for all of the missing values, a randomly drawn error value is then added to the regression-estimated imputed value, helping to account for uncertainty. In the present study, the following 23variables were used in the imputation model to estimate missing values: YLS/CMI 2.0 criminal need sub-score, YLS/CMI 2.0 total strength score, YLS/CMI 2.0 family strength score, YLS/CMI 2.0 education strength score, YLS/CMI 2.0 peer strength score, YLS/CMI 2.0 substance abuse strength score, YLS/CMI 2.0 leisure strength score, YLS/CMI 2.0 personality strength score, YLS/CMI 2.0 attitude strength score, YLS/CMI 2.0 criminal history risk score, YASI criminal need score, YASI overall protective score, YASI static protective score, YASI dynamic protective score, YASI family dynamic protective sub-score, YASI education dynamic protective sub-score, YASI social dynamic protective, YASI aggression dynamic protective subscore, YASI attitudes static protective sub-score, YASI attitudes dynamic protective sub-score, YASI skills dynamic protective sub-score, YASI employment static protective sub-score, and YASI employment dynamic protective sub-score. The aforementioned variables were included in order to get the most accurate estimates of missing data possible and were all variables that were likely to be related in some way to the domains that had missing data.

In the current study, 10 iterations of imputed data were produced (IBM Reference Guide, 2011). Analyses are performed separately for each imputed data set and resultant parameter estimates and standard errors are then pooled across the 10 imputed data sets when possible (Ml cannot be used with all statistical procedures and in some cases will not produce pooled estimates) (IBM Reference Guide, 2011).

Assumptions. All assumption testing was done by gender. Each of the subdomain and total YLS/CMI 2.0 scores and YASI sub-domain and total scores was examined for the presence of 
univariate outliers. Outliers were examined by transforming each value into a standardized $\mathrm{z}$-score using the critical values of +-3.29 (Tabachnick \& Fidell, 2007). Standardized values that were either above or below 3.29 were considered univariate outliers. Examination of z-scores revealed 2 outliers on YLS/CMI tool; both outliers (1 male; 1 female) were strength variables and were simply the only cases on those particular variables that possessed a strength (i.e., coded as 1). Due to the dichotomous nature of the variables, the values were not changed. Any analyses involving these two variables were restricted to descriptive as opposed to inferential statistics. In addition, 26 outliers emerged for the YASI sub-domain and total scores (17 male outliers; 9 female outliers). The outliers were brought within +-3 standard deviations of the mean for each variable.

The extent of multicollinearity between the variables was assessed using the tolerance statistic. A tolerance value below .1 is problematic (Field, 2005). All of the YLS/CMI 2.0 and YASI variables under consideration produced acceptable tolerance values. Normality was assessed through a visual examination of histograms, the Kolmogorov-Smirnov and Shapiro-Wilk statistical tests, as well as through a statistical examination of skewness and kurtosis. Skewness and kurtosis values were considered acceptable if they were within +-3.29 after being divided by the standard error (Tabachnick \& Fidell, 2007). The YASI variables revealed some problems with skewness and kurtosis. Nine YASI variables and one YLS/CMI variable evidenced positive skew and two YASI variables showed negative skew. Most variable skewness was resolved with a simple square root transformation. However, one variable required a log transformation to normalize the distribution. Transformations were not effective for the dynamic protective aggression sub-score, the static protective attitudes sub-score, or the static risk employment sub-score. After running analyses pre and post transformation, results showed no differences, thus the transformations for all variables were abandoned for ease of interpretation. No normality violations were evident for the YLS/CMI 2.0 variables. 
Controlling for potential confounds-Offence history, age, and race. Before the primary analyses could be conducted, it was first necessary to examine the extent to which male and female offenders may have differed on two potential confounds: age and race. An independent samples t-test was conducted to determine if gender differences in age existed on the resultant sample of 61 males and 59 females. The difference in age between males $(M=17)$ and females $(M$ $=16.77$ ) was non-significant $t(120)=1.093, p=.276$. Thus, age was not a confounding variable and there was no need to incorporate additional design or statistical controls.

Race was the final variable to be examined for being a potential. Recall that the racial composition was as follows: $4 \%$ Aboriginal, $29.7 \%$ African American, $1.4 \%$ Asian, $46.1 \%$ Caucasian, 1.8\% East Indian, 0.9\% Hispanic, and 15.5\% Other. In order to simplify results and ease interpretation, race was collapsed into a dichotomous variable (Caucasian vs. non-Caucasian). A chi-square test revealed that there were significant differences in race between males $(49 \%$ Caucasian) and females $(69 \%$ Caucasian $), X^{2}(1, N=120)=4.27, p=.039$. Therefore, race was used as a covariate in the moderated regression analyses.

\section{Descriptive Statistics}

Overall YLS/CMI 2.0 risk/need ratings by gender. The YLS/CMI 2.0 generates total risk/need levels (low, moderate, high, and very high) based on the sum of all 42 items in the tool. Risk/need level cut-off ranges differ for gender and location at time of assessment. For example, for males and females in a custodial setting cut-off ranges are as follows: low (0-19), moderate (20-29), high (30-36), and very high (37-42). The cut-off ranges for community males are as follows: low (0-9), moderate (10-21), high (22-31), and very high (32-42). The cut-off ranges for community females are slightly different: low (0-8), moderate (9-19), high (20-28), and very high (29-42) (Hoge \& Andrews, 2011). 
Overall, results show a slightly higher risk level for males in both community and custodial settings, but the results were not significant. Results are separated into custodial and community categories as well as males and females due to different cut-off ranges. Approximately three quarters of the entire sample came from a custodial setting ( 45 males; 41 females). Out of the male custodial sample, $16.27 \%(14)$ of youth were categorized as low risk, $29.1 \%(25)$ were categorized as moderate risk and $6.98 \%(6)$ scored high on the YLS/CMI 2.0. Out of the custodial female sample, $12.8 \%(11)$ of youth were categorized as low risk on the YLS/CMI 2.0, 25.6\% (22) were categorized as moderate risk, and $9.3 \%(8)$ fell into the high risk category. An independent samples t-test showed that the cummulative risk level on the YLS/CMI 2.0 did not differ significantly for males and females in the custodial sample, $t(84)=-.727, p=.47$.

The community sample was comprised of 16 females and 13 males. Out of the female community sample $12.5 \%$ (2) was categorized as low risk on the YLS/CMI 2.0,68.8\% (11) was moderate risk, $12.5 \%(2)$ was high risk and $6.3 \%$ (1) was very high risk. Finally, $46.2 \%(6)$ of the male community sample were classified as low risk, $30.8 \%(4)$ were classified as moderate risk, and $23.1 \%$ (3) were classified as high risk on the YLS/CMI 2.0. An independent samples t-test demonstrated that males and females in the community sample also did not significantly differ by risk category, $t(27)=-.643), p=.525$.

As Table 4 illustrates, the pooled results (derived through multiple imputation ${ }^{2}$ ) for an independent samples t-test determined that males pooled $M=20.79$ (range 20.67-20.83), $S D$ range $=8.42$ to 8.48 ) scored similarly to females pooled $M=21.09$ (range $21.04-21.28), S D$ range $=6.38$ to 6.44 ) on the YLS/CMI 2.0 total risk/need score $t(116)=(-2.08), p=.835$. Similarly, a series of independent samples t-tests also revealed that there were no significant differences between the

\footnotetext{
${ }^{2}$ SPSS does not pool standard deviations, therefore ranges will be reported throughout
} 
males and females on any of the eight individual need domains. Effect sizes (found in Table 4) also indicate no effect for all of the domains with one exception. The Cohen's $d$ calculation for the family domain indicates a small effect (.264). A post-hoc power analysis using the Daniel Soper post-hoc power calculator (www.danielsoper.com) was also conducted. With the current sample of 61 males and 59 females, the power analysis for the family domain indicated only a $30 \%$ chance of detecting a significant effect, which is poor compared to the usual standard of .80 for good statistical power. 
Table 4

Youth Level of Risk and Case Management Index Risk/Need Domain Mean Scores and Corresponding Descriptives by Gender.

\begin{tabular}{|c|c|c|c|c|c|c|c|}
\hline \multicolumn{3}{|c|}{$\begin{array}{c}\text { Males } \\
(n=61)\end{array}$} & \multicolumn{3}{|c|}{$\begin{array}{l}\text { Females } \\
(n=59)\end{array}$} & \multirow[b]{2}{*}{$\begin{array}{l}\text { Post-hoc } \\
\text { Power }\end{array}$} & \multirow[b]{2}{*}{ Cohen's d } \\
\hline $\begin{array}{l}\text { YLS/CMI } \\
\text { Domain }\end{array}$ & Mean (SD) & Range & Mean (SD) & Range & $t$ & & \\
\hline Legal History & $2.37(1.81-1.84)$ & 0 to 5 & $2.00(1.59-1.61)$ & 0 to 5 & 1.178 & .210 & .214 \\
\hline Family & $2.81(1.71-1.75)$ & 0 to 6 & $3.24(1.42-1.50)$ & 0 to 6 & -1.415 & .294 & .264 \\
\hline $\begin{array}{l}\text { Employment } \\
\text { \& Education }\end{array}$ & $2.66(1.80-1.86)$ & 0 to 6 & $2.82(1.77-1.85)$ & 0 to 6 & -.457 & .067 & .086 \\
\hline Peers & $2.59(1.19-1.20)$ & 0 to 4 & $2.81(1.18-1.20)$ & 0 to 4 & -.967 & .164 & .183 \\
\hline $\begin{array}{l}\text { Substance } \\
\text { Abuse }\end{array}$ & $2.41(1.62-1.68)$ & 0 to 5 & $2.63(1.49-1.56)$ & 0 to 5 & -.746 & .113 & .136 \\
\hline Leisure & $1.72(.90-.91)$ & 0 to 3 & $1.74(.88-.89)$ & 0 to 3 & -.177 & .033 & .022 \\
\hline Personality & $3.96(1.81-1.84)$ & 0 to 7 & $4.14(1.50-1.54)$ & 1 to 7 & -.597 & .142 & .106 \\
\hline Attitudes & $1.99(1.26 .1 .28)$ & 0 to 4 & $1.89(1.34-1.36)$ & 0 to 5 & .422 & .060 & .076 \\
\hline $\begin{array}{l}\text { Total } \\
\text { Risk/Need }\end{array}$ & $20.79(8.42-8.48)$ & 2 to 33 & $21.09(6.38-6.44)$ & 5 to 36 & -.208 & .041 & .04 \\
\hline
\end{tabular}

Note. $S D=$ Standard deviation ; the means are pooled as per multiple imputation; standard deviations are smallest and largest of all 10 imputed results; post-hoc power = two-tailed observation 
YASI pre-screen risk/need ratings by gender. The YASI pre-screen assessment includes nine domains: legal history, family, school, community and peers, alcohol and drugs, mental health, aggression, attitudes, and skills. As previously mentioned, the YASI pre-screen is used for triage purposes and designates the official risk categorization on the YASI measure. Appendix B provides the detailed breakdown of cut-off ranges for males and females on the YASI pre-screen. Results of an independent samples t-test revealed that males pooled $M=55.78$ (range 55.2357.09), $S D$ range $=21.74-23.29$ did not score differently from females pooled $M=60.39$ (range= $57.96-62.52), S D$ range $=16.55-19.78$ on the overall YASI pre-screen risk $t(118)=-1.138, p=$ .257. Furthermore, the YASI pre-screen categorized the males as follows: $3.3 \%(n=2)$ as low risk, 18 to $19.7 \%(n=10-11)$ as moderate risk, and 77 to $80.3 \%(n=47-49)$ as high risk. Out of the female sample, $1.7-3.4 \%(n=1-2)$ were categorized as low risk, $6.8-13.6 \%(n=4-8)$ were categorized as moderate risk, and $84.7-91.5 \%(n=50-54)$ were categorized as high risk as designated by the YASI pre-screen.

YASI full-assessment risk/need ratings by gender. The YASI full assessment is comprised of 10 individual domains: legal history, family, school, community and peers, alcohol and drugs, mental health, aggression, attitudes, skills, and employment and free time. Males pooled $M=154.64$ (range 146.62 to 157.09 ), $S D$ range $=49.23-54.18$ and females pooled $M=$ 166.29 (range $163.53-167.90, S D$ range $=38.27-46.83$ did not differ significantly on the overall risk score on the YASI measure $t(118)=(-1.356), p=.175$. Using the bonferroni correction for multiple comparisons the only domain evidencing differences was the family static risk domain where females scored significantly higher than males (See Table 5). However, 2 domains (family dynamic risk and education dynamic risk evidenced a small effect size in favour of females, while the p-value was insignificant. Furthermore, the peer static risk domain evidenced a small effect 
size in favour of males. The post-hoc power analyses suggest that the inconsistency between the p-values and effect sizes for these two domains is due to a small sample size. The YASI also provides cut-off values for each sub-domain to categorize youth into zero, low, moderate, or high risk or protection (See Appendix B). Figures 1 through 29 in Appendix B show the distributions of none, low, moderate, and high risk or protection for the current sample on each YASI domain. 
Table 5

Youth Assessment and Screening Inventory Risk/Need Domain Mean Scores and Corresponding Descriptives by Gender.

\begin{tabular}{|c|c|c|c|c|c|c|c|}
\hline & Males & & Female & & & & \\
\hline YASI Domain & Mean $(\mathrm{SD})$ & Range & Mean (SD) & Range & $t$ & Cohen's d & Post-hoc power \\
\hline \multicolumn{8}{|l|}{ Legal History } \\
\hline Static Risk & $20.07(11.03-11.60)$ & $0-41$ & $20.07(10-11.20)$ & $4-45$ & .003 & .00 & .025 \\
\hline $\begin{array}{l}\text { Dynamic } \\
\text { Risk }_{\mathrm{a}}\end{array}$ & -- & -- & - & -- & -- & -- & -- \\
\hline \multicolumn{8}{|l|}{ Family } \\
\hline Static Risk & $3.53(3.07-3.35)$ & $0-11$ & $5.93(5.74-6.15)$ & $0-13$ & $3.77^{-}$ & .48 & .74 \\
\hline $\begin{array}{l}\text { Dynamic } \\
\text { Risk }\end{array}$ & $16.73(7.0-8.75)$ & $0-36$ & $20.78(8.26-9.03)$ & $3-38$ & $2.561 * *$ & .45 & .68 \\
\hline \multicolumn{8}{|l|}{ Education } \\
\hline Static Risk & $2.39(2.28-2.47)$ & $0-5$ & $2.05(1.98-2.40)$ & $0-5$ & 1.345 & .14 & .12 \\
\hline $\begin{array}{l}\text { Dynamic } \\
\text { Risk }\end{array}$ & $8.012(7.01-7.30)$ & $0-26$ & $10.34(5.78-6.20)$ & $0-25$ & $-1.867^{*}$ & .34 & .45 \\
\hline \multicolumn{8}{|l|}{ Peer Relations } \\
\hline Static Risk & $5.72(3.68-3.94)$ & $0-11$ & $4.71(2.83-2.94)$ & $0-11$ & 1.556 & .29 & .35 \\
\hline $\begin{array}{l}\text { Dynamic } \\
\text { Risk }\end{array}$ & $17.12(6.8-7.14)$ & $0-28$ & $15.79(6.25-6.53)$ & $0-27$ & 1.087 & .19 & .18 \\
\hline
\end{tabular}

Note. $S D=$ standard deviation; presented means are pooled; standard deviations are smallest and largest of all 10 imputed results; post-hoc power $=$ two-tailed observation; ${ }^{* * *} \mathrm{p}<.001,{ }^{* *} \mathrm{p}<.01,{ }^{*} \mathrm{p}<.05$; a Values absent because domain does not exist. 
Table 5 Continued

\begin{tabular}{|c|c|c|c|c|c|c|c|c|}
\hline & Males & & Fen & nales & & & & \\
\hline YASI Domain & Mean $\quad(S D)$ & Range & Mean (SD) & & Range & $t$ & Cohen's d & Post-hoc power \\
\hline \multicolumn{9}{|l|}{ Substance Abuse } \\
\hline Static Risk & $3.73(3.68-3.8)$ & $0-7$ & $3.96(2.43-2.59)$ & & $0-7$ & -.591 & .09 & .07 \\
\hline $\begin{array}{l}\text { Dynamic } \\
\text { Risk }\end{array}$ & $11.12(6.34-6.43)$ & $0-20$ & $11.79(5.6-5.78)$ & & $0-20$ & -.719 & .12 & .09 \\
\hline \multicolumn{9}{|l|}{ Mental Health } \\
\hline Static Risk & $3.41(3.16-3.54)$ & $0-11$ & $4.97(3.57-4.13)$ & & $0-13$ & -2.211 & .04 & .04 \\
\hline $\begin{array}{l}\text { Dynamic } \\
\text { Risk }_{\mathrm{a}}\end{array}$ & -- & -- & -- & -- & $\begin{array}{l}- \\
-\end{array}$ & -- & & -- \\
\hline \multicolumn{9}{|l|}{ Aggression } \\
\hline Static Risk & $4.24(2.29-2.43)$ & $0-6$ & $4.29(2.32-2.44)$ & $0-6$ & -.110 & & .02 & .03 \\
\hline $\begin{array}{l}\text { Dynamic } \\
\text { Risk }\end{array}$ & $11.56(4.52-4.75)$ & $0-17$ & $11.87(3.87-4)$ & $0-17$ & -.394 & & .07 & .06 \\
\hline
\end{tabular}


Table 5 Continued

\begin{tabular}{|c|c|c|c|c|c|c|c|}
\hline & \multicolumn{2}{|l|}{ Males } & \multicolumn{3}{|c|}{ Females } & \multirow[b]{2}{*}{ Cohen's d } & \multirow[b]{2}{*}{ Post-hoc power } \\
\hline YASI Domain & Mean $(\mathrm{SD})$ & Range & $\begin{array}{l}\text { Mean } \\
\text { (SD) }\end{array}$ & Range & $t$ & & \\
\hline \multicolumn{8}{|l|}{ Attitudes } \\
\hline Static Risk & $.651(.617-.635)$ & $0-2$ & $.527(.520-.550)$ & $0-2$ & 1.098 & .21 & .21 \\
\hline $\begin{array}{l}\text { Dynamic } \\
\text { Risk }\end{array}$ & $20.88(9.07-9.83)$ & $3-35$ & $21.33(9.29-9.60)$ & $1-35$ & -.258 & .04 & .04 \\
\hline \multicolumn{8}{|l|}{ Skills } \\
\hline Static Risk & -- & -- & -- & -- & -- & - & -- \\
\hline $\begin{array}{l}\text { Dynamic } \\
\text { Risk }\end{array}$ & $18.81(9.34-10.46)$ & $0-34$ & $19.14(8.81-9.29)$ & $0-33$ & -.188 & .03 & .04 \\
\hline \multicolumn{8}{|l|}{$\begin{array}{l}\text { Employment \& } \\
\text { Free Time }\end{array}$} \\
\hline Static Risk & $.364(.650-.666)$ & $0-2$ & $.232(.206-.246)$ & $0-2$ & 1.202 & .26 & .29 \\
\hline $\begin{array}{l}\text { Dynamic } \\
\text { Risk }\end{array}$ & $3.62(2.30-2.36)$ & $0-7$ & $3.06(2.1-2.3)$ & $0-7$ & 1.345 & .24 & .26 \\
\hline
\end{tabular}




\section{Reliability Results}

Reliability was assessed in two ways. First, inter-rater agreement was assessed vis-à-vis the intra-class correlation coefficient (ICC). ICC's vary between -1 and 1 , with 0 indicating chance agreement and higher (positive) values indicating better agreement. According to the standards used by Parkerson, Broadhead, and Tse (1993), an ICC of less the .40 is "poor," between .40 and .75 is "adequate," and above .75 is "good." In particular, consistency ICC was

used, where results are based on whether both raters scored in the same direction-both raters did not have to provide identical ratings, but merely where one rater scored an item higher, the other rater did as well. Finally, the "Two-Way Mixed Model" for ICC's was used, making variability among the raters a secondary source of systematic variability.

Second, Chronbach's alpha was used to assess internal consistency. Cronbach's alpha usually ranges between 0 and 1 -the closer the Cronbach's alpha coefficient is to 1 , the greater the internal consistency of the measure. While there has been some debate over what an acceptable Cronbach's alpha level is, generally the following rules apply: <.5-unacceptable, $>$. 5poor, >.6-questionnable, > .7-acceptable, > .8-good, .9-excellent (George \& Mallery, 2003).

YASI. The YASI demonstrated good inter-rater reliability with ICC values for subdomains falling between .70 (substance abuse dynamic risk) and .95 (substance abuse static risk) (See Table 7) and between .74 (dynamic risk total) and .90 (static risk total) for Overall scores (See Table 6). However there were some domains that demonstrated lower inter-rater reliability (i.e. ICC values below .6) (See the social static risk domain, the skills dynamic risk domain, and the attitudes static protective domain in Table 6) ICC values on the low end ranged from -.035 to .555 . 
Table 6

Reliability Results: Intra-class Correlation Coefficients For Youth Assessment and Screening Inventory Total Domains

\begin{tabular}{ccc}
\hline & \multicolumn{2}{c}{ Intraclass Correlation Coefficients (ICCs) } \\
\hline YASI Domain & Single ICCs & Average ICCs \\
\hline Overall Protective & .787 & .881 \\
Dynamic Protective Total & .809 & .895 \\
Static Protective & .817 & .900 \\
Overall Risk & .871 & .931 \\
Static Risk Total & .909 & .952 \\
Dynamic Risk Total & .738 & .849 \\
\hline
\end{tabular}

Note. a "Average Measure Intraclass Correlation" is not relevant to this particular problem. It represents the estimate of the reliability if we averaged the scores of the two raters, and used that as a variable

Table 7

Reliability Results: Intra-class Correlation Coefficients For Youth Assessment and Screening Inventory Sub-domains

Intraclass Correlation Coefficients (ICCs)

\begin{tabular}{ccc}
\hline \multicolumn{1}{c}{ YASI Domain } & Single ICCs & Average ICCs $_{\mathbf{a}}$ \\
\hline Legal History & .704 & .826 \\
Static Risk & -- & -- \\
Dynamic Risk & & \\
Family & .814 & .897 \\
Static Risk & .501 & .668 \\
Dynamic Risk & .756 & .863 \\
Dynamic Protective & -- & - \\
Static Protective &
\end{tabular}

Note. a "Average Measure Intraclass Correlation" is not relevant to this particular problem. It represents the estimate of the reliability if we averaged the scores of the two raters, and used that as a variable 
Table 7 Continued

\begin{tabular}{|c|c|c|}
\hline \multicolumn{3}{|c|}{ Intraclass Correlation Coefficients (ICCs) } \\
\hline YASI Domain & Single ICCs & Average $\mathrm{ICCs} s_{\mathrm{a}}$ \\
\hline \multicolumn{3}{|l|}{$\overline{\text { Education }}$} \\
\hline Static Risk & .775 & .873 \\
\hline Dynamic Risk & .777 & .875 \\
\hline Dynamic Protective & .894 & .944 \\
\hline Static Protective & - & -- \\
\hline Static Protective & -- & - \\
\hline \multicolumn{3}{|l|}{ Substance Abuse } \\
\hline Static Risk & .949 & .974 \\
\hline Dynamic Risk & .702 & .825 \\
\hline Dynamic Protective & -- & -- \\
\hline Static Protective & -- & -- \\
\hline \multicolumn{3}{|l|}{ Mental Health } \\
\hline Static Risk & .783 & .878 \\
\hline Dynamic Risk & -- & -- \\
\hline Dynamic Protective & -- & - \\
\hline Static Protective & -- & \\
\hline Aggression & -- & - \\
\hline Static Risk & .463 & .633 \\
\hline Dynamic Risk & .720 & .837 \\
\hline Dynamic Protective & .691 & .818 \\
\hline Static Protective & -- & -- \\
\hline \multicolumn{3}{|l|}{ Attitudes } \\
\hline Static Risk & .723 & .839 \\
\hline Dynamic Risk & .775 & .873 \\
\hline Dynamic Protective & .752 & .859 \\
\hline Static Protective & $(-.035)$ & $(-.072)$ \\
\hline
\end{tabular}

a "Average Measure Intraclass Correlation" is not relevant to this particular problem. It represents the estimate of the reliability if we averaged the scores of the two raters, and used that as a variable 
Table 7 Continued

\begin{tabular}{lcc}
\hline \multicolumn{3}{c}{ Intraclass Correlation Coefficients (ICCs) } \\
\hline YASI Domain & Single ICCs & Average ICCs \\
\hline Skills & & \\
Static Risk & -- & - \\
Dynamic Risk & .555 & .714 \\
Dynamic Protective & .674 & .805 \\
Static Protective & -- & -- \\
Employment \& Free Time & & \\
Static Risk & .866 & .928 \\
Dynamic Risk & .453 & .623 \\
Static Protective & .923 & .960 \\
Dynamic Protective & .585 & .783 \\
\hline
\end{tabular}

a "Average Measure Intraclass Correlation" is not relevant to this particular problem. It represents the estimate of the reliability if we averaged the scores of the two raters, and used that as a variable

YLS/CMI 2.0. With one exception, the majority of YLS/CMI 2.0 risk/need subdomains demonstrated adequate or good inter-rater reliability (i.e., all $>.50$ ) which is consistent with past studies assessing the reliability of the YLS/CMI 2.0 (See Table 8). The employment and education domain demonstrated poor inter-rater reliability with an ICC of only.264. In addition, none of the YLS/CMI 2.0 strength domains had acceptable ICCs (i.e., all <.326) with the exception of the Family Strength variable indicating an adequate ICC of .579 and the total strength score with an adequate ICC of .577 .

Table 8

Summary of Intra-class Correlations and Alphas for Scores on YLS/CMI Subdomains

Intraclass Correlation Coefficients (ICCs)

\begin{tabular}{|c|c|c|}
\hline & Single ICCs & Average $\mathrm{ICCs}_{\mathrm{a}}$ \\
\hline YLS/CMI Risk Sub-domains & & \\
\hline Criminal History & .846 & .917 \\
\hline Family Need & .645 & .784 \\
\hline
\end{tabular}


Table 8 Continued

\begin{tabular}{|c|c|c|}
\hline & \multicolumn{2}{|c|}{ Intraclass Correlation Coefficients (ICCs } \\
\hline & Single ICCs & Average $\mathrm{ICCs}_{\mathbf{a}}$ \\
\hline \multicolumn{3}{|l|}{ YLS/CMI Risk Sub-domains } \\
\hline $\begin{array}{l}\text { Employment \& } \\
\text { Education Need }\end{array}$ & .264 & .417 \\
\hline Peers Need & .557 & .715 \\
\hline $\begin{array}{l}\text { Substance Abuse } \\
\text { Need }\end{array}$ & .777 & .875 \\
\hline Leisure & .815 & .898 \\
\hline Personality & .664 & .798 \\
\hline Attitudes & .513 & .678 \\
\hline \multicolumn{3}{|l|}{ Strength Sub-domains } \\
\hline Family Strength & .579 & .733 \\
\hline Education Strength & .141 & .247 \\
\hline Peer Strength & .186 & .314 \\
\hline $\begin{array}{l}\text { Substance Abuse } \\
\text { Strength }\end{array}$ & -.050 & -.105 \\
\hline Leisure Strength & .250 & .400 \\
\hline Personality Strength & -.069 & -.148 \\
\hline Attitude Strength & .326 & .492 \\
\hline Total Risk/Need & .776 & .874 \\
\hline Strength Total & .577 & .732 \\
\hline
\end{tabular}

a "Average Measure Intraclass Correlation" is not relevant to this particular problem. It represents the estimate of the reliability if we averaged the scores of the two raters, and used that as a variable

Research Question 1: Do the number and types of strengths as measured on the YLS/CMI

\section{0 and YASI respectively vary as a function of gender?}

YLS/CMI 2.0 strengths by gender. The strength component of the YLS/CMI 2.0, in contrast to the risk component, is comprised of seven dichotomously scored (yes/no) domains: family, education, peers, substance abuse, leisure, personality, and attitudes. The relationship between gender and strengths was analyzed in one of two ways. First, to examine whether the types of strengths on the YLS/CMI 2.0 vary by gender, each of the seven dichotomously scored YLS/CMI 2.0 strengths was cross-tabulated with gender. Eight chi-square analyses revealed no 
significant findings (all $p$ 's $>.05)$ (See Table 9)

Table 9.

Nature of Youth Level of Service Strengths by Gender.

\begin{tabular}{lccc}
\hline $\begin{array}{l}\text { YLS/CMI Strength } \\
\text { Domain }\end{array}$ & $\begin{array}{c}\text { Strength Present } \\
\text { Males }(\mathrm{n}=61) \\
\%(\mathrm{n})\end{array}$ & $\begin{array}{c}\text { Strength Present } \\
\text { Females }(\mathrm{n}=59) \\
\%(\mathrm{n})\end{array}$ & $X^{2}$ \\
\hline Family & $36(22)$ & $23.7(14)$ & 4.185 \\
Education & $31.1(19)$ & $33.8(20)$ & 2.107 \\
Peers & $16.4(10)$ & $8.5(5)$ & 3.722 \\
Substance Abuse & $13.1(8)$ & $8.5(5)$ & 3.698 \\
Leisure & $31.1(19)$ & $20.3(12)$ & 3.836 \\
Personality & $6.6(4)$ & $6.8(4)$ & 2.004 \\
Attitude & $13.1(8)$ & $10.2(6)$ & 2.253 \\
\hline
\end{tabular}

Second, to address the question as to whether one gender possesses more cumulative strengths than the other, an independent samples $t$-test was used to compare males and females on the YLS/CMI total strength score. The independent samples $t$-test revealed that there were also no significant findings when comparing the male cumulative strength score (multiple imputed pooled $M=1.467$, range $M=1.446-1.493, S D=1.43-1.45)$ to the female cumulative strength score (pooled $M=1.143$, range $M=1.128-1.162, S D=1.25-1.27$ ) on YLS/CMI $2.0, t(118)=(1.31), p$ $=.191$.

YASI "protective factors" by gender. The protective component of the YASI is designed similarly to the risk component, where protection is assessed on each of the 10 individual domains, as well as at the total level. In contrast, the YASI pre-screen does not take into account protective factors. Each protective domain is accompanied by predefined cut-off 
ranges that differ by gender (please refer to Appendix B for specific cut-off ranges). Protective scores are meant to be used for case management and do not impact risk scores in practice. As opposed to the YLS/CMI 2.0, there were 3 unique "protective" domains on the YASI where males and females showed significant differences. A series of independent samples $t$ - tests $^{3}$ revealed that males and females did not differ significantly on any of the protective domains of the YASI after applying the bonferroni correction for multiple comparisons (See Table 10). However 3 domains evidenced small effects in terms of Cohen's $d$ in favour of males (family dynamic protective, education dynamic protective, and aggression dynamic protective). In addition, there were no differences between males pooled $M=31.57$ (range 31.09-32.55), $S D$ range $=18.87-19.18$ and females pooled $M=26.99$ (range $26.00-27.37$ ), $S D$ range $=14.87-15.21$ on the YASI total protective score, $t(118)=(1.98), p=.15($ See Table 10). However, the YASI total protective score also showed a small effect in favour of males.

\section{Research Question 2: How well do the YLS/CMI 2.0 and YASI strengths-both at the composite and domain levels - correlate with each other (where comparable) for males and \\ females separately?}

The YASI and YLS/CMI 2.0 measures do not consist of parallel domains in all cases; therefore, comparisons using Pearson's correlation coefficient were made where it was logical to do so. Specifically, we wanted to know the following: if the YASI dynamic protective family domain correlated with the YLS/CMI 2.0 family strength domain; if the YASI dynamic protective education domain correlated with the YLS/CMI 2.0 education strength domain; if the YASI dynamic protective peer relations domain correlated with the YLS/CMI 2.0 peer strength domain; if the YASI static and dynamic protective attitudes domains correlated with the YLS/CMI 2.0

\footnotetext{
${ }^{3}$ t-tests were used as opposed to a MANOVA (the ideal analysis) as SPSS version 19 does not allow a MANOVA analysis on imputed data.
} 
Table 10

Nature of Youth Assessment Screening Inventory Strengths by Gender.

\begin{tabular}{|c|c|c|c|c|c|c|c|}
\hline & \multicolumn{2}{|l|}{ Males } & \multicolumn{2}{|c|}{ Females } & \multirow[b]{2}{*}{$t$} & \multirow[b]{2}{*}{$\begin{array}{c}\text { Post-hoc } \\
\text { power }\end{array}$} & \multirow[b]{2}{*}{ Cohen's $d$} \\
\hline YASI Domain & Mean (SD) & Range & Mean (SD) & Range & & & \\
\hline Legal History & -- & $\cdots$ & - & - & - & - & -- \\
\hline \multicolumn{8}{|l|}{ Family } \\
\hline Static Protective & $\cdots$ & -- & -- & -- & - & -- & -- \\
\hline Dynamic Protective & $5.72(4.06-4.34)$ & $1-16.5$ & $4.09(2.95-3.30)$ & $1-16$ & 2.248 & .63 & .42 \\
\hline \multicolumn{8}{|l|}{ Education } \\
\hline Static Protective & - & -- & -- & -- & - & -- & - \\
\hline Dynamic Protective & $8.33(5.02-5.16)$ & $0-16$ & $6.81(4.49-4.71)$ & $0-16$ & 1.69 & .39 & .31 \\
\hline \multicolumn{8}{|l|}{ Peer Relations } \\
\hline Static Protective & -- & - & -- & -- & -- & -- & -- \\
\hline Dynamic Protective & $3.63(3.64-3.73)$ & 0.13 .5 & $3.10(3.08-3.26)$ & $0-13$ & .828 & .13 & .15 \\
\hline Substance Abuse & - & - & -- & -- & - & $\cdots$ & - \\
\hline Mental Health & -- & -- & - & -- & -- & - & -- \\
\hline \multicolumn{8}{|l|}{ Aggression } \\
\hline Static Protective & -- & & - & & - & $-\cdot$ & -- \\
\hline Dynamic Protective & $.84(1.62-1.68)$ & $0-5$ & $.34(.90-1.02)$ & $0-5$ & 1.95 & .50 & .36 \\
\hline \multicolumn{8}{|l|}{ Attitudes } \\
\hline Static Protective & $.33(.67-.71)$ & $0-2$ & $.46(.76-.81)$ & $0-2$ & -.913 & .15 & .17 \\
\hline Dynamic Protective & $3.66(4.06-4.22)$ & $0-16$ & $3.50(3.93-4.07)$ & $0-16$ & -.204 & .04 & .04 \\
\hline
\end{tabular}

Note. $S D=$ standard deviation; presented means are pooled; standard deviations are smallest and largest of all 10 imputed results; post-hoc power $=$ two-tailed observation. 
Table 10 Continued

\begin{tabular}{|c|c|c|c|c|c|c|c|}
\hline & Males & & Femal & & & & \\
\hline YASI Domain & Mean (SD) & Range & Mean (SD) & Range & $t$ & $\begin{array}{c}\text { Post-hoc } \\
\text { power }\end{array}$ & Cohen's d \\
\hline \multicolumn{8}{|l|}{ Skills } \\
\hline Static Protective & -- & -- & -- & - & - & -- & -- \\
\hline Dynamic Protective & $4.68(5.68-5.89)$ & $0-22$ & $4.20(5.23-5.45)$ & $0-22$ & .447 & .06 & .08 \\
\hline \multicolumn{8}{|l|}{ Employment \& Free Time } \\
\hline Static Protective & $2.26(1.92-2.06)$ & $0-5$ & $2.17(1.91-1.97)$ & $0-5$ & .234 & .04 & .04 \\
\hline Dynamic Protective & $2.57(2.09-2.16)$ & $0-8$ & $2.41(1.67-1.73)$ & $0-6$ & .452 & .06 & .08 \\
\hline Overall Protective & $30.57(18.87-19.18)$ & $7-87$ & $26.99(14.87-15.21)$ & $1-63$ & 1.98 & .29 & .26 \\
\hline
\end{tabular}

Note. $S D=$ standard deviation; presented means are pooled; standard deviations are smallest and largest of all 10 imputed results; post-hoc power $=$ two-tailed observation. 
attitudes domain; if the YASI static protective and dynamic protective employment and free time domains correlated with the YLS/CMI 2.0 leisure domain; and finally, if the YASI overall protective score domain correlated with the YLS/CMI 2.0 overall strength domain.

To determine whether the magnitude of the correlations between the strength domains on the YASI and YLS/CMI 2.0 were significantly different between males and females, two approaches were taken. The first approach involved the inspection of confidence intervals ${ }^{4}$, where overlapping intervals for males and females would indicate that any observed gender differences were non-significant (Lane, 2007). However, in order to capture differences across gender that may have been disguised by confidence intervals, a second approach was taken which involved transforming Pearson's $r$ into Fisher's $Z$ (the $r$ to $z$ transformation) using an online calculator to calculate the differences between the $\mathrm{Z}$ values (www.quantpsy.org) (Cohen, 1992; Lowry, 2012). The $r$ to $z$ transformation allows an inspection of Fisher's $Z$ to determine whether the difference between the male and female correlation coefficients on each domain were significant (i.e., falling above or below the 1.96 threshold).

Overall, out of the eight hypothesized comparisons conducted, six showed evidence of congruence using Fisher's $Z$ values and all eight comparisons showed congruence using 95\% confidence intervals. The YASI and YLS/CMI 2.0 family strength domains were moderately positively correlated with each other for males $(r=.394, p=.004)$ and females $(r=.342, p=$ $.018)$. However, the overlapping confidence intervals as well as non-significant Fisher's $Z$ values indicate that the differences between males and females are not significant ${ }^{5}$. In addition, the YASI and YLS/CMI 2.0 education domains were weakly positively correlated for males $(r=$ $.107, p=.413)$, but moderately positively correlated for females $(r=.311, p=.019)$; differences

\footnotetext{
${ }^{4}$ The following equation was used in the calculation of confidence intervals: $[z+/-(1.96)(\mathrm{SE})]$

${ }^{5}$ See Table 11 for $95 \%$ confidence intervals and Fisher's $Z$ values
} 
between males and females in the comparison of the YASI and YLS/CMI 2.0 education domains were non-significant. Differences between males who displayed a moderate positive correlation $(r=.494, p<.001)$ and females, who displayed a weak positive correlation $(r=.083, p=.546)$ were evident in the comparison of the YASI and YLS/CMI 2.0 peer domains; although confidence intervals overlapped slightly, the Fisher's $Z$ value was significant (See Table 11). Similarly, differences between males $(r=.051, p=.814)$ (weak positive correlation) and females $(r=.505, p<.001)$ (moderate positive correlation) were significant in comparison of the YASI static protective attitudes domain (but not the dynamic protective attitudes domain) and the YLS/CMI 2.0 attitudes domain; confidence intervals overlapped slightly, but the Fisher's Z value indicated significance (See Table 11). No significant differences were evident across gender in the comparison of the YASI employment and free time static and dynamic protective domains to the YLS/CMI 2.0 leisure domain. Males showed a weak positive correlation between the YASI static protective employment and free time domain and the YLS/CMI 2.0 leisure domain $(r=$ $.096, p=.470$ ) and females showed a weak negative correlation between the same two domains $(r=-.115, p=.405)$

Similarly, differences were non-significant between males $(r=.340, p=.008)$ and females $(r=.083, p=.885)$ in the comparison of the YASI dynamic protective employment and free time domain and the YLS/CMI 2.0 leisure domain. Finally, differences were also nonsignificant between males $(r=.541, p=<.001)$ (moderate positive correlation) and females $(r=$ $.353, p=.007)$ (moderate positive correlation) in the comparison of the YASI overall protective score and the YLS/CMI 2.0 total strengths score. In addition to the a priori comparisons that seemed logical to make between the YASI and YLS/CMI 2.0 strength domains, there were two additional comparisons at the domain level where males and females differed significantly. In the comparison between the YASI dynamic protective peer domain and the YLS/CMI 2.0 education 
Table 11

Relationship Between Youth Level of Service and Youth Assessment Screening Inventory Strength Domains

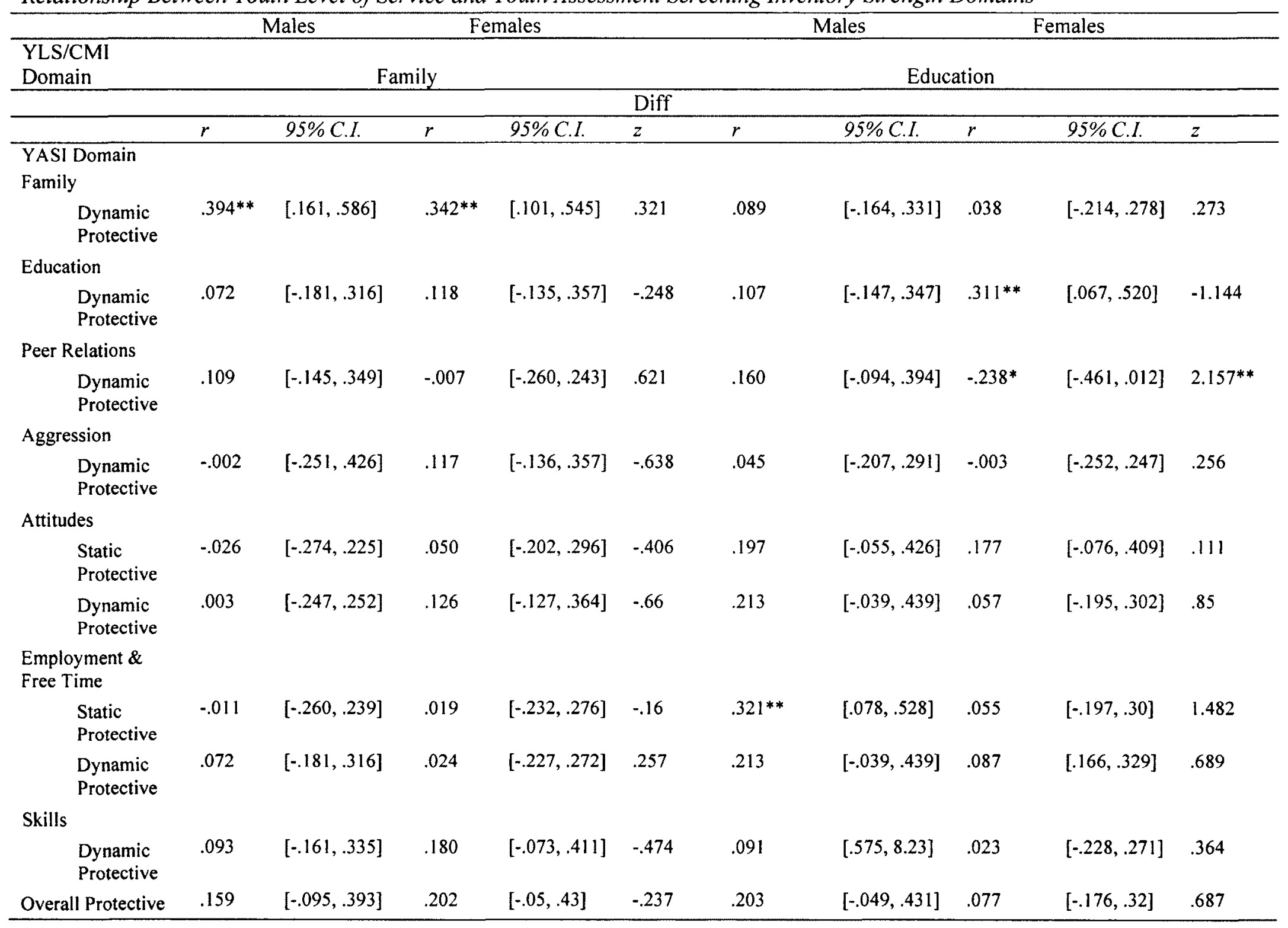


Table 11 Continued

\begin{tabular}{|c|c|c|c|c|c|c|c|c|c|c|}
\hline 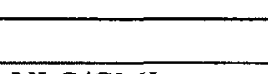 & \multicolumn{2}{|c|}{ Males } & \multicolumn{2}{|c|}{ Females } & \multicolumn{4}{|c|}{ Males } & \multicolumn{2}{|l|}{ Females } \\
\hline \multirow{3}{*}{ Domain } & \multicolumn{4}{|c|}{ Peers } & \multicolumn{6}{|c|}{ Leisure } \\
\hline & & & & & Diff & & & & & Dift \\
\hline & $r$ & $95 \%$ C.I. & $r$ & $95 \%$ C.I. & $z$ & $r$ & $95 \%$ C.I. & $r$ & $95 \%$ C.I. & $z$ \\
\hline \multicolumn{11}{|l|}{ YASI Domain } \\
\hline \multicolumn{11}{|l|}{ Family } \\
\hline $\begin{array}{l}\text { Dynamic } \\
\text { Protective }\end{array}$ & $.313^{* *}$ & {$[.069, .518]$} & -.148 & {$[-.383, .290]$} & $2.525 * *$ & $.229^{*}$ & {$[-.022, .453]$} & .091 & {$[-.163 .333]$} & .757 \\
\hline \multicolumn{11}{|l|}{ Education } \\
\hline $\begin{array}{l}\text { Dynamic } \\
\text { Protective }\end{array}$ & .210 & {$[-.042, .437]$} & .044 & {$[-.208, .290]$} & .903 & .155 & {$[-.099, .389]$} & -.025 & {$[-.273, .226]$} & .968 \\
\hline \multicolumn{11}{|l|}{ Peer Relations } \\
\hline $\begin{array}{l}\text { Dynamic } \\
\text { Protective }\end{array}$ & $.494 * * *$ & {$[.278, .662]$} & .083 & {$[-.170, .326]$} & $2.445 * *$ & $.401^{* * *}$ & {$[.168, .592]$} & -.404 & {$[-.593,-.171]$} & $4.554 * * *$ \\
\hline \multicolumn{11}{|l|}{ Aggression } \\
\hline $\begin{array}{l}\text { Dynamic } \\
\text { Protective }\end{array}$ & .155 & {$[-.099, .389]$} & -.132 & {$[-.370, .121]$} & 1.543 & .154 & {$[-.10, .388]$} & -.018 & {$[-.266, .233]$} & .925 \\
\hline \multicolumn{11}{|l|}{ Attitudes } \\
\hline $\begin{array}{l}\text { Static } \\
\text { Protective }\end{array}$ & -.021 & {$[-.269, .269]$} & -.110 & {$[-.350, .360]$} & .477 & -.024 & {$[-.272, .227]$} & -.025 & {$[-.273, .226]$} & .005 \\
\hline $\begin{array}{l}\text { Dynamic } \\
\text { Protective }\end{array}$ & $.224^{*}$ & {$[-.027, .449]$} & -.153 & {$[-.388, .388]$} & $2.039 * *$ & .149 & {$[-.105, .384]$} & .063 & {$[-.190,-.063]$} & .465 \\
\hline \multicolumn{11}{|l|}{$\begin{array}{l}\text { Employment \& } \\
\text { Free Time }\end{array}$} \\
\hline $\begin{array}{l}\text { Static } \\
\text { Protective }\end{array}$ & -.060 & {$[-.305, .193]$} & .167 & {$[-.086, .40]$} & -1.22 & .096 & {$[-.158, .337]$} & -.115 & {$[-.355, .138]$} & 1.131 \\
\hline $\begin{array}{l}\text { Dynamic } \\
\text { Protective }\end{array}$ & .045 & {$[-.207, .291]$} & .114 & {$[-.140, .353]$} & -.371 & $.340^{* *}$ & {$[.099, .543]$} & .083 & {$[-.170, .326]$} & 1.446 \\
\hline \multicolumn{11}{|l|}{ Skills } \\
\hline $\begin{array}{l}\text { Dynamic } \\
\text { Protective }\end{array}$ & .220 & {$[.031, .445]$} & -.153 & {$[-.388, .388$} & $2.017^{* *}$ & .191 & {$[-.059, .420]$} & .129 & {$[-.124, .367]$} & .34 \\
\hline Overall Protective & $.347^{* *}$ & {$[.107, .549]$} & -.063 & {$[-.308, .190]$} & $2.269^{* *}$ & $.298^{* *}$ & {$[.052, .509]$} & .034 & {$[-.217, .281]$} & 1.459 \\
\hline
\end{tabular}


Table 11 Continued

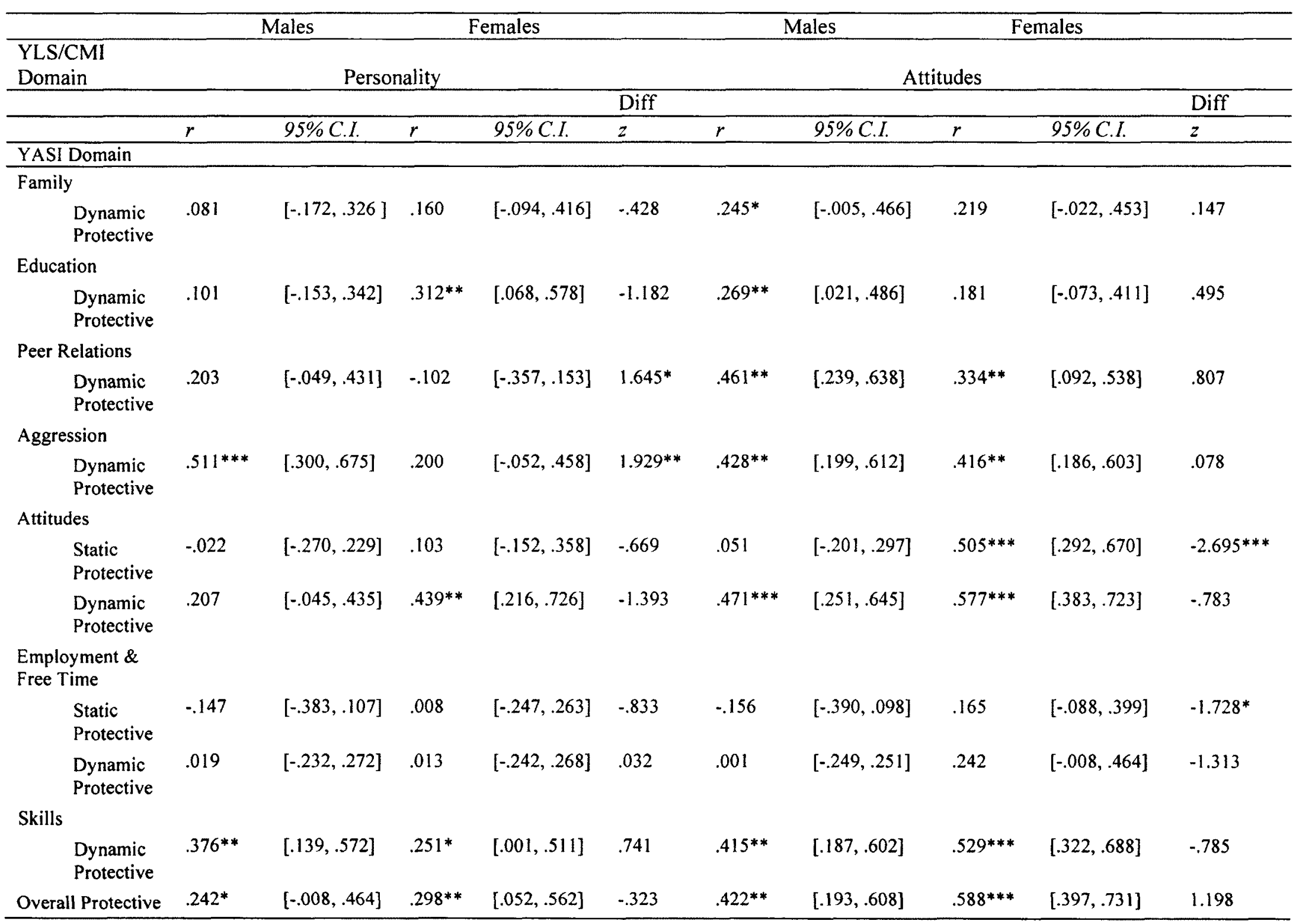


Table 11 Continued

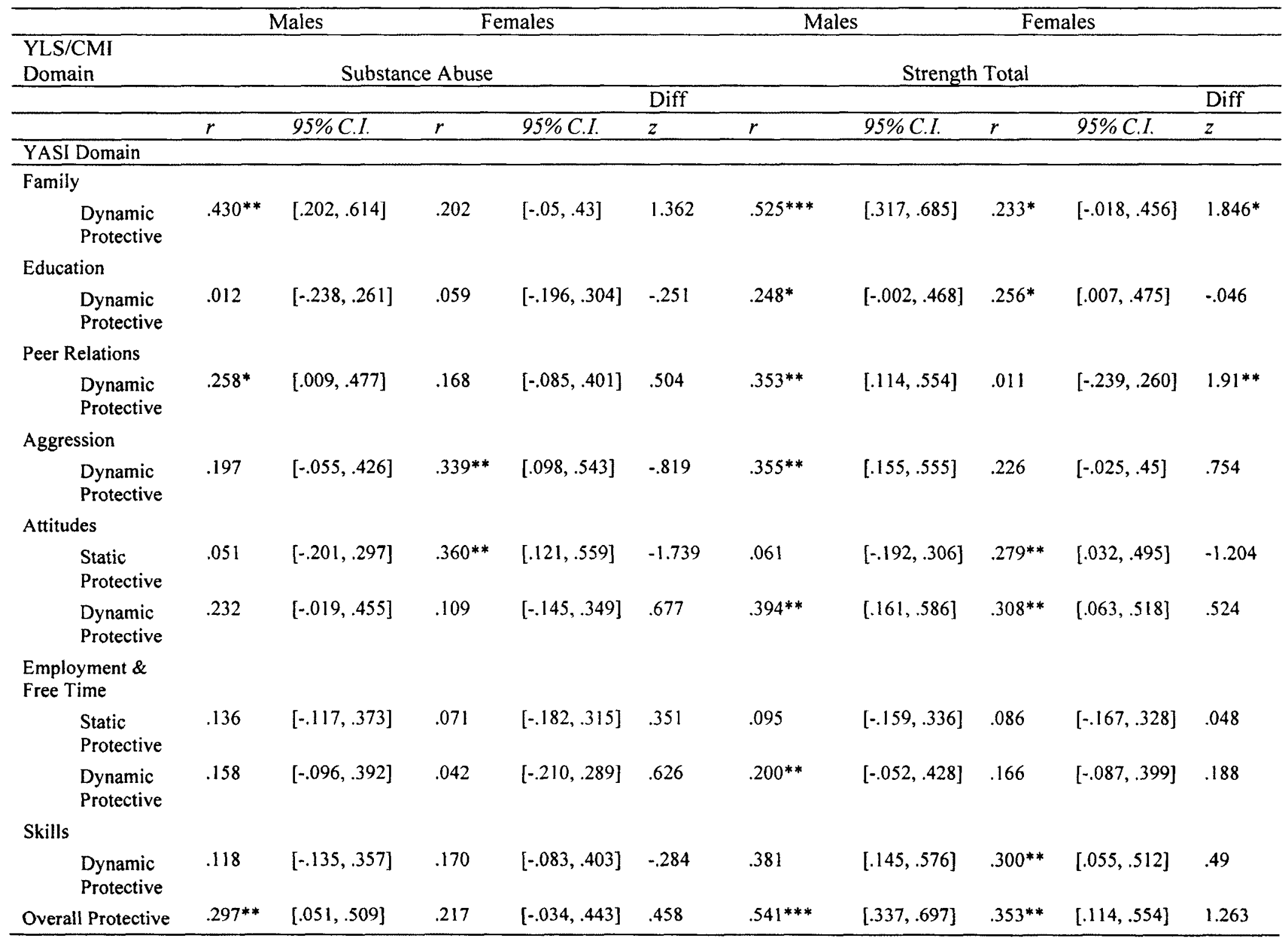


domain, males showed a weak positive correlation $(r=.160, p=.220)$ and females showed a moderate negative correlation $(r=-.238, p=.08)$; although confidence intervals overlapped, the Fisher's $Z$ value was significant (See Table 11). Furthermore, the correlations between the YASI dynamic protective peer domain and the YLS/CMI 2.0 leisure domain were also significantly different for males who demonstrated a moderate positive correlation $(r=.401, p=.001)$ and females who demonstrated a weak negative correlation $(r=-.040, p=.776)$ between the two domains; the difference is observed in both non-overlapping confidence intervals and a significant Fisher's Z value (See Table 11).

\section{Research Question 3: Do the YLS/CMI 2.0 strengths add incrementally to the post-diction} of criminal history above and beyond risk and is the relationship moderated by gender?

To determine if YLS/CMI strengths can post-dict YLS/CMI 2.0 criminal history and if strengths work differently in post-dicting criminal history for males and females, while taking into account the confounding variables of race and YLS/CMI 2.0 need, a hierarchical moderated regression model was used. In the regression model, the covariates (race and YLS/CMI 2.0 need) are entered in the first step. The second step of the regression model is where the predictors (sex and YLS/CMI 2.0 strengths) are entered. Finally, the interaction between sex and YLS/CMI 2.0 strengths is entered as the last step in the regression. The moderated hierarchical regression revealed that model 3 (the final step including the interaction term) was significant $\left(R^{2}=.344, F\right.$ $(2,114)=10.82, p<.001)$. However, the only variable accounting for significant variance in the model at every step of the regression was YLS/CMI 2.0 need (See Table 12). Overall, gender and YLS/CMI 2.0 strengths, along with the covariates of race and YLS/CMI 2.0 need accounted for $34.4 \%$ of the variance in YLS/CMI 2.0 criminal history. The interaction term was not significant. Additionally, the corresponding effect size, Cohen's $f^{2}$ was calculated using an online calculator (www.danielsoper.com) for determining the effect size of the difference between model 2 
(includes the covariates and 2 predictors) and model 3 (includes the interaction of the predictors). Notably, no interaction effect was present $\left(f^{2}=.003\right)$ between models 2 and 3 . Stated differently, the semi-partial correlation of $-.016\left(s r^{2}=.00026\right)$, representing the interaction between gender and YLS/CMI 2.0 strengths only accounts for $.026 \%$ unique variance in $\mathrm{R}^{2}$. Meanwhile, on its own, gender accounts for $1.9 \%$ of the unique variance in $\mathrm{R}^{2}\left(s r^{2}=.019\right)$ and strengths on their own accounted for $0.23 \%$ of unique variance in $\mathrm{R}^{2}\left(\mathrm{sr}^{2}=.002\right)$. Finally, YLS/CMI need accounts for nearly $20.0 \%$ of unique variance in $\mathrm{R}^{2}$ in the final hierarchical regression model $\left(s r^{2}=.198\right)$ and race accounted for $.036 \%\left(\mathrm{sr}^{2}=.00036\right)$. Although gender and YLS/CMI strengths accounted for some of the variance in YLS/CMI 2.0 criminal history, the variance was not significant over and above YLS/CMI 2.0 criminal need (See Table 12).

Research Question 4: Do the YASI strengths add incrementally to the post-diction of criminal history above and beyond risk and is the relationship moderated by gender?

To determine if YASI strengths can post-dict YLS/CMI 2.0 criminal history and if strengths work differently in post-dicting criminal history for males and females, while taking into account the confounding variables of race and YASI need, a hierarchical moderated regression model was used. In the regression model, the covariates (race and YASI need) are entered in the first step. The second step of the regression model is where the predictors (sex and YASI strengths) are entered. Finally, the interaction between sex and YASI strengths is entered as the last step in the regression. The moderated hierarchical regression revealed that the final model was significant $\left(R^{2}=.166, F(2,114)=2.86, p<.05\right)$. Collectively, gender and YASI strengths, along with the covariates of race and YASI need accounted for $16.6 \%$ of the variance in YLS/CMI 2.0 criminal history. Cohen's $f^{2}$ was calculated using an online calculator (www.danielsoper.com) for determining the effect size of the difference between Model 2 (includes the covariates and 2 predictors) and Model 3 (includes the interaction of the predictors). 
Table 12

Gender Moderated Regression Results for Youth Level of Service Strength Total Post-dicting Youth Level of Service Legal History Over and Above Criminal Need and Race

\begin{tabular}{|c|c|c|c|}
\hline & $\beta$ & $\begin{array}{c}B \\
\text { (Pooled) } \\
\end{array}$ & $\begin{array}{c}\text { SE (B) } \\
\text { (Pooled) }\end{array}$ \\
\hline \multicolumn{4}{|l|}{ Model 1} \\
\hline Race & $.037[-.015-.021]$ & $.044[-.052-.154]$ & $.284[.272-.286]$ \\
\hline YLS/CMI Need & $.570^{* * *}\left[.521^{* * *}-.541^{* * *}\right]$ & $.144[.141-.149]$ & $.021[.021-.022]$ \\
\hline \multicolumn{4}{|l|}{ Model 2} \\
\hline Race & $.011[-.044-.008]$ & $-.063[-.153-.029]$ & $.287[.277-.293]$ \\
\hline YLS/CMI Need & $\left..558^{* * *}[.49]^{* * *}-.524^{* * *}\right]$ & $.139[.135-.147]$ & $.024[.024-.025]$ \\
\hline YLS/CMI Strength Total & $-.037[-.081-(-.022)]$ & $-.072[-.105-(-.055)]$ & $.116[.112-.115]$ \\
\hline Sex & $-.147[-.160-(-.131)]$ & $-.509[-.549-(-.451)]$ & $.281[.273-.286]$ \\
\hline \multicolumn{4}{|l|}{ Model 3} \\
\hline Race & $.014[-.042-.008]$ & $-.060[-.146-.029]$ & $.288[.278-.293]$ \\
\hline YLS/CMI Need & $.572^{* * *}\left[-.492^{* * *}-.557 * * *\right]$ & $.140[.136-.150]$ & $.025[.024-.025]$ \\
\hline YLS/CMI Strength Total & $.012[-.075-.022]$ & $-.052[-.097-(-.014)]$ & $.158[.150-.157]$ \\
\hline Sex & $-.097[-.154-(-.092)]$ & $-.455[-.546-(-.318)]$ & $.397[.384-.403]$ \\
\hline Strength Total * Sex & $-.079[-.068-(-.001)]$ & $-.042[-.081-(-.002)]$ & $.213[.208-.217]$ \\
\hline
\end{tabular}

Note. Outcome variable is YLS/CMI 2.0 criminal history. $\beta$ is a standardized coefficient and $B$ is an unstandardized coefficient. SE $=$ standard error. For cases in which the estimates produced by the imputed datasets did not differ, the number is presented in place of the range. Model $1 R^{2}=.321$ (range of $.270-.305$ for multiple imputations). Model $1 F=25.06, p<.001$ (range of $21.67-25.42$ for multiple imputations). Model $2 R^{2}=.342$ (range of $.297-.322$ for multiple imputations). Model $2 F=12.74, p<.001$ (range of 12.03 13.54 for multiple imputations). Model $3 R^{2}=.344$ (range of $.297-.324$ for multiple imputations). Model $2 F=10.82, p<.001$ (range of $9.54-10.42$ for multiple imputations). ${ }^{*} p<.05{ }^{* *} p<.01^{* * *} p<.001$. 
No effect was present $\left(f^{2}=.014\right)$ between models 2 and 3 , meaning that there was no gender by strength interaction effect.

In the first step of the hierarchical regression, YASI need accounts for a significant amount of variance in YLS/CMI 2.0 criminal history, while race did not account for a significant portion of the variance in the dependent variable (See Table 13). However, in the second step of the regression model, where YASI strengths are added, YASI need no longer accounts for a significant proportion of variance in YLS/CMI 2.0 criminal history, while YASI strengths (multiply imputed ranges) are accounting for a significant proportion of the variance in criminal history (See Table 13). In the third step, with a semi-partial correlation of $.105\left(s r^{2}=.011\right)$, the interaction between gender and YASI total strengths only accounts for $1.1 \%$ unique variance in $\mathrm{R}^{2}$. Meanwhile, on its own, gender accounts for $2.66 \%$ of the unique variance in $\mathrm{R}^{2}\left(s r^{2}=.027\right)$ and strengths account for $5.15 \%$ of unique variance in $\mathrm{R}^{2}\left(\mathrm{sr}^{2}=.052\right)$. Finally, YASI need accounts for almost no unique variance in $\mathrm{R}^{2}$ in the final hierarchical regression model $\left(s r^{2}=\right.$ $.000004)$ while race accounts for $.52 \%$ of unique variance $\left(\mathrm{sr}^{2}=.0052\right)$.

\section{Discussion}

This study empirically investigated a collection of otherwise neglected facets about youth in conflict with the law. While countless studies seek to examine the way current assessment tools quantify criminal risk, this thesis is the first to addresses the way a "gold-standard" risk assessment tool (the YLS/CMI2.0) quantifies strengths and is among the first to investigate a new assessment tool (the YASI) that claims to provide a more comprehensive assessment of risk and protective factors for the young offender population. The investigation also highlights gender differences, or similarities in every research question it sought to answer. Using a gender informed approach, four research objectives were investigated using a sample of justice involved youth $(N=120 ;$ Males $=61$, Females $=59)$ in the Greater Toronto and Ottawa areas. 
Table 13

Gender Moderated Regression Results for Youth Assessment and Screening Inventory Protective Total Post-dicting Youth Level of Service Legal History Over and Above Criminal Need and Race

\begin{tabular}{|c|c|c|c|}
\hline & $\beta$ & $\begin{array}{c}B \\
\text { (Pooled) } \\
\end{array}$ & $\begin{array}{c}\text { SE (B) } \\
\text { (Pooled) }\end{array}$ \\
\hline \multicolumn{4}{|l|}{ Model 1} \\
\hline Race & $.035[-.027-.021]$ & $.012[-.095-.125]$ & $.335[.322-.384]$ \\
\hline YASI Need & $.341^{* *}[.227 * *-.336 * * *]$ & $.012[.010-.014]$ & $.004[.004-.007]$ \\
\hline \multicolumn{4}{|l|}{ Model 2} \\
\hline Race & $.023[-.061-(.014)]$ & $-.118[-.214-.082]$ & $.331[.321-.383]$ \\
\hline YASI Need & $.075[-.174-.164]$ & $.000[-.005-.007]$ & $.009[.006-.009]$ \\
\hline YASI Strength Total & $-.336[-.524 * * *-(-.231)]$ & $-.037[-.053-(-.023)]$ & $.020[.015-.021]$ \\
\hline Sex & $-.121[-.190-(-.165)]$ & $-.624[-.672-(-.421)]$ & $.331[.324-.389]$ \\
\hline \multicolumn{4}{|l|}{ Model 3} \\
\hline Race & $.001[-.085-(-.035)]$ & $-.196[-.297-.005]$ & $.337[.327-.390]$ \\
\hline YASI Need & $.081[-.175-.164]$ & $.001[-.008-.007]$ & $.009[.006-.009]$ \\
\hline YASI Strength Total & $-.401[-.6 * * *-(-.299)]$ & $-.004[-.061-(-.030)]$ & $.021[.017-.021]$ \\
\hline Sex & $-.318[-.393-(-.352)]$ & $-1.329[-1.387-(-1.108)]$ & $.676[.660-.771]$ \\
\hline Strength Total * Sex & $.221[.213-.240]$ & $.025[.023-.026]$ & $.021[.021-.024]$ \\
\hline
\end{tabular}

Note. Outcome variable is YLS/CMI 2.0 criminal history. $\beta$ is a standardized coefficient and $B$ is an unstandardized coefficient. SE $=$ standard error. For cases in which the estimates produced by the imputed datasets did not differ, the number is presented in place of the range. Model $1 R^{2}=.117$ (range of $.051-.112$ for multiple imputations). Model $1 F=4.98, p<.05$ (range of $2.85-6.61$ for multiple imputations). Model $2 R^{2}=.154$ (range of $.145-.157$ for multiple imputations). Model $2 F=3.31, p<.05$ (range of 4.35 4.80 for multiple imputations). Model $3 R^{2}=.166$ (range of $.155-.180$ for multiple imputations). Model $2 F=2.86, p<.05$ (range of 3.76- 4.49 for multiple imputations). ${ }^{*} p<.10 * * p<.05 * * * p<.001$. 
Specifically, the study did the following: 1) strengths and protective factors, as measured by the YLS/CMI 2.0 and YASI respectively, were profiled for males and females; 2) the YLS/CMI 2.0 strengths and YASI protective factors were correlated with each other where comparable for both males and females; 3 ) a gender moderated regression model was tested in order to determine if YLS/CMI 2.0 criminal history was post-dicted by YLS/CMI 2.0 strengths; and 4) a gender moderated regression model was tested in order to determine if YLS/CMI 2.0 criminal history was post-dicted by YASI protective factors.

\section{Inter-rater Reliability}

The YASI demonstrated acceptable inter-rater reliability in all but three domains: the social static risk domain, skills dynamic risk domain, and the attitudes dynamic protective domain. Low inter-rater reliability due to coding errors was ruled out as a cause. There are a number of reasons these domains had lower inter-rater reliability: 1) the domain only includes one or two items to be rated, therefore magnifying differences, 2) the domain was especially complex and subjective, causing marked differences in rating, or 3) the small sample of 21 magnified differences in rating. Also note that the same domains were on the lower end of acceptable ICCs in a report on YASI reliability in California's division of juvenile justice (Skeem, Hernandez, Kennealy, \& Rich, 2012). On the other hand, most of the risk domains of the YLS/CMI 2.0 had acceptable ICC's for the inter-rater reliability analysis, but all but one of the strength domains showed unacceptable reliability. To date, there are no other studies examining the psychometric properties of the strengths included in the YLS/CMI 2.0 measure. However, logic points to the lack of guidelines for assessors in the strength domains on the YLS/CMI 2.0 as the reason for poor inter-rater reliability in these domains.

\section{Profile of Strengths and Protective Factors.}

YLS/CMI 2.0. The YLS/CMI 2.0 has traditionally been one of the "gold standards" and 
is widely used for youth risk assessment. Often empirically ignored, the YLS/CMI 2.0 also has a strength component embedded within the tool that is to be used for case management purposes, much like the risk component. Chi-square results for the current sample of 59 female and 61 male justice involved youth indicate that none of the differences within domains were statistically significant. However, at face value, a higher percentage of males possessed YLS/CMI 2.0 strengths in the family, peers, substance abuse, leisure, and attitudes domains, but a higher percentage of females possessed strengths in the education and personality domains. In addition, the cumulative strength score (although the mean was slightly higher for males) was not significantly different between males and females. Cohen's $d$ for the difference between cumulative strengths scores among males and females indicated a small effect. The lack of adequate post-hoc power indicated that the lack of significant findings, at least for differences in cumulative strengths, may have been due to a small sample size, or that there was really no effect. At the very least, the profile of strengths on the YLS/CMI 2.0 for the current male and female sample demonstrates a trend where males are generally scored with a strength in the strengths domains more often than females.

YASI. Similar to the YLS/CMI 2.0 strength domains, males showed a general trend in scoring higher than females in most protective domains except for the attitudes static protective domain. Although the differences between males and females were not statistically significant in most protective domains on the YASI, males did score significantly higher than females in protection in the family dynamic protective and aggression dynamic protective domains and approached significance in the education dynamic protective domain. In addition, differences between males and females on the overall protective score approached significance. Examination of effect size and post-hoc power, indicate that either small sample size or lack of a genuine effect was likely the cause of a non-significant finding for male and female differences in the 
overall protective domain on the YASI.

Overall, the YASI seems to be highlighting more gender differences in strengths/protective factors than the YLS/CMI 2.0. However, with a larger sample size, gender differences on the YLS/CMI 2.0 could become more similar to those on the YASI as evidenced by low power. The second possibility is that the effects were simply not strong enough. The most striking finding is the general trend for males to be scoring higher in strengths and protective factors than females on both tools. Although very little, if any, literature focuses on the acute facets of strength differences between males and females in a young offender population, there may be a couple of reasons for the current findings. The current sample of males and females was matched on offence type and did not differ significantly on YLS/CMI 2.0 level of risk (with most being moderate or high risk offenders). In their article about effective interventions for delinquent girls, Hipwell and Loeber (2006) make reference to a theory where in the same span of time, girls require a significantly greater weight of negative life experiences or risk factors to get them to their offending "tipping point" than do males. In other words, it takes a lot more bad things to happen for a female to commit a crime, than for a male. This theory helps explain why our sample of female youth (although not significantly different than males), showed a general trend in having slightly higher risk than males did for the YLS/CMI 2.0 cumulative risk score. What this explicitly hints at is that the more risk one is at, the less strengths (as measured by the YASl) they also seem to have. In turn, the theory may also help to explain why the male sample showed a general trend in possessing more strengths than the female sample. When we matched the male and female samples for similar offences, keeping the tipping point theory in mind, the female sample hypothetically required more extreme negative situations than the males did to reach the same risk level or seriousness in offending. This line of reasoning also reiterates the need to account for context as so many feminist criminology scholars have argued is important 
for female offenders.

\section{Comparison of YLS/CMI 2.0 and YASI Strengths/Protective Factors}

Out of the eight pre-determined comparisons, three were found to correlate to a small degree for females and six were found to correlate for males. Of the domains of interest (i.e. where comparisons were logical), most YLS/CMI 2.0 and YASI strength/protective domains were at least weakly correlated with each other for males. With exception, the relationship between the YLS/CMI 2.0 education strength domain and the YASI education dynamic protective domain for males was very weak. The reason for a very weak correlation between the YLS/CMI 2.0 education and employment strength domain and the YASI education dynamic protective domain among males could be due to the non-congruence of the content of the domains. While the YLS/CMI 2.0 lumps employment with education, the YASI simply asks dynamic questions related to the youth's education and has a separate domain for employment related questions. Females did show a slightly stronger relationship between the YLS/CMI 2.0 education and employment strength domain and the YASI education domain, but it was not significantly stronger than that same relationship for males.

In addition, in the comparison of the YLS/CMI 2.0 peers strength domain and YASI peer dynamic protective domain, females demonstrated no relationship, which was significantly different from males who showed a strong relationship between the two domains. It is difficult to understand why some particular strength domains of the YLS/CMI 2.0 and YASI are not correlated. Difficulty in understanding results from the fact that the YLS/CMI 2.0 does not provide strict guidelines for scoring a youth with a strength, nor does it require the assessor to qualify in writing why they decided to score a youth with a strength. In other words, the YASI is very structured $\left(4^{\text {th }}\right.$ generation) in terms of protective domains, whereas the YLS/CMI 2.0 is, at best, marginal structured professional judgment where strengths are concerned. We can speculate 
that the reason males showed a strong relationship between the YASI and YLS/CMI 2.0 peer domains and the females showed no relationship has something to do with the qualitative differences between male and female peer relationships. Relational cultural theory can help to explain these differences; the theory emphasizes the importance of relationships in women's lives and by extension, girls' lives (Walker \& Rosen, 2004, Bloom \& Covington, 1998). Bloom and Covington (1998) point out that before 1976, when Jean Baker Miller discussed the development of women in her book: Toward a New Psychology of Women, theories in psychology described development as growing out of childlike dependence into mature independence. In other words, maturity was demonstrated by self-sufficiency, and autonomy. Miller (1976) suggested that this definition of development described the male experience rather than the female experience (Bloom \& Covington, 1998). In contrast, a woman's goal is to build connections with others through her life (Miller, 1976). Therefore, maturity for women is defined by connection as opposed to separation. The YASI protection items go into more detail than the YLS/CMI 2.0 items in gauging the quality of peer relationships as well as the quantity. The difference in the way the YASI and YLS/CMI 2.0 ask about strengths in the peer domain, may actually be reflecting fundamental differences in male and female peer relationships resulting in different correlations between the peer domains for males and females.

The attitudes strength domain of the YLS/CMI 2.0 and the YASI attitudes static protective domain were not correlated for males, but were strongly correlated for females. The profiles of gender differences in the attitudes domains of both tools did not indicate any gender differences. However, when looking back at the profile of attitudinal strengths for males and females, although no significant differences emerged, males evidenced a mildly higher rate of strengths in the attitudes domain on the YLS/CMI 2.0 than females and higher static protective attitude scores than females on the YASI. Consistent with this trend (although not statistically significant), the 
YLS/CMI 2.0 and YASI appear to be capturing strengths for males differently as evidenced by the correlations between the two domains. One possible explanation is inspired from the research of Jones (2011). In her sample of New York State youth, Jones found that the attitudes and skills domains on the New York State version of the YASI were the only domains that appeared to have genuine protective effects for males. More technically, higher protective scores in the attitudes domain were associated with a lower probability of recidivism for male youth only (not females) with higher overall risk scores than for youth with lower overall risk scores. Given that Jones (2011) found that the attitudes domain is acting as a genuine protective domain for males only, this could be the reason that the attitudes domains of the YLS/CMI 2.0 and the YASI are not correlated for males. Perhaps the YLS/CMI 2.0 is simply capturing promotive factors (factors that are negatively associated with recidivism) for males, but the YASI is capturing protective factors (factors that interact with recidivism differently at low and high levels of risk) in the attitudes domain.

Both males and females showed no correlation between the YLS/CMI 2.0 leisure strength domain and the YASI employment and free time static protective domain. In addition, the correlation between the YLS/CMI 2.0 leisure strengths domain and the YASI employment and free time dynamic protective domain was virtually absent for females, yet moderate for males. Although the content in both the YLS/CMI 2.0 leisure strength domain and the YASI static and dynamic protective employment and free time domains are related, the YASI combines employment and free time together, while the YLS/CMI 2.0 separates leisure from employment. Therefore, the absence of a relationship between the two domains could be due to the measurement of different constructs within the compared domains. Furthermore, the absence of a relationship between the dynamic employment/free time domain on the YASI and the education/employment domain on the YLS/CMI 2.0 for females, but a moderate one for males 
may be due to a difference in the construct being measured. There was a small effect for differences between males and females on the YASI dynamic protective employment and free time domain providing evidence that the YASI does not capture protective factors in the same way for males and females in this domain. Therefore, it cannot be expected that the correlations between the YASI dynamic protective employment and free time domain would be similarly correlated with any other domain for males and females.

The question remains, which assessment tool (YLS/CMI 2.0 or YASI) does a better job at capturing strengths and why are the domains incongruent in comparison with one another in some areas? Several explanations could be plausible: 1) the YASI takes into account the quality and quantity. For example, questions that gauge the type of attachment youth have to positively influencing peers, the number of positive adult relationships the youth has, and whether they are involved in positive community organizations helps to capture a more comprehensive 'snapshot' of a youth's strengths in the peers domain. On the other hand, the YLS/CMI 2.0 leaves it up to the individual assessor to decide whether the youth has a qualifying strength, which leaves very little room for empirical examination. 2) Perhaps one measure has 'it right' and the other does not, or both the YLS/CMI 2.0 and the YASI inadequately capture strengths and or protective factors. 3) Both the YASI and the YLS/CMI may both be measuring separate constructs very well, but those constructs are not highly correlated with each other. 4) The lack of correlations may demonstrate how much work remains to be done in the field-not only in terms of how to conceptualize and operationalize these strengths but how to incorporate them into risk assessment. In other words, both the YLS/CMI and the YASI purport that they measure protective factors, however the lack of strong correlations between the domains of the two tools suggests that they are not measuring the same construct, or at least not measuring it very well. Therefore, more of an effort needs to be made to distinguish between strengths, promotive, and 
protective factors, as well as to test the effects of these constructs on recidivism to see how they fit, or do not fit, into risk assessment tools.

\section{The Post-diction of YLS/CMI 2.0 Criminal History}

YLS/CMI 2.0 Strengths. To determine if YLS/CMI 2.0 strengths can post-dict YLS/CMI 2.0 criminal history and if strengths work differently in post-dicting criminal history for males and females, while taking into account the confounding variables of race and YLS/CMI 2.0 need, a hierarchical moderated regression model was used. The regression model included the covariates (race and YLS/CMI 2.0 need) the predictors (sex and YLS/CMI 2.0 strengths) and the dependent variable (YLS/CMI 2.0 Criminal History). Results indicated that the largest factor included in the regression model for post-dicting YLS/CMI 2.0 criminal history was the total YLS/CMI 2.0 need score that was comprised of seven domains (i.e., family, education/employment, peer relations, substance abuse, leisure/recreation, personality/behaviour, and attitudes). Not only did the interaction between gender and YLS/CMI 2.0 strengths not account for any significant variance in the model, but neither did gender or YLS/CMI 2.0 strengths, on their own, account for any significant amount of unique variance in the model. These findings indicate that perhaps the strength component of the YLS/CMI 2.0 would not aid in the prediction of recidivism for males or females over and above YLS/CMI 2.0 need. However, further analyses including recidivism data for the sample will need to be conducted once it is available.

YASI Protective Factors. To determine if YASI strengths can post-dict YLS/CMI 2.0 criminal history and if strengths work differently in post-dicting criminal history for males and females, while taking into account the confounding variables of race and YASI need, a hierarchical moderated regression model was used. The regression model included the covariates (race and YASI need), the predictors (sex and YASI strengths), and the dependent variable 
(YLS/CMI 2.0 criminal history). Results for the gender moderated regression using YASI protective factors to predict YLS/CMI 2.0 criminal history tell a slightly different story than the model using YLS/CMI 2.0 strengths as the predictor. The largest factor included in the regression model for post-dicting YLS/CMI 2.0 criminal history was actually the YASI protective factor score, indicating that perhaps YASI protective factors would actually add to the prediction of recidivism over and above YLS/CMI 2.0 need in future analyses once the data is available.

\section{Limitations}

There were the following limitations to the current study: 1) possibility of socially desirable responding, 2) small sample size, 3) unable to fully examine intersectionality, and 4) lack of recidivism data. For studies involving a face-to-face semi-structured interview, socially desirable responding is always a possibility, which could have potentially influenced an assessor's rating of a risk or protective item on one of the tools under investigation. However, in most cases the issue of socially desirable responding was overcome through thorough examination of criminal records and case files that either corroborated interviews or provided evidence against what a youth may have said. Review of criminal records and case files also helped to dampen any effect of self-report bias.

Another limitation to the current study is sample size. Due to the lower numbers of female youth in custody compared to male youth, a smaller subsample of males was taken from the entire sample in order to match them to females on offence history, bringing the sample numbers down considerably. However, results were reported in a very transparent way, taking into account effect size and post-hoc power where applicable. Despite the limited sample size it is encouraging that there were still some significant findings for the current study and that effect size analysis demonstrated some meaningful, albeit small gender differences. In addition, a 
smaller sample size limited the ability to look at the interaction between race and gender in the moderated regression analyses.

Finally, due to the two year time restriction for the current study, it does not include any findings with recidivism data. In turn, this prevented an answer to the question of whether the YLS/CMI or YASI contain any genuine protective factors; given more time, this is an excellent direction for future research.

\section{Conclusions and Future Directions}

This thesis takes a positive approach to the assessment of young male and female offenders by shifting the focus from risk and deficits to strengths. Every effort was made to investigate strengths, promotive, and protective factors in a sample of young offenders through a gender sensitive lens. The current study is the first to empirically examine the strength component of the YLS/CMI 2.0, paving the way for future research and modifications to the tool. In addition, the study investigates the protective component of the new YASI.

One of the major objectives of the study was to simply profile strengths, promotive, and protective factors in the sample, while looking for differences between males and females. The general trend, with some exceptions, was that males tended to mildly show slightly higher prevalence rates of strengths on both the YLS/CMI 2.0 and the YASI. Future research should aim to increase female offender sample sizes for comparison with male groups to see if these slight differences are actually statistically significant. These findings could have implications for modifying strengths-based treatment approaches to be more gender sensitive, as well as help to raise more awareness of the usefulness of strengths-based approaches.

The comparative analyses between the YLS/CMI 2.0 strengths and the YASI protective factors revealed that some domains that were expected to be highly correlated were not correlated at all. Furthermore, there were some cases where YLS/CMI 2.0 and YASI domains were not 
correlated for males, but were significantly correlated for females and vice versa. These findings suggest a few potential hypotheses: 1) the YLS/CMI 2.0 and YASI respectively, may not be consistent in the constructs they measure from domain to domain with in the tool (e.g. the YASI could be measuring promotive effects in the education domain, but genuine protective effects in the family domain); 2) the YLS/CMI 2.0 and YASI are not measuring the same construct between tools; 3) the YLS/CMI 2.0 and/or YASI may not be capturing strengths/promotive/protective factors equally as well for males and females in some domains; and 4) both the YLS/CMI 2.0 and the YASI may be measuring the same construct, each doing so inadequately. One discovery from this study was that the YLS/CMI 2.0 does not provide adequate instruction for the assessor in the qualifications of scoring a strength, nor does it provide a space for detailed information about why a rater decided to give a particular youth a strength. It is the present author's belief that modifications to the YLS/CMI 2.0 aimed at the strength component of the tool would prove useful in future research and perhaps in case management.

Finally, in the post-diction of YLS/CMI 2.0 criminal history, the overall YASI protective score was a significant predictor over and above YASI need. This finding lends more support to the possibility that the YASI measures a different construct than the YLS/CMI 2.0 in terms of protection for young offenders (given that the YLS/CMI 2.0 strengths were not a significant predictor in the model) and that it may hold more promise for strength-based approaches. Future research should make use of recidivism data when it is available for the current sample to provide insight into whether the YASI is actually measuring genuine protective factors.

This exploration of strengths/promotive/protective-based factors in justice involved youth at the very least, demonstrates the great need for more research directed at these approaches with samples of justice involved youth. The study also provides evidence that researchers should be 
mindful of gender differences when exploring strength/promotive/protective factors in youth. Future research should explore strength/promotive/protective factors that are not currently captured using risk assessment tools, but that may be more relevant for female youth. 


\section{References}

Andrews, D. A. (1980). Some experimental investigations of the principles of differential association through deliberate manipulations of the structure of service systems. American Sociological Review, 45, 448-462.

Andrews, D. A., \& Bonta, J. (2010). The psychology of criminal conduct (5 $5^{\text {th }}$ ed.). Cincinnati, $\mathrm{OH}$ : Anderson Publishing.

Andrews, D. A., Bonta, J., \& Wormith, S. J. (2004). The Level of Service/Case Management Inventory (LS/CMI). Toronto: Multi-Health Systems.

Andrews, D.A., Bonta, J., \& Wormith, S.J. (2006). The recent past and near future of risk and/or need assessment. Crime and Delinquency, 52, 7-27.

Andrews, D.A., \& Robinson, D. (1984). The level of supervision inventory: Second report. (Report to Research Services). Toronto: Ontario Ministry of Correctional Services. Andrews, D. A., Zinger, I., Hoge, R. D., Bonta, J., Gendreau, P., \& Cullen, F. T. (1990). Does correctional treatment work? A psychologically informed meta-analysis. Criminology, 28, 369-404. doi: 10.1111/j.1745-9125.1990.tb01330.x

Andrews, D.A. (1980). Some experimental investigations of the principles of differential association through deliberate manipulations of the structure of service systems. American Sociological Review, 45, 448-478.

Barnoski, R. (2003). Changes in Washington State's jurisdiction of juvenile offenders: Examining the impact. Olympia, WA: Washington State Institute of Public Policy. Retrieved from: http://www.wsipp.wa.gov/auth.asp?authid=3

Benda, B.B. (2005). Gender differences in life-course theory of recidivism: A survival analysis. International Journal of Offender Therapy and Comparative Criminology, 49, 325-342. 
Betchel, K. Lowenkamp, C.T. \& Latessa, E. (2007). Assessing the risk of reoffending for juvenile offenders using the Youth Level of Service/ Case Management Inventory. Journal of Offender Rehabilitation, 45, 85-108.

Bonta, J., \& Andrews, D.A. Risk-need-responsivity model for offender assessment and rehabilitation. Public Safety Canada. ISBN No.: 978-0-662-05049-0

Burgess, E.W. (1928). Factors determining success or failure on parole. In A. A. Bruce, A. J. Harno, E. W. Burgess, \& J. Landesco (Eds.), The working of the indeterminate sentence law and the parole system in Illinois (pp. 205-249).

Blanchette, K., \& Brown, S. L. (2006). The assessment and treatment of women offenders: An integrative perspective. Chichester, UK: John Wiley.

Blokland, A.A.J., \& Nieuwbeerta, P. (2005). The effects of life circumstances on longitudinal trajectories of offending. Criminology, 43, 1203-1240.

Bloom, B., \& Covingtion, S. (1998). Gender-specific programming for girls: what is it and why is it important? Paper presented at the $50^{\text {th }}$ Annual Meeting of the American Society of Criminology, November 11-14, 1998, Washington, D.C.

Bloom, B., Owen, B., \& Covington, S. (2005). Gender-responsive strategies: A Summary of research, practice, and guiding principles for women offenders (NIC Accession Number 02041). Washington, DC: U.S. Department of Justice, National Institute of Corrections.

Brown, S.L. \& Blanchette, K. (2008). Using a gender informed lens to advance theory and proactive for female offenders. Crime Scene: April 2008.

Catchpole, R., \&Gretton, M. (2003). The predictive validity of risk assessment with violent youth offenders: A one-year examination of criminal outcome. Criminal Justice and Behavior, 30, 688-708. 
Covington \& Bloom (2007). Gender responsive treatment and services in correctional settings. Women \& Therapy, 29 (3), 9-33.

Daly, K. (1992). Women's pathways to felony court: Feminist theories of lawbreaking and problems of representation. Review of Law and Women's Studies, 2(11), 11-52.

Deci, E.L., \& Ryan, R.M. (1985). Instrinsic motivation and self-determination in human behaviour. New York: Plenum Press

Dekovic, M. (1999). Risk and protective factors in the development of problem behaviour during adolescence. Journal of Youth and Adolescence, 28, (6), 667-685.

Dowden, C., \& Andrews, D. A. (1999). What works for female offenders: A meta-analytic review. Crime \& Delinquency, 45, 438-452. doi: 10.1177/0011128799045004002

Dowden, C., \& Andrews, D.A., (1999b). What works in young offender treatment: A metaanalysis. Forum on Corrections Research, 11, 21-24.

Dowden, C. \& Andrews, D.A. (2000). Effective correctional treatment and violent reoffending: A meta-analysis.

Edens, J., Campbell, J., \& Weir, J. (2007). Youth psychopathy and criminal recidivism: A metaanalysis of the Psychopathy Checklist measures. Law and Human Behavior, 31, 53-75.

Epstein, J.A., Botvin, G.J., Griffin, K.W., Diaz, T. (2001). Protective factors buffer effects of risk factors on alcohol use among inner-city youth. Journal of Child \& Adolescent Substance Abuse, 11 (1), 77-90.

Erikson, E., (1963). Childhood and Society (2 ${ }^{\text {nd }}$ ed.). New York: Basic Books.

Erikson, E., (1982). The life cycle completed. New York: Norton.

Fals-Stewart, W. (2003). The occurrence of partner physical aggression on days of alcohol consumption: A longitudinal diary study. Journal of Consulting and Clinical Psychology, $71,41-52$. 
Farrington, D. P. (2003). Developmental and life-course criminology: Key theoretical and empirical issues - The 2002 Sutherland Award Address. Criminology, 41, 221-255. doi: $10.1111 / \mathrm{j} .1745-9125.2003 . t b 00987 . x$

Fergus, S. \& Zimmerman, M.A. (2005). Adolescent resilience: a framework for understanding healthy development in the face of risk. Annual Review of Public Health, 26, 399-419.

Flores, A.W., Travis, L.F. \& Latessa, E.J. (2004). Case classification for juvenile corrections: An assessment of the Youth Level of Service and Case Management Iventory (YLS/CMI), final report.

Garmezy, N. Masten, A., \& Tellegen, A. (1984). The study of stress and competence in children: A building block for developmental psychopathology. Child Development, 55 (1), 97-111.

Garmezy, N. \& Masten, A. (1986). Stress, competence, and resilience: Common frontiers for therapist and psychopathologist. Behaviour Therapy, 17, 500-521.

Grove, W.M., Zald, D.H., Lebow, B.S., Snitz, B.E., \& Nelson, C. (2000). Clinical versus mechanical Prediction: A meta-analysis. Psychological Assessment, 12, 19-30.

Hannah-Moffat, K. (1999). Moral agent or actuarial subject: Risk and Canadian womens' imprisonment. Theoretical Criminology, 3 (1), $71-94$.

Hannah-Moffat, K. (2004). Gendering risk at what cost? Negotiations of gender and risk in Canadian women's prisons. Feminism \& Psychology, 14(2), 243-249.

Hannah-Moffat, K., \& Shaw, M. (2001). Taking risks: Incorporating gender and culture into classification and assessment of federally sentenced women in Canada. Ottawa, Ontario, Canada: Status of Women Canada.

Hart, J. L., O'Toole, S. K., Price-Sharps, J. L., \& Shaffer, T. W. (2007). The risk and protective factors of violent juvenile offending: An examination of gender differences. Youth Violence and Juvenile Justice, 5, 367-384. doi: 10.1177/1541204006297367 
Hipwell \& Loeber. (2006). Do we know we know which interventions are effective for disruptive and delinquent girls. Clinical Child and Family Psychology Review, 9, 221-255.

Hoffman, P.B., \& Beck, J.L. (1974). Parole decision making: A salient factor score. Journal of Criminal Justice, 2, 195-206.

Hoge, R. D., \& Andrews, D. A. (2011). The Youth Level of Service/Case Management Inventory 2.0 (YLS/CMI 2.0): User's manual. Toronto, ON: Multi-Health Systems.

Holtfreter, K., \& Cupp, R. (2007). Gender and risk assessment: The empirical status of the LSI-R for women. Journal of Contemporary Criminal Justice, 23, 363-382. doi: $10.1177 / 1043986207309436$

Holtfreter, K., Reisig, D., \& Morash, M. (2004). Poverty, state capital, and recidivism among women offenders. Criminology \& Public Policy, 3, 185-208. doi: 10.1111/j.17459133.2004.tb00035.x

Hussong, A.M., Curran, P.J., Moffitt, T.E., Caspi, A., \& Carrig, M.M. (2004). Substance abuse hinders desistance in young adults' antisocial behavior. Development and Psychopathology,16, 1029-1046.

Jahoda, M. (1958). Current concepts of positive mental health. New York: Basic Books.

Jessor, R. (1991). Risk behavior in adolescence: A psychosocial framework for understanding and action. Journal of Adolescent Health, 12, 597-605.

Jones, N,J. (2011). Merging theoretical frameworks to inform risk assessment for the young female offender. Unpublished doctoral dissertation, Carleton University, Ottawa, Ontario, Canada.

Kashdan, T. B., Mishra, A., Breen, W. E., \& Froh, J. J. (2009). Gender differences in gratitude: Examining appraisals, narratives, the willingness to express emotions, and changes in 
psychological needs. Journal of Personality, 77, 691-730.

Kimonis, E.R., Frick, P.J., Skeem, J.L., Marsee, M.A., Cruise, K., Munoz, L. C., Aucoin, K.J., \& Morris, A.S. (2008). Assessing callous-unemotional traits in adolescent offenders: Validation of the Inventory of Callous-Unemotional Traits. International Journal of Law and Psychiatry, 31, 2008.

Kong, R., \& AuCoin, K. (2008). Female offenders in Canada (Report No. 85-002-XIE, Vol. 28, no. 1). Ottawa, ON: Statistics Canada.

Lipsey, M. (2009). The primary factors that characterize effective interventions with juvenile offenders: A meta-analytic overview. Victims and Offenders, 4 (2), 124-147. doi: $10.1080 / 15564880802612573$.

Luthar, S.S. \& Cicchetti, D. (2000). The construct of resilience: Implications for interventions and social policies. Development and Psychopathology, 12, 857-885.

Luthar, Cicchetti, and Becker (2000). The construct of resilience: A critical evaluation and guidelines for future work. Child Development, 71(3), 543-562.

Marczyk, G.R., Heilbrun, K., Lander, T., \& DeMatteo, D. (2005). Juvenile decertification: Developing a model for classification and prediction. Criminal Justice and Behaviour, 32, 278-301.

Marshall, J. Egan, V., English, M., \& Jones, R.M. (2006). The relative validity of psychopathy versus risk/needs-based assessments in the prediction of adolescent offending behaviour. Legal and Criminological Psychology, 11, 197-210.

Maruna, S. (2001). Making good: How ex-convicts reform and rebuild their lives. Washington, DC: American Psychological Association.

Maruna, S., LeBel, T.P., Mitchell, N., \& Naples, M. (2004). Pygmalion in the reintegration process: Desistance from crime through the looking glass. Psychology, Crime \& Law, 10, 
271-281

Maslow, A.H., (1954). Motivation and personality. New York: Harper \& Row.

Maslow, A.H., (1962). Toward a psychology of being. Princeton, NJ: Van Nostrand.

Masten, A. S. (2001). Ordinary magic: Resilience processes in development. American Psychologist, 56, 227-238. doi: 10.1037/0003-066X.56.3.227

Masten, Garmezy, Tellegen, Pellegrini, Larkin, \& Larsen (1988). Competence and stress in school children: The moderating effects of individual and family qualities. Journal of child Pscyhology, 29 (6), 745-764.

Maume, M.O., Ousey, G.C., \& Beaver, K. (2005). Cutting the grass: A reexamination of the link between marital attachment, delinquent peers and desistance from marijuana use. Journal of Quantitative Criminology, 21, 27-53.

Morton, G. M., \& Leslie, L. A. (2005). The adolescent female delinquent. Journal of Feminist Family Therapy, 17, 17-50. doi: 10.1300/J086v17n01_02

Motiuk, L. L., Bonta, J., \& Andrews, D. A. (1990). Dynamic predictive criterion validity in offender assessment. Paper presented at the Canadian Psychological Association Annual Convention, Ottawa.

Nuffield, J. (1982). Parole decision-making in Canada: Research towards decision guidelines. Ottawa, ON: Solicitor General of Canada.

Orbis Partners. (2007a). Long-term validation of the Youth Assessment and Screening Instrument (YASI) in New York State juvenile probation. Retrieved April 25, 2011, from http://dpca.state.ny.us/pdfs/nyltyasifullreport20feb08.pdf

Orbis Partners. (2007c). Youth Assessment and Screening Instrument: Girls (YASI-G). Ottawa, Ontario, Canada.

Park, N., Peterson, C., \& Seligman, M.E. (2004). Strengths of character and well-being. Journal 
of Social and Clinical Psychology, 23, 603-619.

Park, N., Peterson, C., \& Seligman, M.E. (2005a). Character strengths in forty nations and fifty states. Unpublished manuscript, University of Rhode Island.

Park, N., Peterson, C., \& Seligman, M.E. (2005b). Strengths of character and well-being among youth. Unpublished manuscript, University of Rhode Island.

Pellegrini, masten, garmezy \& Ferrarese (1987). Correlates of social and academic competence in middle childhood. Journal of child psychology, 28 (5), 699-714.

Peterson, C., \& Seligman, M.E., (2004). Character strengths and virtues. New York: Oxford University Press.

Raynor, P. (2007) Risk and need assessment in British probation: The contribution of the LSI-R. Psychology, Crime and Law, 13, 125-138.

Raynor, P., Kynch, J., Roberts, C., \& Merrington, S. (2000). Risk and need assessment in probation services: an evalution. Home Office Research Study No. 2011. London, England: Home Office.

Rettinger, L. J., \& Andrews, D. A. (2010). General risk and need, gender specificity, and the recidivism of female offenders. Criminal Justice and Behavior, 37, 29-46. doi: $10.1177 / 0093854809349438$

Rogers, C. (1951). On becoming a person. Cambridge, MA: Riverside Press.

Rutter, M., Giller, H., \& Hagell, A. (1998). Antisocial behavior by young children. New York: Cambridge University Press.

Ryff, C.D., \& Singer, B. (1996). Psychological well-being: Meaning, measurement and implications for psychotherapy research. Psychotherapy and Psychosomatics, 65, 14-23

Sampson, R.J., \& Laub, J.H. (2005). A life-course view of the development of crime. Annals of the American Academy of Political and Social Science, 602, 12-45. 
Schwalbe, C. (2007). Risk assessment for juvenile offenders: A meta-analysis. Law and Human Behavior, 31, 449-462.

Schwalbe, C. (2008). A meta-analysis of juvenile justice risk assessment instruments: Predictive validity by gender. Criminal Justice and Behaviour, 35 (11), 1367-1381. doi:

$1177 / 0093854808324377$.

Seligman, M. \& Csikszentmihalyi, M. (2000). Positive psychology: An introduction. American Psychologist, 55(1), 5-14.

Seligman, M. E. P. (2002). Positive psychology, positive prevention, and positive therapy. In C.

R. Snyder \& S. J. Lopez (Eds.), Handbook of positive psychology (pp. 3-9). New York: Oxford University Press.

Serin, R. \& Lloyd, C. (2009). Examining the process of offender change: the transition to crime desistance. Psychology, Crime, \& Law, 15 (4), 347-364.

Simourd, L., \& Andrews, D. A. (1994). Correlates of delinquency: A look at gender differences. Forum on Corrections Research, 6(1), 26-31. Retrieved July 19, 2011 from http://www.csc-scc.gc.ca/text/pblct/forum/e061/061g_e.pdf

Skeem, J., Hernandez, I., Kenealy, P., \& Rich, J. (2012). CA-YASI reliability: How adequately do staff in California's Division of Juvenile Justice rate youth's risk of recidivism? University of California, Irvine. Unpublished report.

Sorbello, L, Eccleston, L., Ward, T., \& Jones, R. (2002). Treatment needs of female offenders: A review. Australian Psychologist, 37, 198-205. doi: 10.1080/00050060210001706876

Tabachnick, B. G., \& Fidell, L. S. (2012). Using multivariate statistics $\left(6^{\text {th }}\right.$ ed.). Boston, MA: Pearson.

Tweed, R.G., Bhatt, G., Dooley, S., \& Spindler, A. (2011). Youth violence and positive psychology: Research potential through integration. Canadian Psychology, 52 (2), 
111-121. doi:10.1037/a0020695

Uggen, C. (1999). Ex-offenders and the conformist alternative: A job quality model of work and crime. Social Problems, 46, 127-151.

Valliant, G.E. (1977). Adaptation to life. Boston: Little, Brown.

Van Voorhis, P. (2012). On behalf of women offenders: Women's place in the science of evidenced based practice. American Society of Criminology, 11 (2), 111-145.

Vieira, T., Skilling, T., Peterson-Badali, M. (2009) Matching court-ordered services with treatment needs: Predicting treatment success with young offenders. Criminal Justice and Behavior, 36, 385-401. doi:10.1177/0093854808331249

Viljoen, J.,Elkovitch, N., Scalora, M., \& Ullman, D. (2009). Assessing risk for violence in adolescents who have sexually offended: An examination of the predictive validity of the ERASOR, YLS/CMI, PCL-YV. Criminal Justice and Behavior, 36, 981-1000.

Walker, M., \& Rosen, W.B. (2004). How connections heal: Stories from Relational-Cultural Therapy. New York, NY: Guilford Press.

Ward, T. (2002). The management of risk and the design of good lives. Australian Psychologist, $37(3), 172-179$.

Ward, T. \& Gannon, T. (2006). Rehabilitation, etiology, and self-regulation: The comprehensive good lives model of treatment for sexual offenders. Aggression and Violent Behaviour, 11 , 77-94. doi:10.1016/j.avb.2005.06.00.

Welsh, J.L., Schmidt, F., McKinnon, L., Chattha, H.K., \& Myers, J.R. (2008). A comparative study of adolescent risk assessments: Predictive and incremental validity. Assessment, 15, 104-115.

Werner, E.E. (1996). Vulnerable but invincible: High risk children from birth to adulthood. European Child and Adolescent Psychiatry, 5, 47-51. (Original work published 1982) 
Werner, E.E., and Smith, R.S. (1992). Overcoming the odds: high risk children from birth to adulthood (Cornell University Press, Ithaca, NY).

Whitney, S.D., Renner, L.M. \& Herronkohl, T.I. (2010). Gender differences in risk and promotive classifications associated with adolescent delinquency. The Journal of Genetic Psychology, 171 (2), 116-138.

Yates, T. M., \& Masten, A. S. (2004). Fostering the future: Resilience theory and the practice of positive psychology. In P. A. Linley \& S. Joseph (Eds.), Positive psychology in practice (pp. 521-539). Hoboken, NJ: Wile 
Appendix $A$

Risk Classification Thresholds for YASI Pre-Screen and Items by Domain Pre-Screen Risk Cutoffs for Girls Cutoffs for Boys

$\begin{array}{lcc}\text { Low } & 0-25 & 0-15 \\ \text { Medium } & 26-52 & 16-38 \\ \text { High } & \geq 53 & \geq 39\end{array}$

\section{Pre-Screen Total $=$ Legal History Risk Score + Social History Risk Score}

\section{Legal History (14 items)}

Previous police contacts for delinquent/criminal offenses (A01SR)

Age at first police contact for delinquent/criminal offenses (A02SR)

Number of police contacts (A03SR)

Police contacts for felony offenses: (Category 1 offenses) (A04SR)

Transfers to adult court (A05SR)

Number of weapon offenses (A06SR)

Police contact for offenses against another person (A07SR)

Police contacts for felony (Category 1) offenses against another person (A08SR)

Number of placements with children or youth services (A09SR)

Number of times admitted to remand (A10SR)

Number of times admitted to custody - either open or secure (A11SR)

Escapes (A12SR)

Failure to appear in court (A13SR)

Number of Petitions for violations of probation or supervision (A14SR)

\section{Social History (17 items)}

Parental authority (P06SR)

Circumstances of family members living in household (PI5SR)

Run away or kicked out (B01aSR + B01bSR)

Court finding of child neglect (B02SR)

Compliance with parental rules (B03DR and B03DP)

Circumstances of family members living in the household (B041DR and B042DR)

Current school enrollment status (C01DR)

School attendance in previous 3 months (C02DR)

School conduct in previous 3 months (C03DR)

Academic performance in previous 3 months (C04DR)

Associates the youth spends his or her time with

(D01DR)

Substance abuse (E01DR)

Mental Health Problems (F01SR and F01DR)

Homicidal ideation (F02SR)

Suicidal Ideation (F03SR)

Sexual aggression (F04SR) 
Types of violence (G01SR)

Accepts responsibility for delinquent behavior (H01DR)

Consequential thinking skills (I01DR) 
An Exploration of Strengths, Promotive and Protective Factors

Appendix $B$

Risk Classification Thresholds for YASI Items by Domain

Legal History Risk Cutoffs for Girls $\quad$ Cutoffs for Boys

\begin{tabular}{lcc}
\hline Low & $0-4$ & $0-1$ \\
Medium & $5-17$ & $2-12$ \\
High & $\geq 18$ & $\geq 13$ \\
\hline
\end{tabular}

Static Risk Classification Thresholds for YASI Family Domain

\begin{tabular}{|c|c|c|}
\hline Family Static Risk & Cutoffs for Girls & Cutoffs for Boys \\
\hline Zero & 0 & 0 \\
\hline Low & 1 & 1 \\
\hline Medium & $2-3$ & $2-3$ \\
\hline High & $\geq 4$ & $\geq 4$ \\
\hline
\end{tabular}

Dynamic Risk Classification Thresholds for YASI Family Domain

\begin{tabular}{llcc}
\hline \multirow{2}{*}{ Fisk } & Cutoffs for Girls & Cutoffs for Boys \\
\hline Zero & & 0 \\
Low & 0 & $1-3$ \\
Medium & $1-16$ & $4-16$ \\
High & $17-29$ & $\geq 17$ \\
\hline
\end{tabular}

Static Risk Classification Thresholds for YASI School Domain

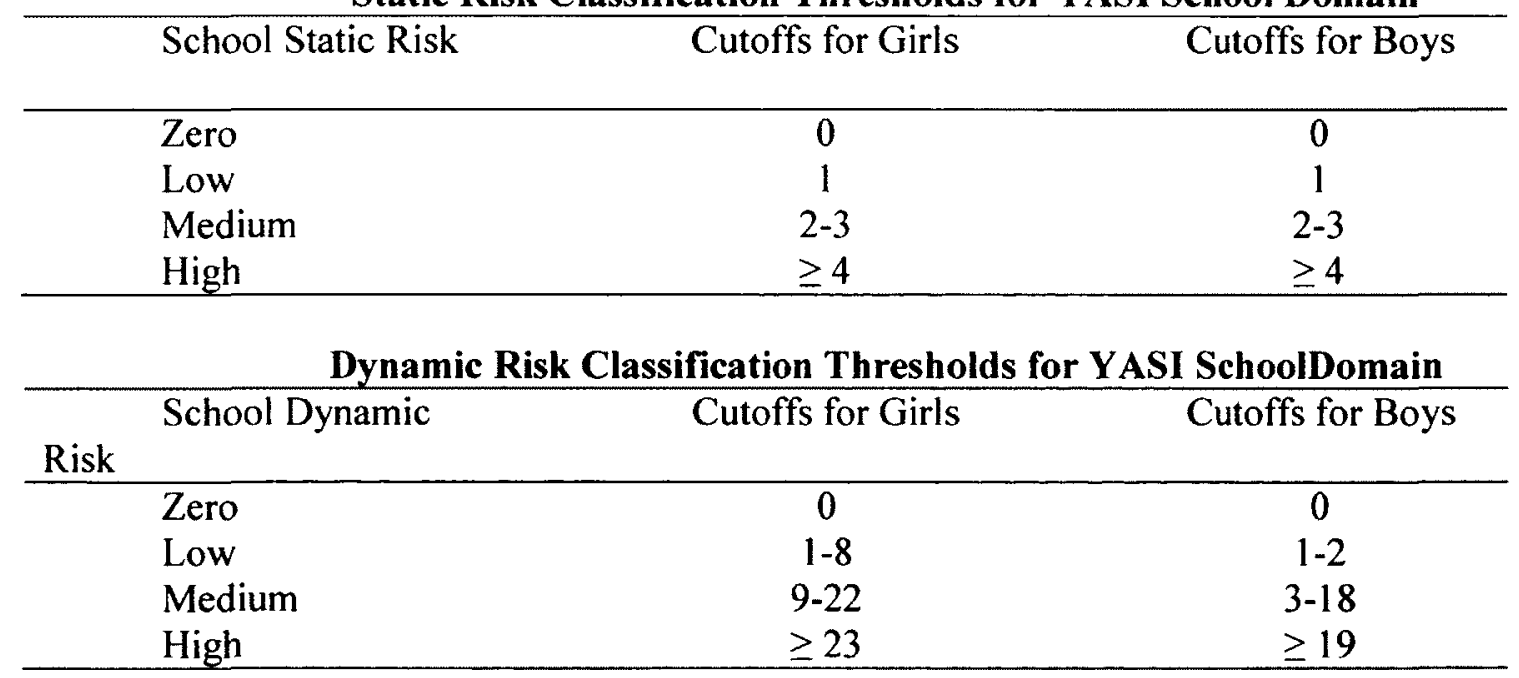


Static Risk Classification Thresholds for YASI Community and Peers Domain

Community and Cutoffs for Girls Cutoffs for Boys

Peers Dynamic Risk

\begin{tabular}{lcc} 
Zero & 0 & 0 \\
Low & 1 & 1 \\
Medium & $2-3$ & $2-3$ \\
High & $\geq 4$ & $\geq 4$ \\
\hline
\end{tabular}

Dynamic Risk Classification Thresholds for YASI Community and Peers Domain

\begin{tabular}{|c|c|c|c|}
\hline Risk & Family Dynamic & Cutoffs for Girls & Cutoffs for Boys \\
\hline & Zero & 0 & 0 \\
\hline & Low & $1-9$ & $1-7$ \\
\hline & Medium & $10-23$ & $8-16$ \\
\hline & High & $\geq 24$ & $\geq 17$ \\
\hline
\end{tabular}

Static Risk Classification Thresholds for YASI Substance Abuse Domain Substance Abuse Cutoffs for Girls Cutoffs for Boys Static Risk

\begin{tabular}{lcc} 
Zero & -- & - \\
Low & 1 & 1 \\
Medium & 2 & $2-12$ \\
High & $\geq 3$ & $\geq 13$ \\
\hline
\end{tabular}

Dynamic Risk Classification Thresholds for YASI Substance Abuse Domain Substance Abuse Cutoffs for Girls Cutoffs for Boys Dynamic Risk

\begin{tabular}{lcc}
\hline Zero & 0 & 0 \\
Low & $1-4$ & $1-6$ \\
Medium & $5-19$ & $7-11$ \\
High & $\geq 20$ & $\geq 12$ \\
\hline
\end{tabular}

Static Risk Classification Thresholds for YASI Mental Health Domain

\begin{tabular}{lcc}
\hline Mental Health Risk & Cutoffs for Girls & Cutoffs for Boys \\
\hline Zero & 0 & 0 \\
Low & 1 & 1 \\
Medium & $2-5$ & $2-5$ \\
High & $\geq 6$ & $\geq 6$ \\
\hline
\end{tabular}


Dynamic Risk Classification Thresholds for YASI Aggression Domain

\begin{tabular}{ccc}
\hline $\begin{array}{c}\text { Aggression } \\
\text { Dynamic Risk }\end{array}$ & Cutoffs for Girls & Cutoffs for Boys \\
\hline Zero & 0 & 0 \\
Low & $1-10$ & $1-3$ \\
Medium & $11-16$ & $4-13$ \\
High & $\geq 17$ & $\geq 14$ \\
\hline
\end{tabular}

Static Risk Classification Thresholds for YASI Attitudes Domain

\begin{tabular}{lcc}
\hline Attitudes Static Risk & Cutoffs for Girls & Cutoffs for Boys \\
\hline Zero & 0 & 0 \\
Low & -- & -- \\
Medium & 1 & 1 \\
High & $\geq 2$ & $\geq 2$ \\
\hline
\end{tabular}

Dynamic Risk Classification Thresholds for YASI Attitudes Domain

\begin{tabular}{|c|c|c|c|}
\hline Risk & Attitudes Dynamic & Cutoffs for Girls & Cutoffs for Boys \\
\hline & Zero & 0 & 0 \\
\hline & Low & $1-9$ & $1-6$ \\
\hline & Medium & $10-26$ & $7-26$ \\
\hline & High & $\geq 27$ & $\geq 27$ \\
\hline
\end{tabular}

Dynamic Risk Classification Thresholds for YASI Skills Domain

\begin{tabular}{|c|c|c|c|}
\hline Risk & Skills Dynamic & Cutoffs for Girls & Cutoffs for Boys \\
\hline & Zero & 0 & 0 \\
\hline & Low & $1-15$ & $1-7$ \\
\hline & Medium & $16-28$ & $8-25$ \\
\hline & High & $\geq 29$ & $\geq 26$ \\
\hline
\end{tabular}

Static Risk Classification Thresholds for YASI Employment and Leisure Domain

\begin{tabular}{llcc}
\hline & Employment Static & Cutoffs for Girls & Cutoffs for Boys \\
Risk & & -- & -- \\
\hline Zero & 1 & 1 \\
Low & $\geq 2$ & $\geq 2$ \\
Medium & -- & -- \\
High & & \\
\hline
\end{tabular}


An Exploration of Strengths, Promotive and Protective Factors

Dynamic Risk Classification Thresholds for YASI Employment and Leisure Domain Employment Static

Cutoffs for Girls

Cutoffs for Boys

Risk

\begin{tabular}{lcc} 
Zero & 0 & 0 \\
Low & $1-4$ & $1-2$ \\
Medium & $5-6$ & $3-5$ \\
High & $\geq 7$ & $\geq 7$ \\
\hline
\end{tabular}

Static Risk Classification Thresholds for YASI Full Assessment

\begin{tabular}{llc}
\hline Employment Static & Cutoffs for Girls & Cutoffs for Boys \\
\hline Zero & & -- \\
Low & $-\overline{-11}$ & $0-11$ \\
Medium & $12-17$ & $12-17$ \\
High & $\geq 18$ & $\geq 18$ \\
\hline
\end{tabular}

Dynamic Risk Classification Thresholds for YASI Full Assessment

\begin{tabular}{|c|c|c|c|}
\hline Risk & Employment Static & Cutoffs for Girls & Cutoffs for Boys \\
\hline & Zero & -- & -- \\
\hline & Low & $0-78$ & $0-33$ \\
\hline & Moderate low & $79-102$ & $34-56$ \\
\hline & Moderate & $103-124$ & $57-91$ \\
\hline & Moderate High & $125-157$ & $92-121$ \\
\hline & High & $158-175$ & $122-142$ \\
\hline & Very High & $\geq 176$ & $\geq 143$ \\
\hline
\end{tabular}

Dynamic Protective Classification Thresholds for YASI Family Domain Family Static Risk Cutoffs for Girls $\quad$ Cutoffs for Boys

\begin{tabular}{lcc}
\hline Zero & 0 & 0 \\
Low & $1-4$ & $1-5$ \\
Medium & $5-9$ & $6-14$ \\
High & $\geq 10$ & $\geq 15$ \\
\hline
\end{tabular}

Dynamic Protective Classification Thresholds for YASI School Domain School Static Risk Cutoffs for Girls Cutoffs for Boys

\begin{tabular}{lcc}
\hline Zero & 0 & 0 \\
Low & $1-6$ & $1-5$ \\
Medium & $7-10$ & $6-11$ \\
High & $\geq 11$ & $\geq 12$ \\
\hline
\end{tabular}


An Exploration of Strengths, Promotive and Protective Factors

Dynamic Protective Classification Thresholds for YASI Community and Peers Domain

\begin{tabular}{clcc}
\hline \multirow{2}{*}{ Fisk } & Camily Dynamic & Cutoffs for Girls & Cutoffs for Boys \\
\hline Zero & 0 & 0 \\
Low & $1-5$ & $1-2$ \\
Medium & $6-11$ & $3-10$ \\
High & $\geq 12$ & $\geq 11$ \\
\hline
\end{tabular}

Dynamic Protective Classification Thresholds for YASI Aggression Domain Aggression Cutoffs for Girls Cutoffs for Boys Dynamic Risk

\begin{tabular}{lcc} 
Zero & 0 & 0 \\
Low & 1 & 1 \\
Medium & 2 & $2-3$ \\
High & $\geq 3$ & $\geq 4$ \\
\hline
\end{tabular}

Static Protective Classification Thresholds for YASI Attitudes Domain Attitudes Static Risk Cutoffs for Girls Cutoffs for Boys

\begin{tabular}{lcc}
\hline Zero & 0 & 0 \\
Low & -- & - \\
Medium & 1 & 1 \\
High & $\geq 2$ & $\geq 2$ \\
\hline
\end{tabular}

Dynamic Protective Classification Thresholds for YASI Attitudes Domain

\begin{tabular}{cccc}
\hline & Attitudes Dynamic & Cutoffs for Girls & Cutoffs for Boys \\
Risk & & & 0 \\
\hline Zero & 0 & $1-5$ \\
Low & $1-9$ & $6-16$ \\
Medium & $10-26$ & $\geq 17$ \\
High & $\geq 27$ & \\
\hline
\end{tabular}

Dynamic Protective Classification Thresholds for YASI Skills Domain

\begin{tabular}{cccc}
\hline \multirow{2}{*}{ Risk } & Cutoffs for Girls & Cutoffs for Boys \\
\hline Zero & 0 & 0 \\
Low & $1-8$ & $1-8$ \\
Medium & $9-15$ & $9-15$ \\
High & $\geq 16$ & $\geq 16$ \\
\hline
\end{tabular}


An Exploration of Strengths, Promotive and Protective Factors

Static Protective Classification Thresholds for YASI Employment and Leisure Domain

\begin{tabular}{llcc}
\hline \multirow{2}{*}{ Risk } & Employment Static & Cutoffs for Girls & Cutoffs for Boys \\
\hline Zero & 0 & 0 \\
Low & 1 & 1 \\
Medium & $2-3$ & $2-3$ \\
High & $\geq 4$ & $\geq 4$ \\
\hline
\end{tabular}

Dynamic Protective Classification Thresholds for YASI Employment and Leisure Domain

\begin{tabular}{clcc}
\hline \multirow{2}{*}{ Risk } & Employment Static & Cutoffs for Girls & Cutoffs for Boys \\
\hline Zero & 0 & 0 \\
Low & 1 & 1 \\
Medium & -- & -- \\
High & $\geq 2$ & $\geq 2$ \\
\hline
\end{tabular}

Static Protective Classification Thresholds for YASI Full Assessment

\begin{tabular}{llcc}
\hline & Employment Static & Cutoffs for Girls & Cutoffs for Boys \\
Risk & & 0 & 0 \\
Zero & 1 & 1 \\
Low & $2-3$ & $2-3$ \\
Moderate & $\geq 4$ & $\geq 4$ \\
High & $\geq 4$ \\
\hline
\end{tabular}

Dynamic Protective Classification Thresholds for YASI Full Assessment

\begin{tabular}{lcc} 
Employment Static & Cutoffs for Girls & Cutoffs for Boys \\
Low & $0-1$ & $0-4$ \\
Moderate low & $2-6$ & $5-10$ \\
Moderate & $7-13$ & $11-23$ \\
Moderate High & $14-23$ & $24-44$ \\
High & $24-33$ & $45-67$ \\
Very High & $\geq 34$ & $\geq 68$ \\
\hline
\end{tabular}


An Exploration of Strengths, Promotive and Protective Factors

Overall Protective Classification Thresholds for YASI Full Assessment

\begin{tabular}{ccc}
\hline Employment Static & Cutoffs for Girls & Cutoffs for Boys \\
\hline Low & & $0-4$ \\
Moderate low & $0-1$ & $5-10$ \\
Moderate & $2-6$ & $11-23$ \\
Moderate High & $7-13$ & $24-44$ \\
High & $14-23$ & $45-67$ \\
Very High & $24-33$ & $\geq 68$ \\
\hline
\end{tabular}




\section{Appendix $C$}

\section{YASI Full Assessment: Items by Domain}

\section{Legal History (14 items)}

Previous police contacts for delinquent/criminal offenses (A0ISR)

Age at first police contact for delinquent/criminal offenses (A02SR)

Number of police contacts (A03SR)

Police contacts for felony offenses: (Category 1 offenses) (A04SR)

Transfers to adult court (A05SR)

Number of weapon offenses (A06SR)

Police contact for offenses against another person (A07SR)

Police contacts for felony (Category 1) offenses against another person (A08SR)

Number of placements with children or youth services (A09SR)

Number of times admitted to remand (A10SR)

Number of times admitted to custody-either open or secure (A11SR)

Escapes (A12SR)

Failure to appear in court (A13SR)

Number of Petitions for violations of probation or supervision (A 14SR)

\section{Family History (16 items)}

Times kicked out of home (B01 aSR)

Times run away $(\mathrm{B} 0 \mathrm{lbSR})$

Has there ever been a family court finding of neglect (B02SR)

Compliance with parental rules (B03DR and B03DP)

Circumstances of family members who are living in the household (B04DR) 
Historic problems of family members in the environment the youth was raised (B05SR) Youth's current living arrangements (B06a) (not scored)

Parental/custodial supervision (B07DR and B07 DP)

Appropriate consequences for bad behaviour (B08DR and B08DP)

Appropriate rewards for good behaviour (B09DR and B09DP)

Parental attitude towards youth's maladaptive behaviour (B10DR)

Support network for family; extended family and friends who can provide additional support (B1 1DP)

Family member(s) youth feels close to or has a good relationship with (B12DP)

Family provides opportunities for youth to participate in family activities (B13DP)

Family provides opportunities for youth to learn, grow, and succeed (B14DP)

Parental love, caring, and support for youth (B15DR and B15DR)

Level of conflict in the home (B16DR)

\section{School (13 items)}

Highest grade completed (C00i) (Not scored)

Current school enrollment status (C01) (Not scored)

Youth's attendance in last three months of school (C02DR and C02DP)

Youth's conduct in the last three months of school (C03DR and C03DP)

Youth's academic performance in last three months of school (C04DR and C04DP)

Youth's current school conduct (C05DR)

Youth's current academic performance (C06) (Not scored)

Special education status (C07SR)

Youth believes receiving an education is important to him/her (C08DR and C08DP) 
Youth believes school provides a supportive and encouraging environment (C09DR and C09DP)

Out-of-school suspensions in last two years (Cl0aSR)

In-school suspensions in last two years (C10bSR)

Expulsions since first grade (C10cSR)

Age at first suspension (C11SR)

Youth's involvement in school activities during the most recent school year (C12DR and C12DP)

Teachers/staff/coaches youth likes or feels comfortable talking to (C12DP)

\section{Peers and Community (8 items)}

Associates the youth spends his or her time with (D01DR and D01DP)

Attachment to positive influencing peers (D02DR and D02DP)

Admiration/emulation of high risk delinquent peers (D03DR and D03DP)

Number of months youth has been associating with delinquent peers (D04SR)

Amount of free time youth spends with negatively influencing peers (D05DR and

D05DP)

Strength of negative peer influence (D06DR and D06DP)

Number of existing positive adult relationships in community (D07DP)

Prosocial community ties (D08DP)

\section{Alcohol and Drugs (3 items)}

Alcohol/drug use (E0la) (Not scored)

(Drug) Times used in the last three months (E01cDR) 
(Drug) Disrupts functioning (E01dDR)

(Drug) Contributes to behavior (E01eDR)

(Drug) Age at first use (E01fSR)

(Drug) Attempts to cut back (E01g) (Not scored)

Youth is receptive to participate in alcohol/drug treatment (E02DR)

Previous alcohol/drug treatment (E03SR)

\author{
Mental Health (6 items) \\ Mental health problems (F01a) (Not scored) \\ Type of mental health problem (F01bSR and F01bDR) \\ Homicidal ideation (F02SR) \\ Suicidal ideation (F03SR) \\ Sexual aggression (F04) (Not scored) \\ Victimization (F06SR)
}

\title{
Aggression (and Violence) (5 items)
}

Violence (G01SR)

Hostile interpretation of actions and intentions of others in a common nonconfrontational setting (G02DR and G02DP)

Tolerance for frustration (G03DR and G03DP)

Belief in use of physical violence or verbal aggression (G04DR)

Belief in use of verbal aggression to solve a disagreement or conflict (G05DR) 


\section{Attitudes (8 items)}

Accepts responsibility for delinquent behaviour (H0IDR and H01DP)

Understands impact of behaviour on others (H02DR and H02DP)

Willingness to make amends (H03DR and H03DP)

Optimism (H04DR and H04DP)

Attitude when engaged in delinquent behaviour (e.g., hyper, nervous, etc.) (H05SR and H05SP)

Law-abiding attitudes (H06DR and H06DP)

Respect for authority figures (H07DR and Ho7DP)

Readiness for change (H08DR and H08DP)

\section{Skills ( 7 items)}

Consequential thinking skills (I01DR and I01DP)

Social perspective-taking skills (I02DR and I02DP)

Problem-solving skills (I03DR and I03DP)

Impulse control (I04DR and I04DP)

Loss of control over maladaptive behaviour (I05DR and I05DP)

Interpersonal skills (I06DR and I06DP)

Goal-setting skills (I07DR and I07DP)

\section{Employment and Free Time (8 items)}

History of employment (J01SR, J01SP, and J01DP)

Total number of times employed (J02SP)

Number of weeks of longest period of employment (J03SP and J03DP) 
An Exploration of Strengths, Promotive and Protective Factors

Positive relationships with employers or adult co-workers (J04DP)

Structured recreational activities (J05DR and J05DP)

Unstructured recreational activities (J06DR and J06DP)

Challenging/exciting hobbies (J07DR and J07DP)

Decline/increase in interest in positive leisure pursuits (J08DR and J08DP) 


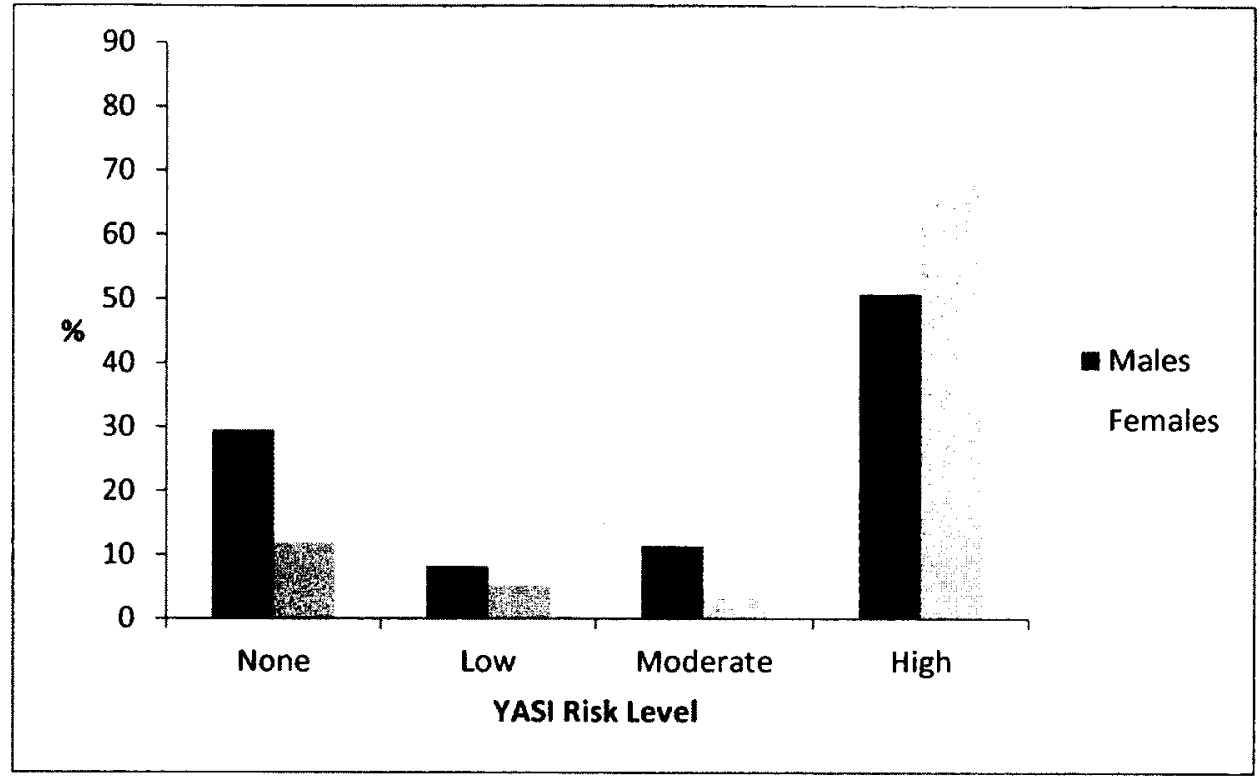

Figure 1. Distribution of Youth for YASI Family Domain Static Risk Levels

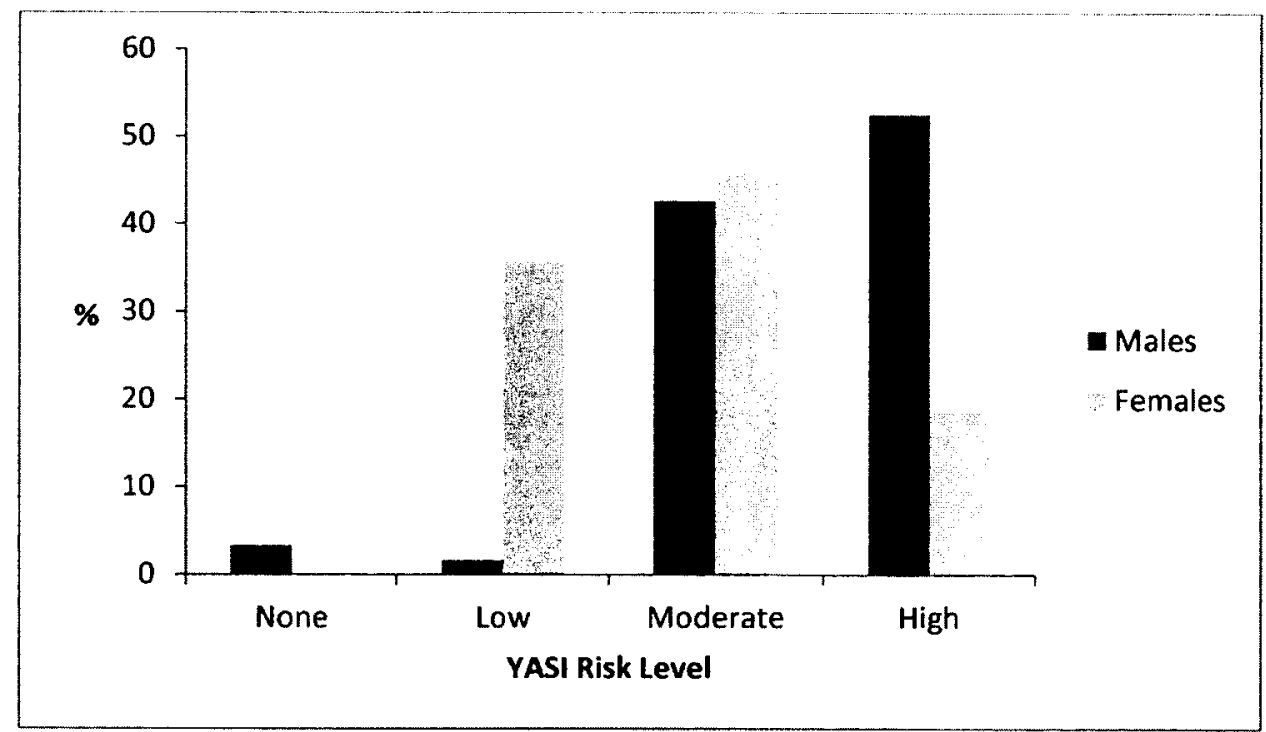

Figure 2. Distribution of Youth for YASI Family Domain Dynamic Risk Levels 


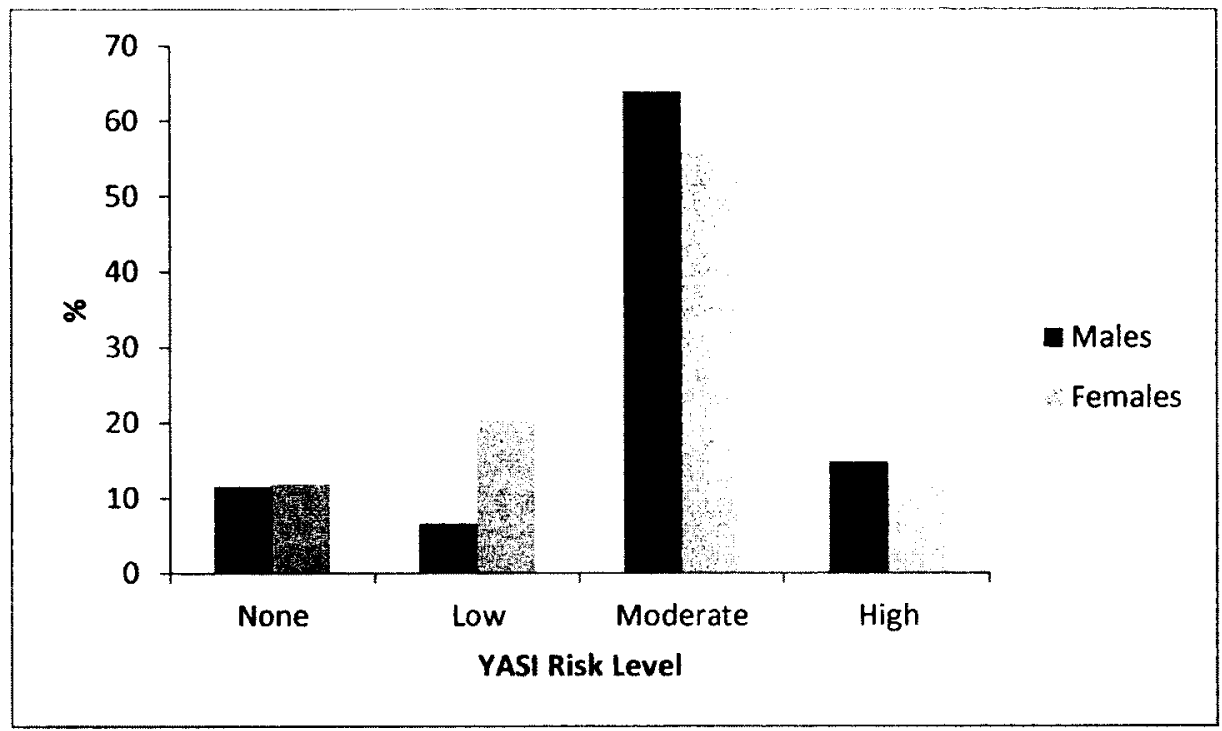

Figure 3. Distribution of Youth for YASI Education Domain Static Risk Levels

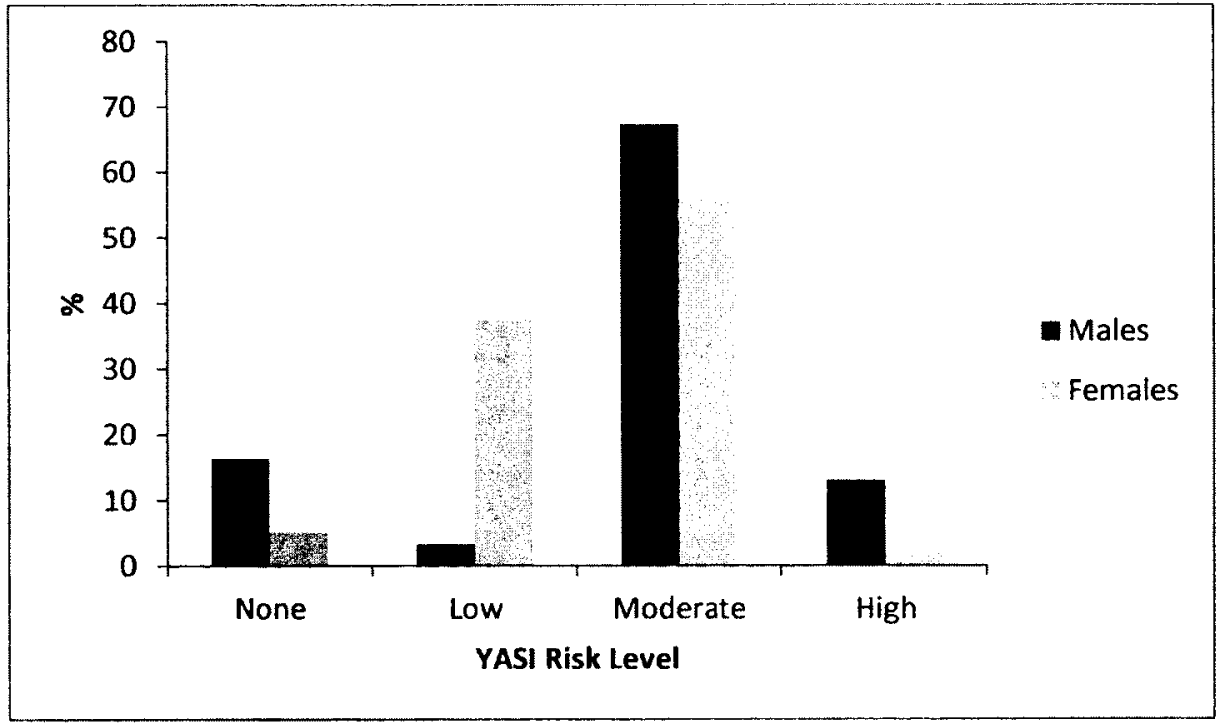

Figure 4. Distribution of Youth for YASI Education Domain Dynamic Risk Levels 
An Exploration of Strengths, Promotive and Protective Factors

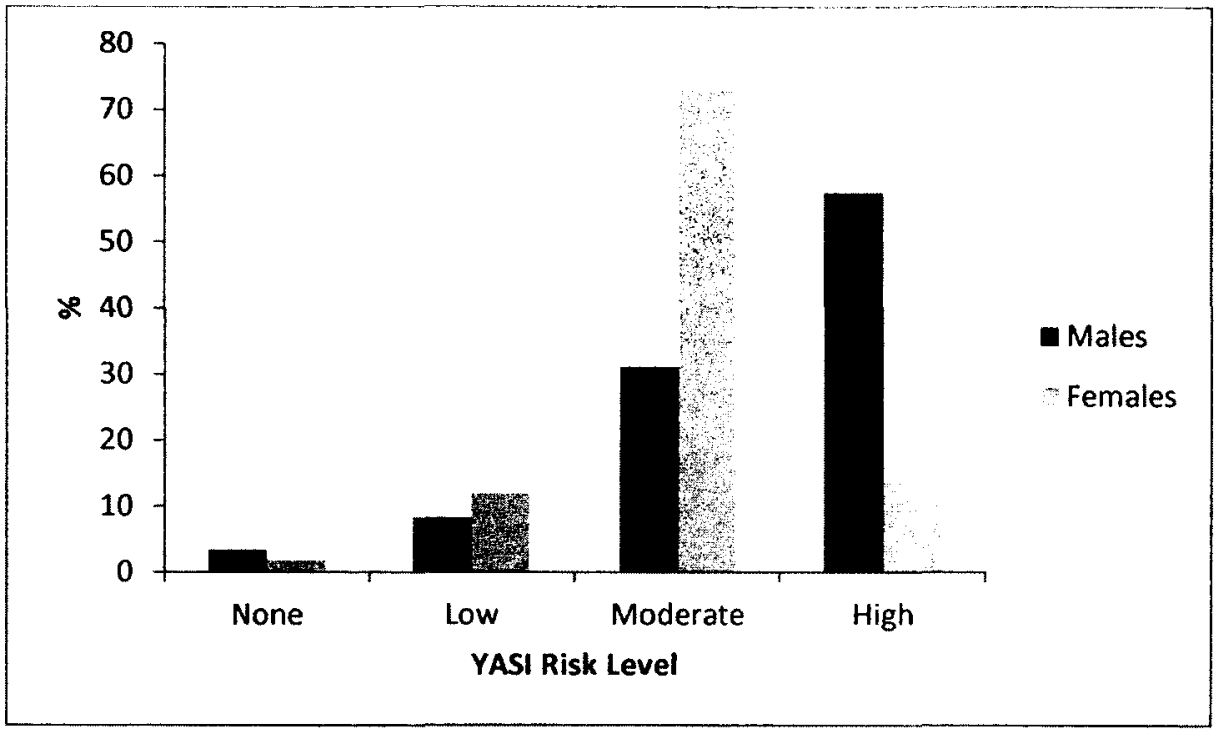

Figure 5. Distribution of Youth for YASI Social Domain Dynamic Risk Levels

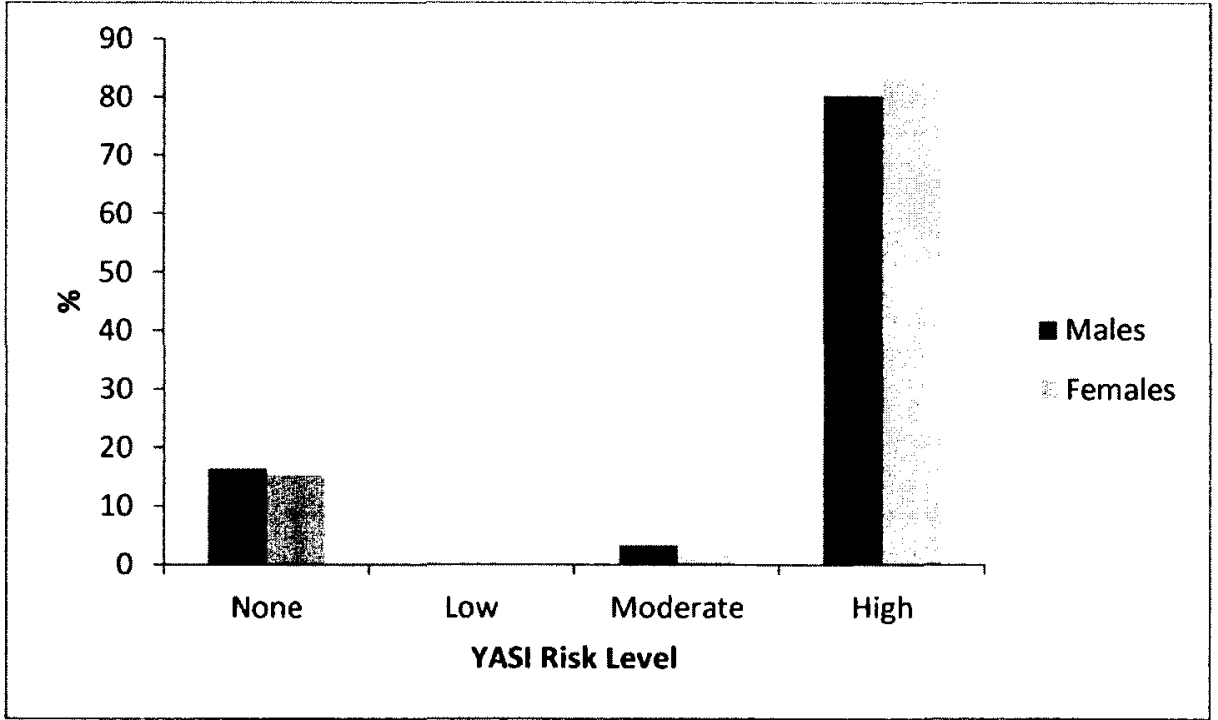

Figure 6. Distribution of Youth for YASI Social Domain Static Risk Levels 


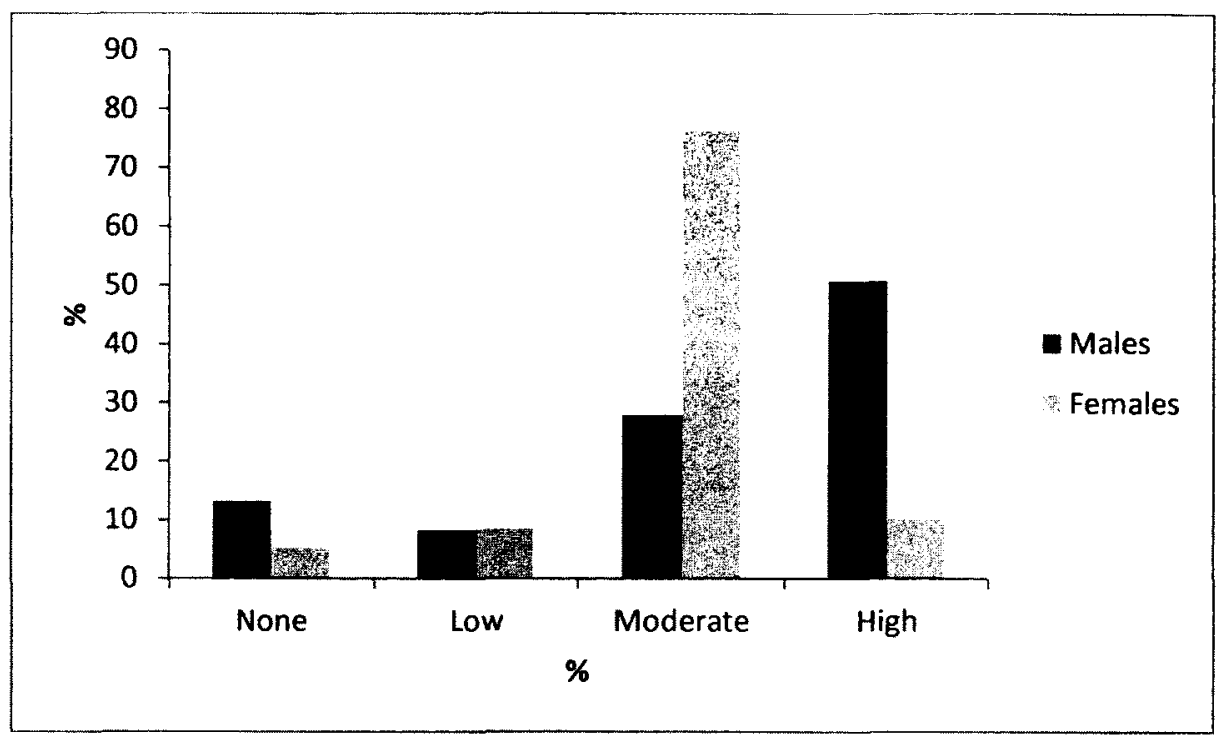

Figure 7. Distribution of Youth for YASI Substance Abuse Domain Dynamic Risk Levels

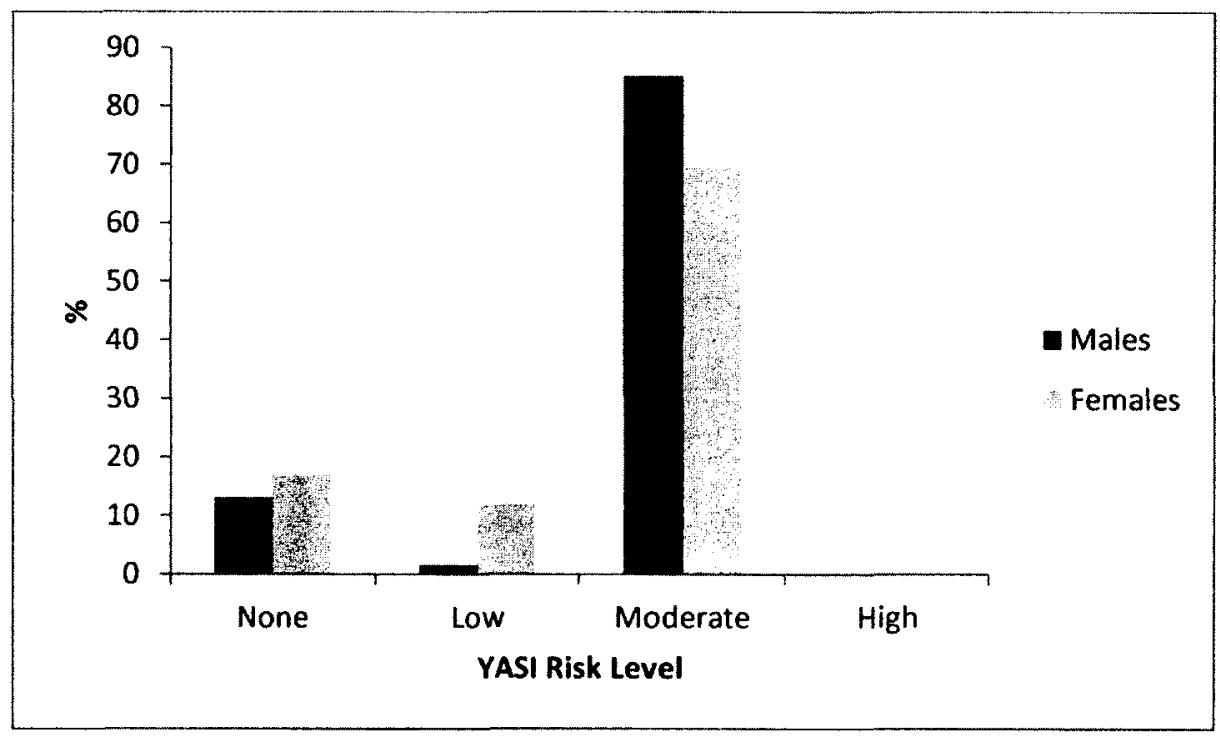

Figure 8. Distribution of Youth for YASI Substance Abuse Domain Static Risk Levels 


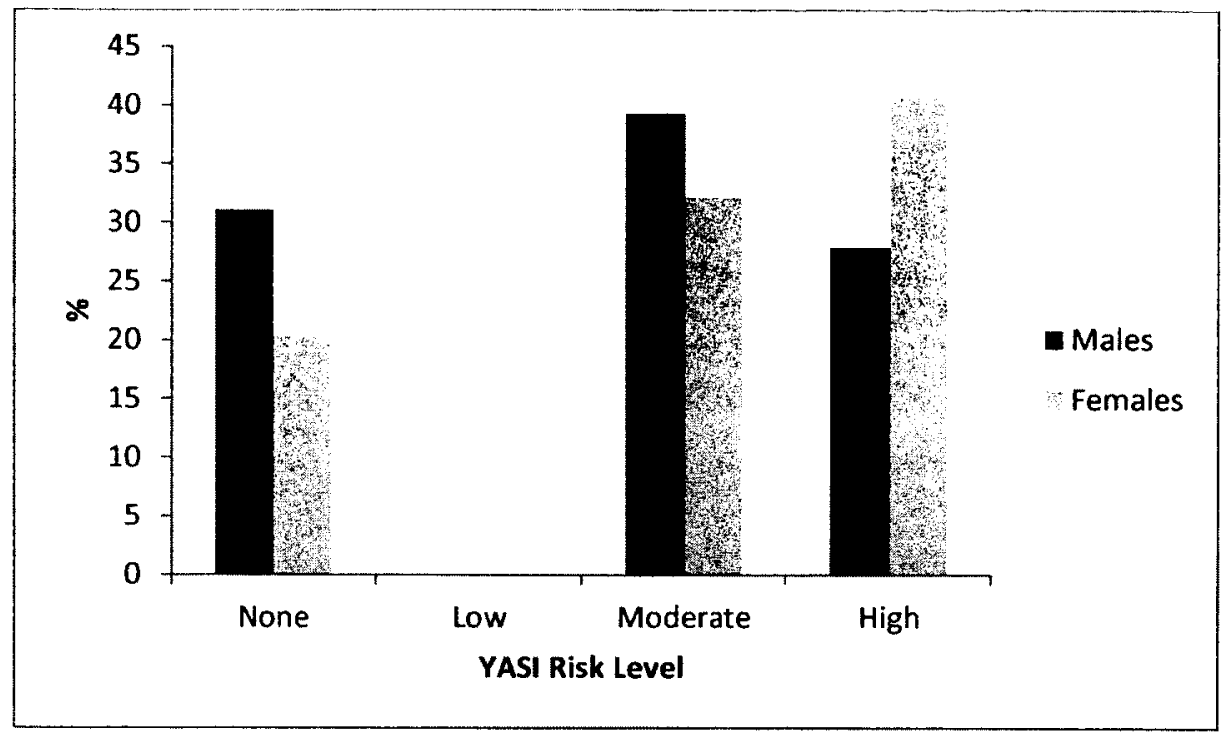

Figure 9. Distribution of Youth for YASI Mental Health Domain Risk Levels

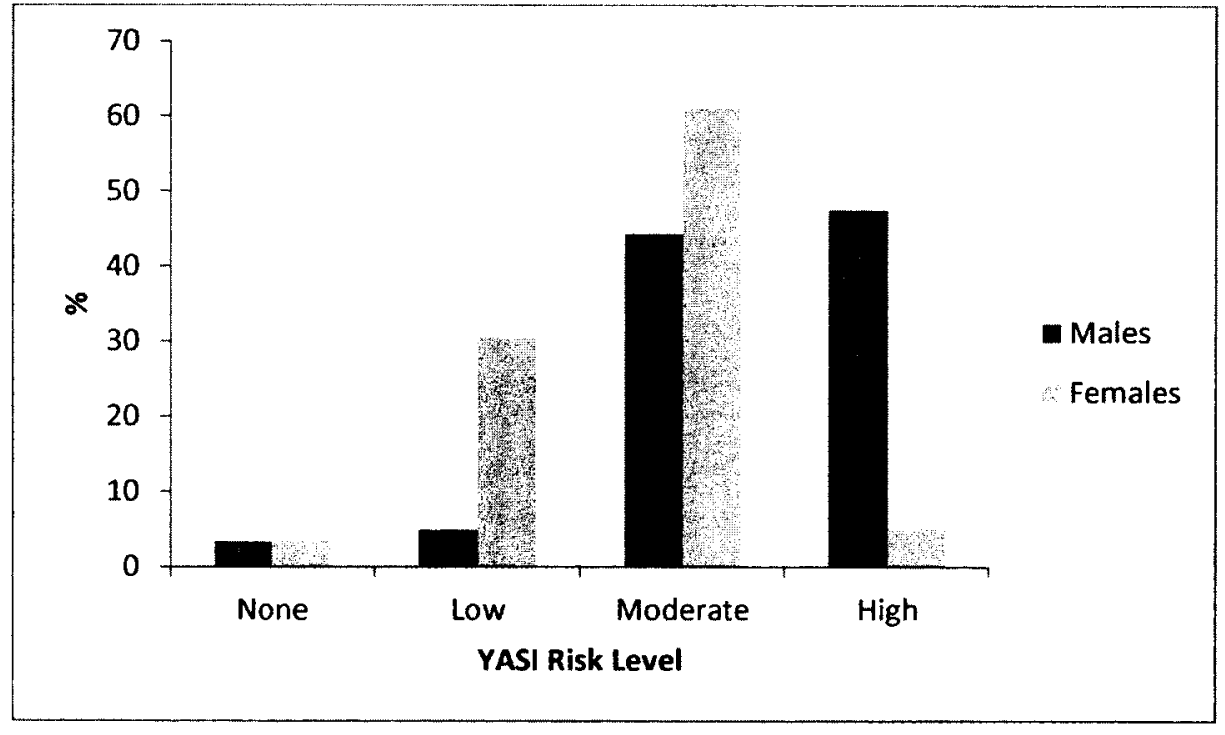

Figure 10. Distribution of Youth for YASI Aggression Domain Dynamic Risk Levels 


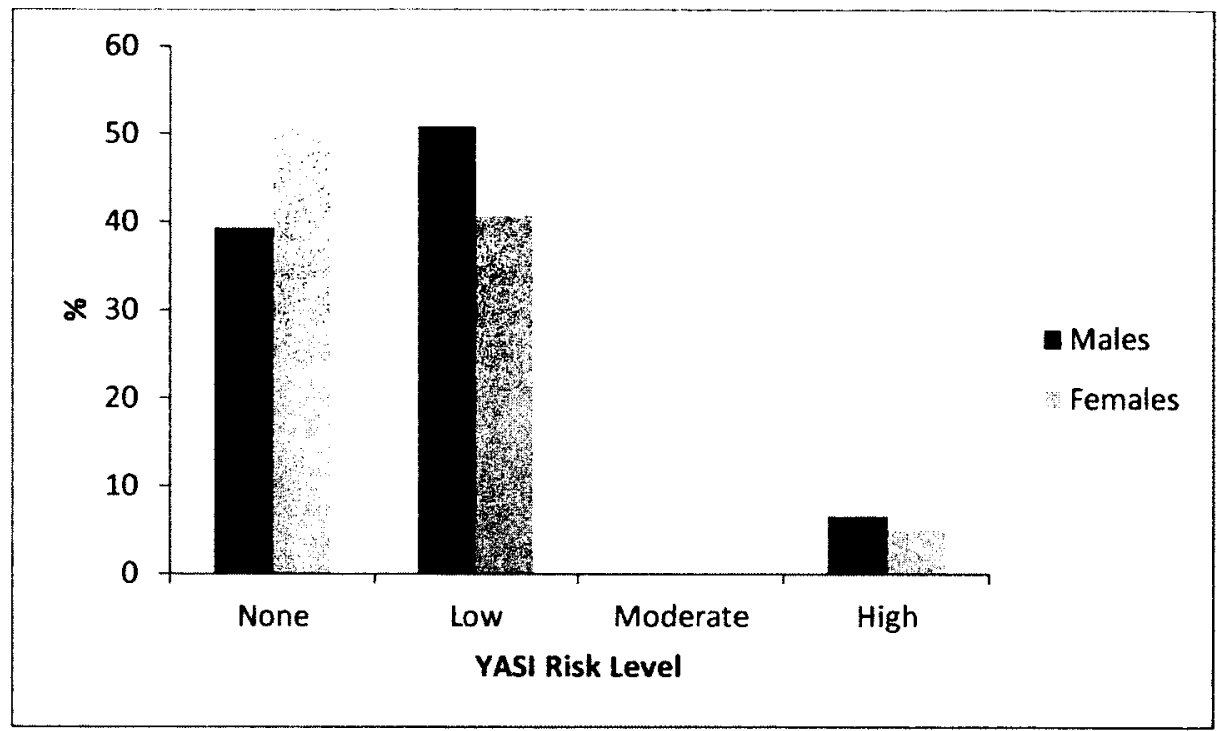

Figure 11. Distribution of Youth for YASI Attitude Domain Static Risk Levels

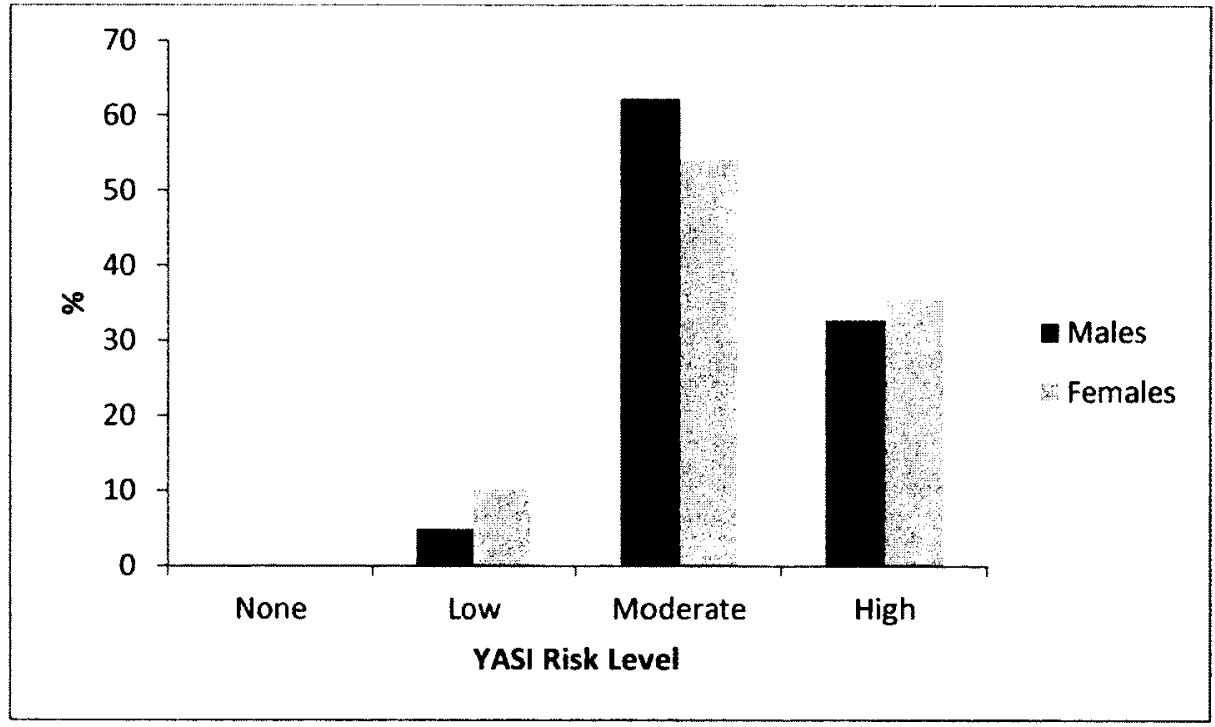

Figure 12. Distribution of Youth for YASI Attitude Domain Dynamic Risk Levels 


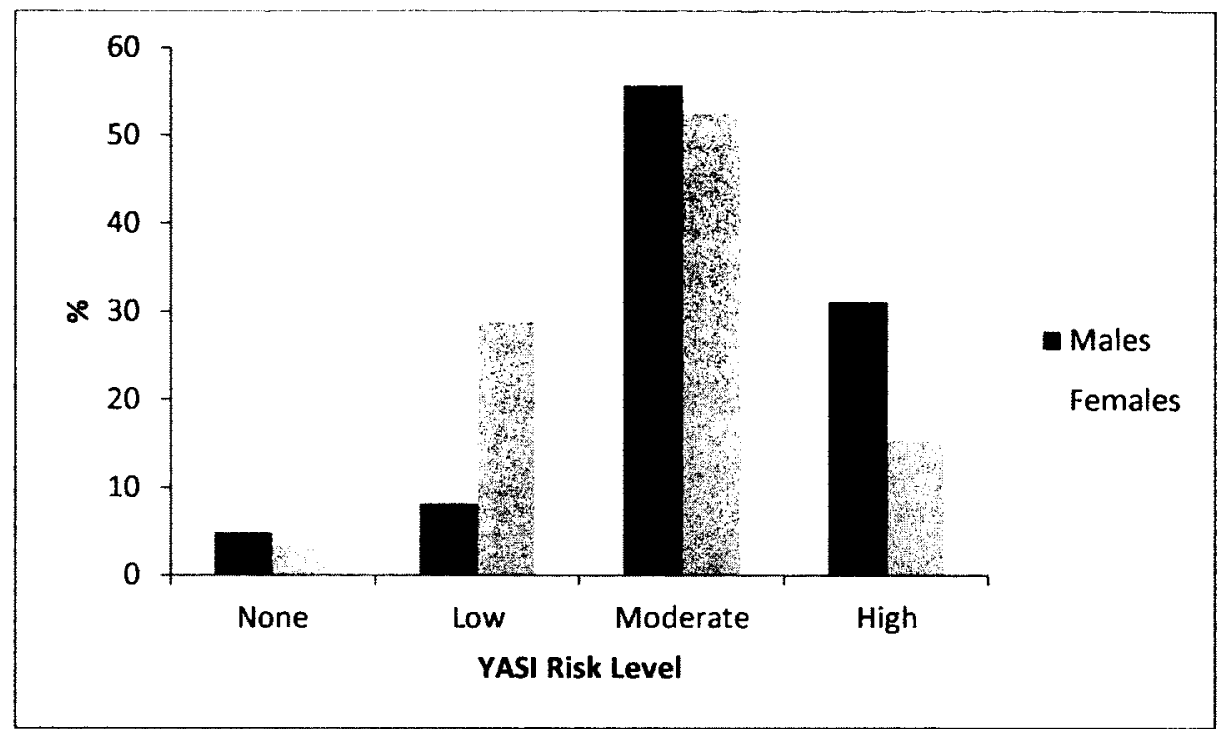

Figure 13. Distribution of Youth for YASI Skills Domain Dynamic Risk Levels

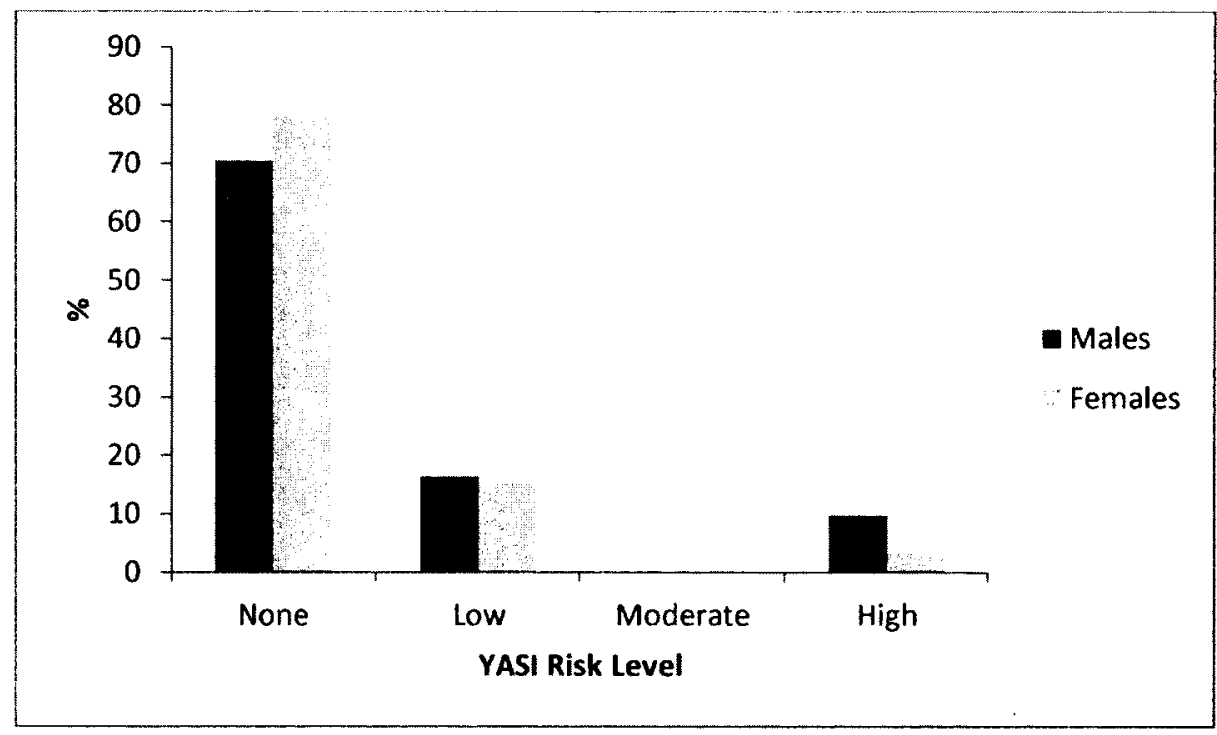

Figure 14. Distribution of Youth for YASI Employment Domain Static Risk Levels 
An Exploration of Strengths, Promotive and Protective Factors

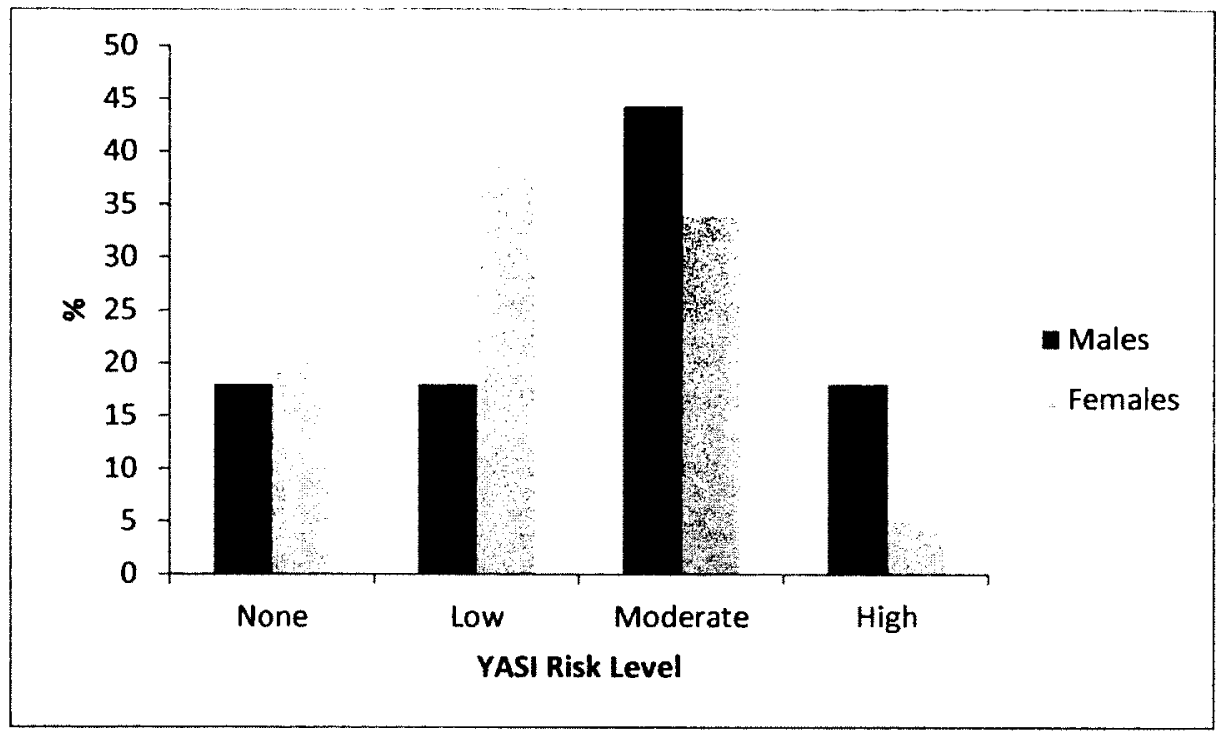

Figure 15. Distribution of Youth for YASI Employment Domain Dynamic Risk Levels

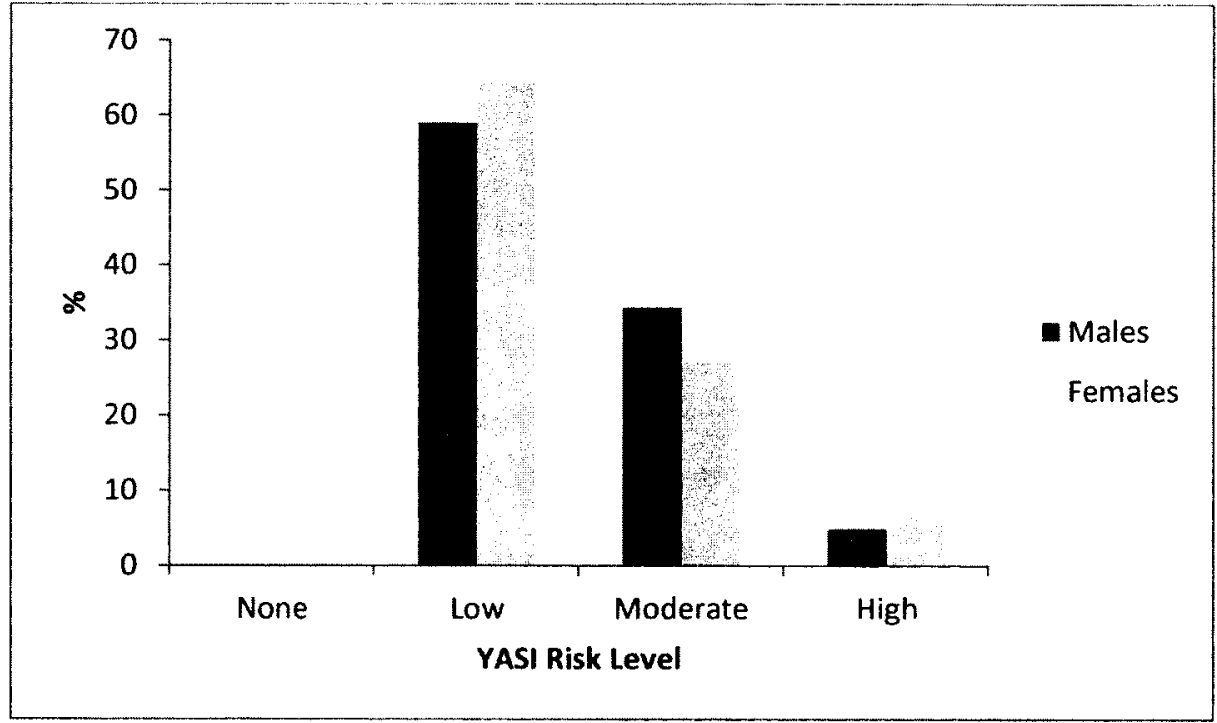

Figure 16. Distribution of Youth for YASI Famiily Domain Dynamic Protective Levels 


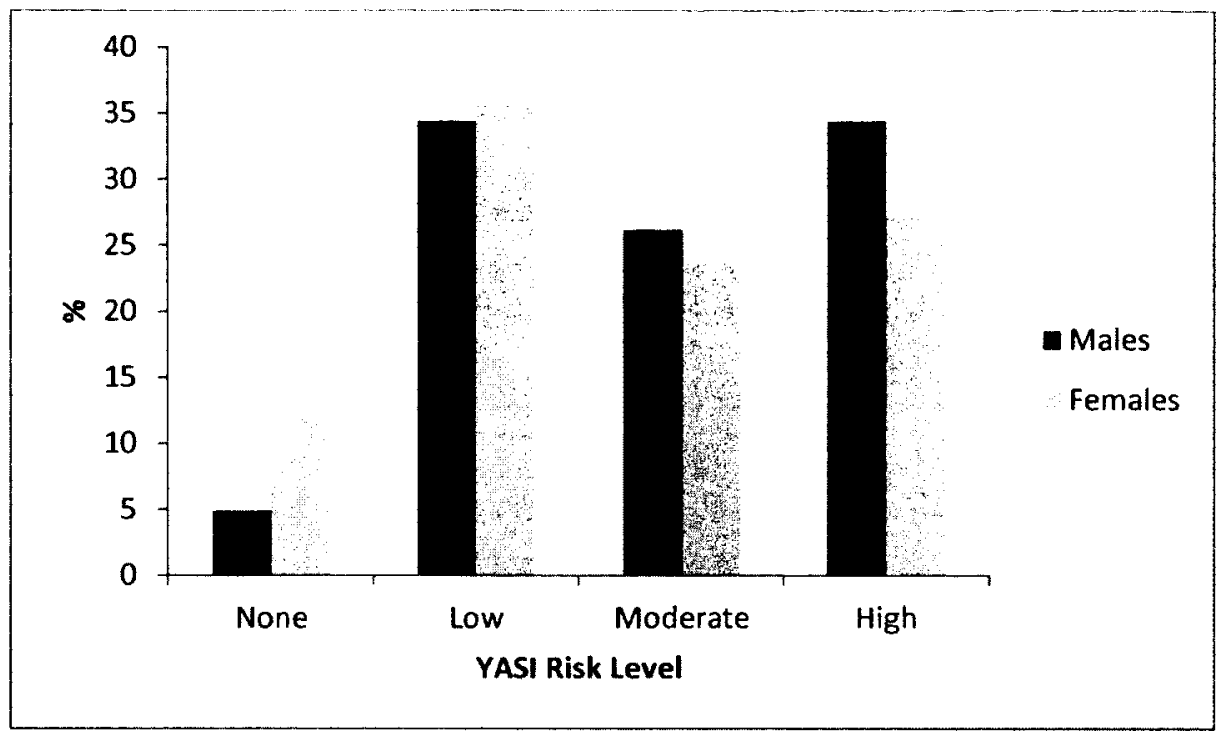

Figure 17. Distribution of Youth for YASI Education Domain Dynamic Protective Levels

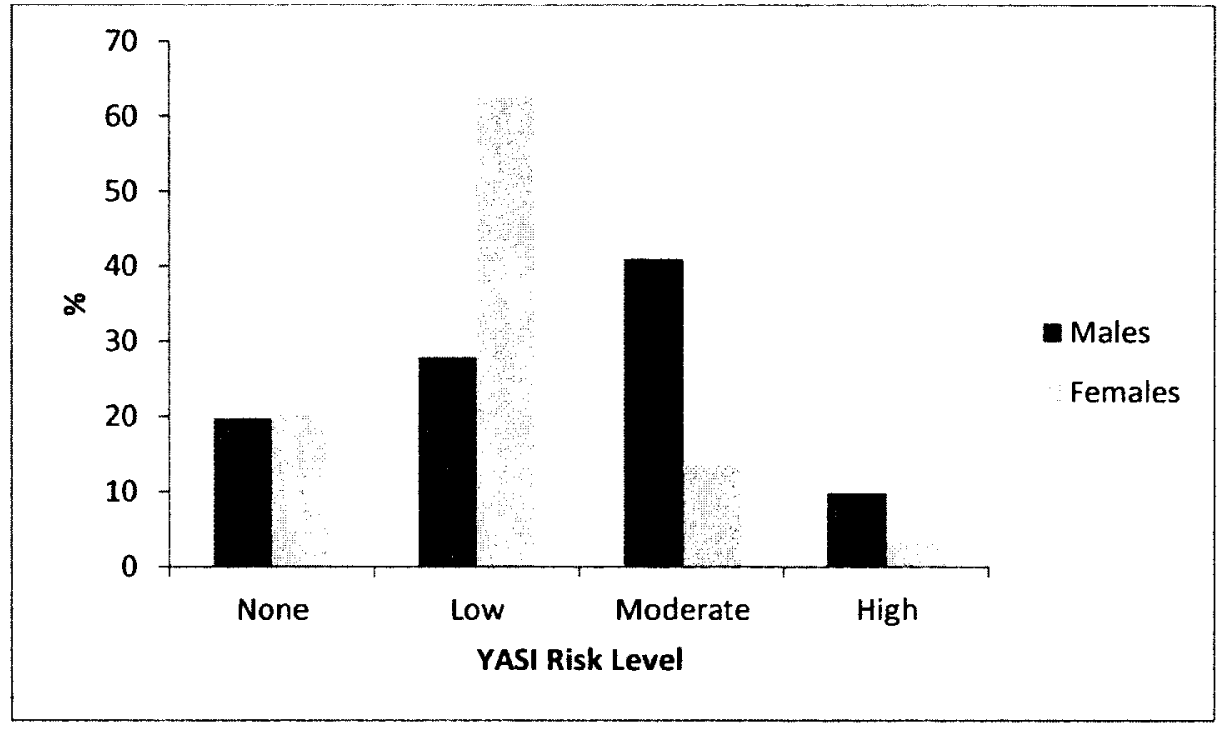

Figure 18. Distribution of Youth for YASI Social Domain Dynamic Protective Levels 


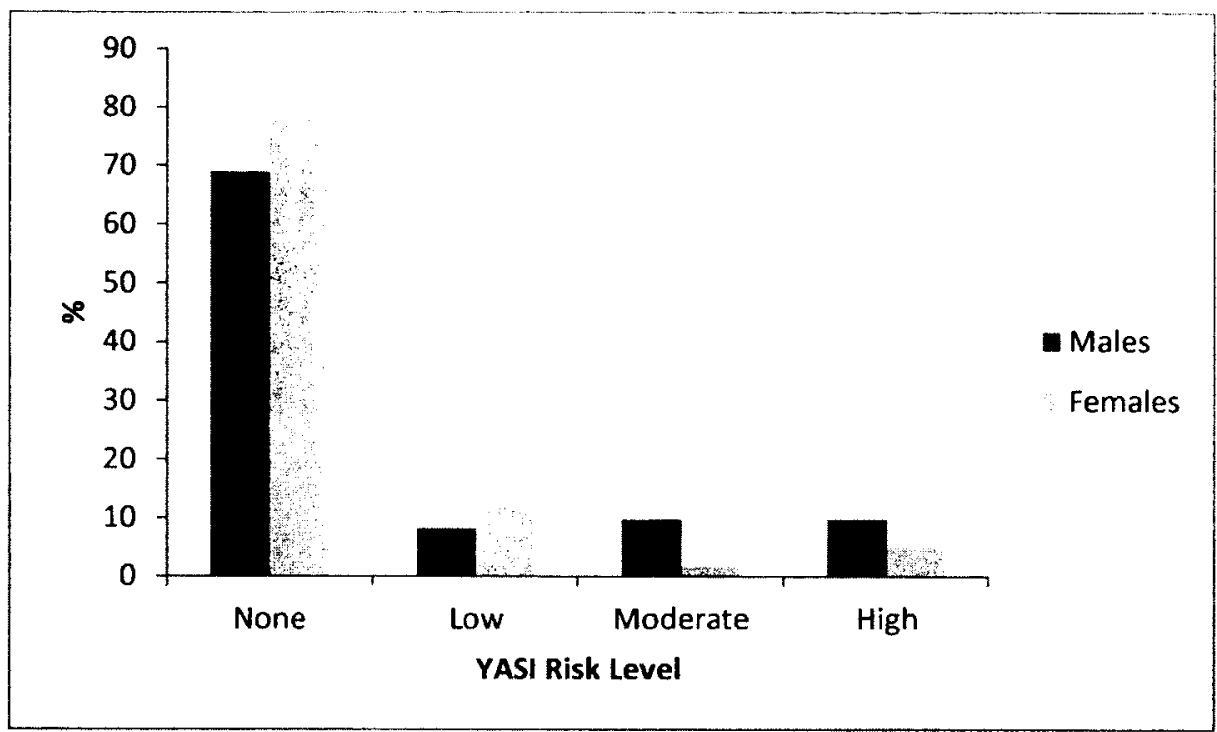

Figure 19. Distribution of Youth for YASI Aggression Domain Dynamic Protective Levels

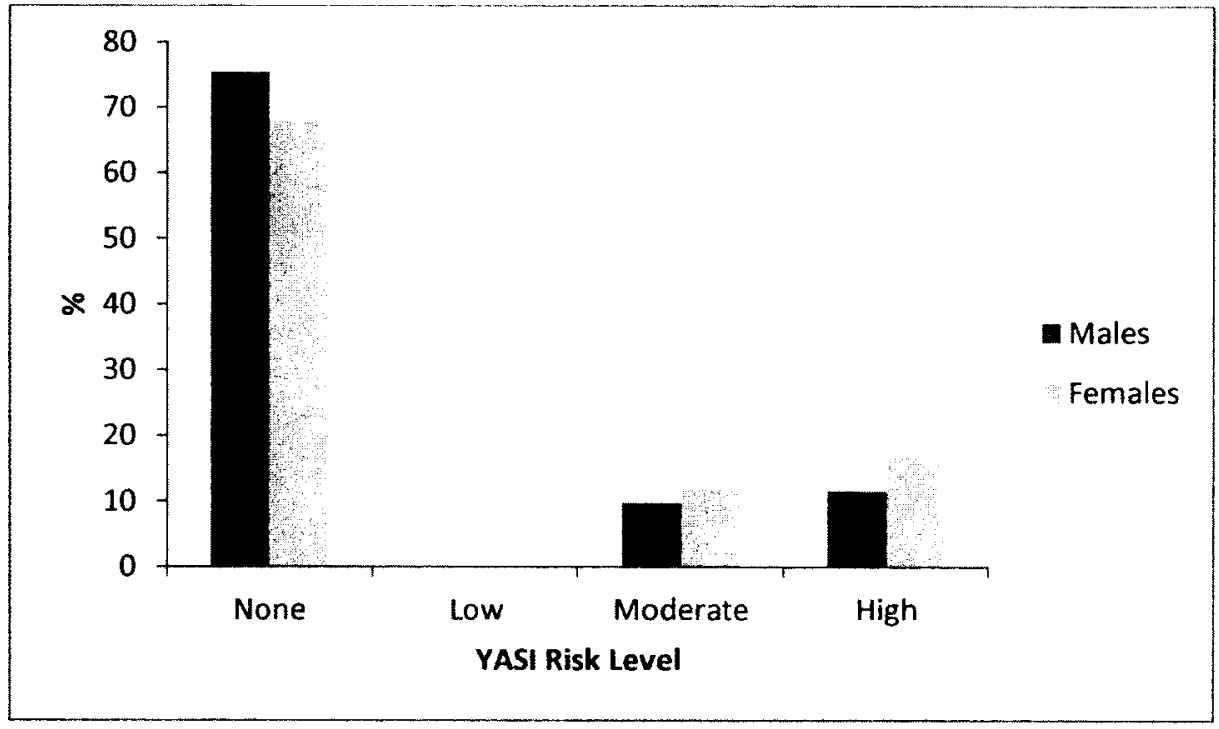

Figure 20. Distribution of Youth for YASI Attitude Domain Static Protective Levels 


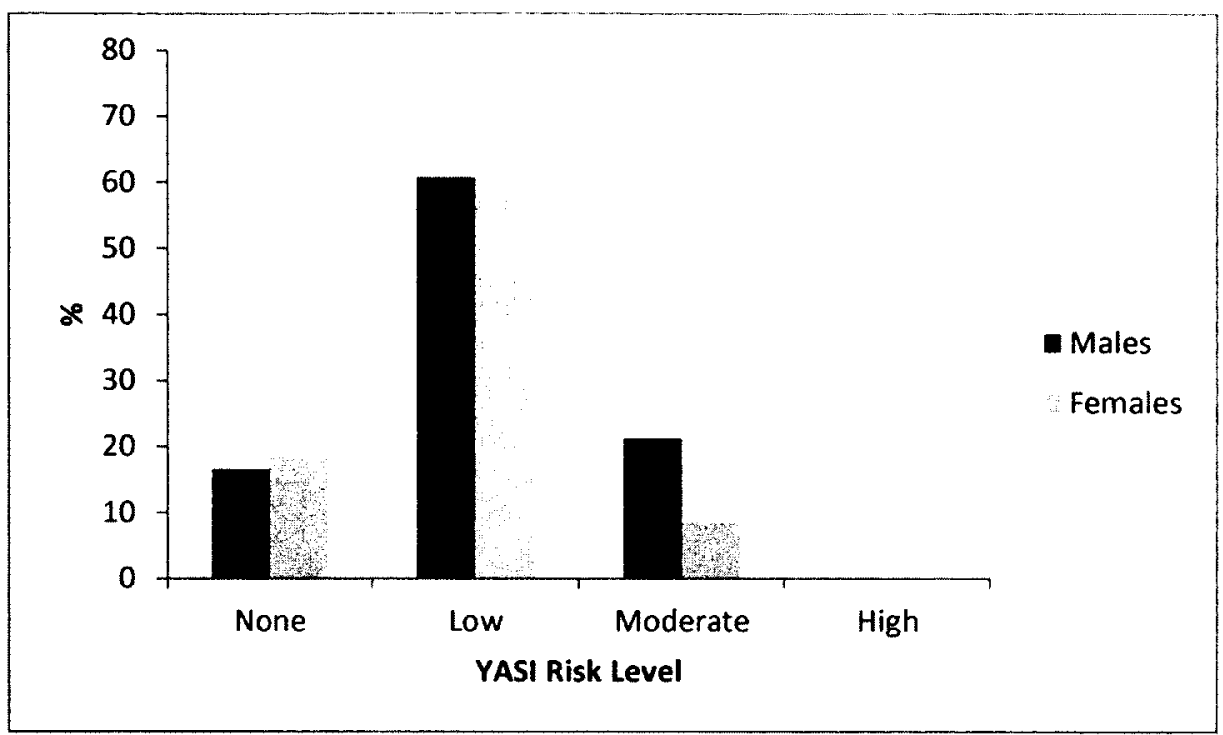

Figure 21. Distribution of Youth for YASI Attitude Domain Dynamic Protective Levels

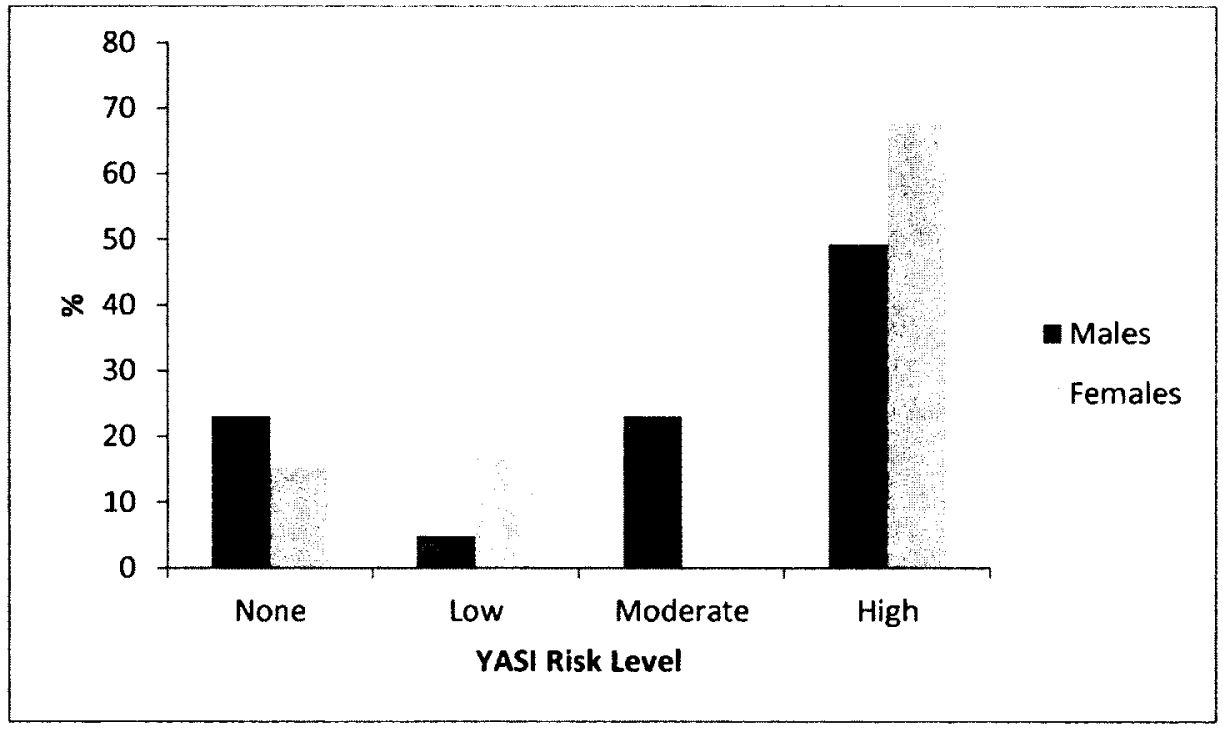

Figure 22. Distribution of Youth for YASI Skills Domain Dynamic Protective Levels 


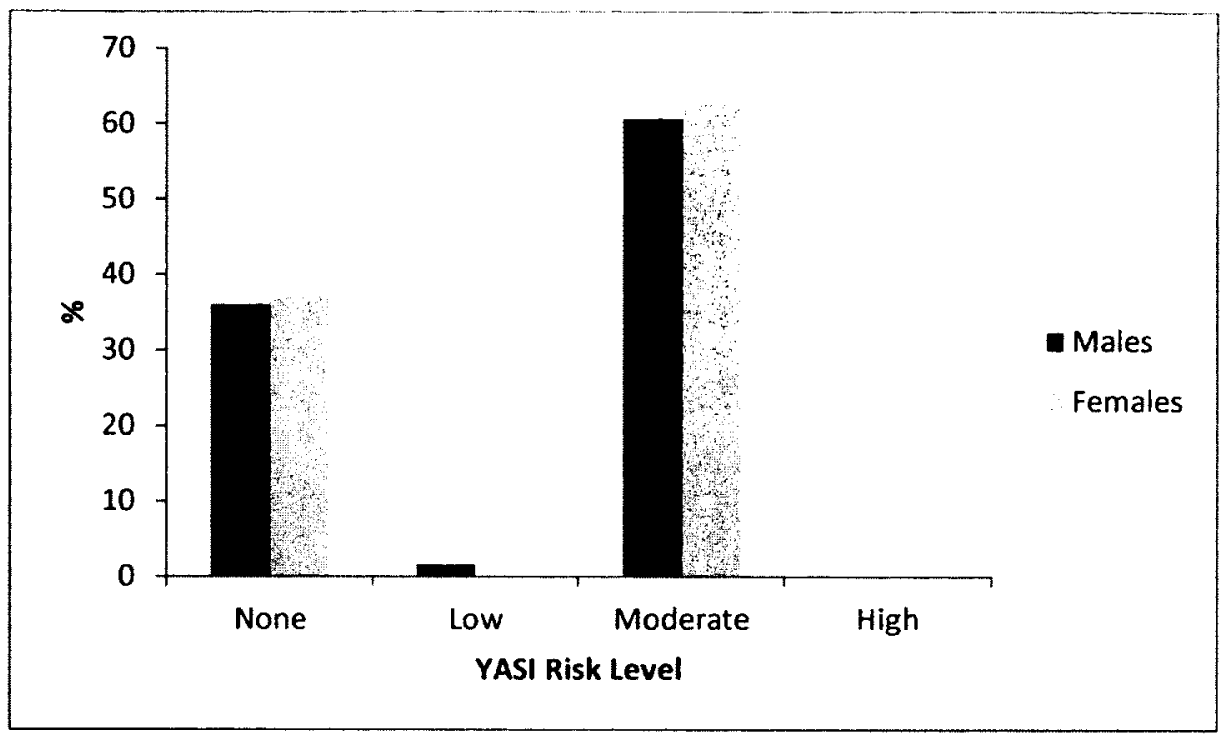

Figure 23. Distribution of Youth for YASI Employment Domain Static Protective Levels

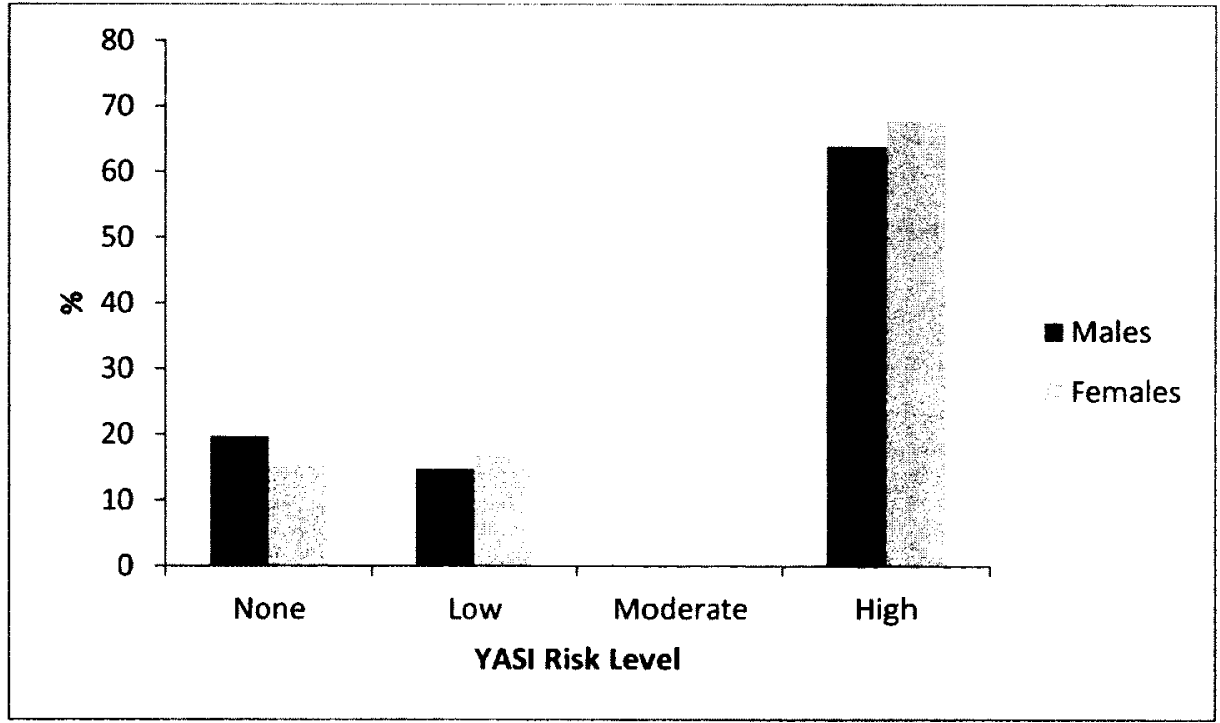

Figure 24. Distribution of Youth for YASI Employment Domain Dynamic Protective Levels 




Figure 25. Distribution of Youth for YASI Total Static Protective Levels

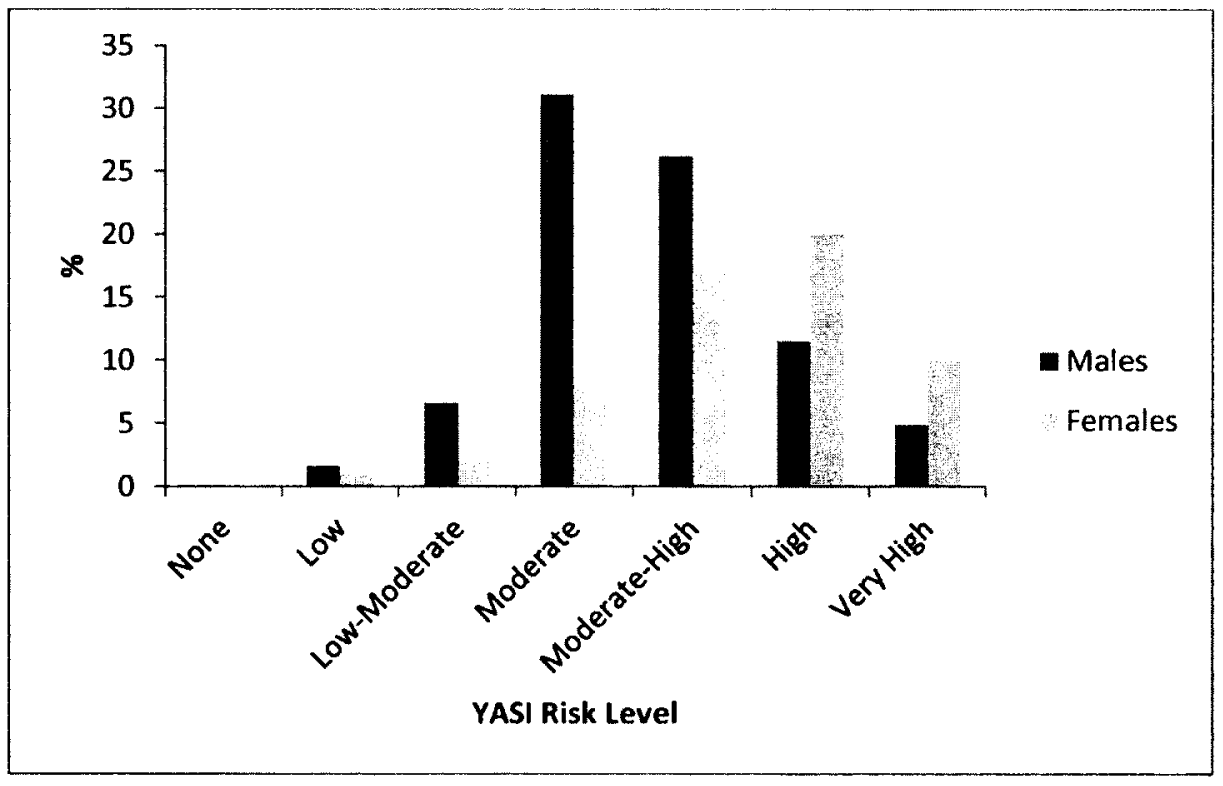

Figure 26. Distribution of Youth for YASI Total Dynamic Protective Levels 
An Exploration of Strengths, Promotive and Protective Factors

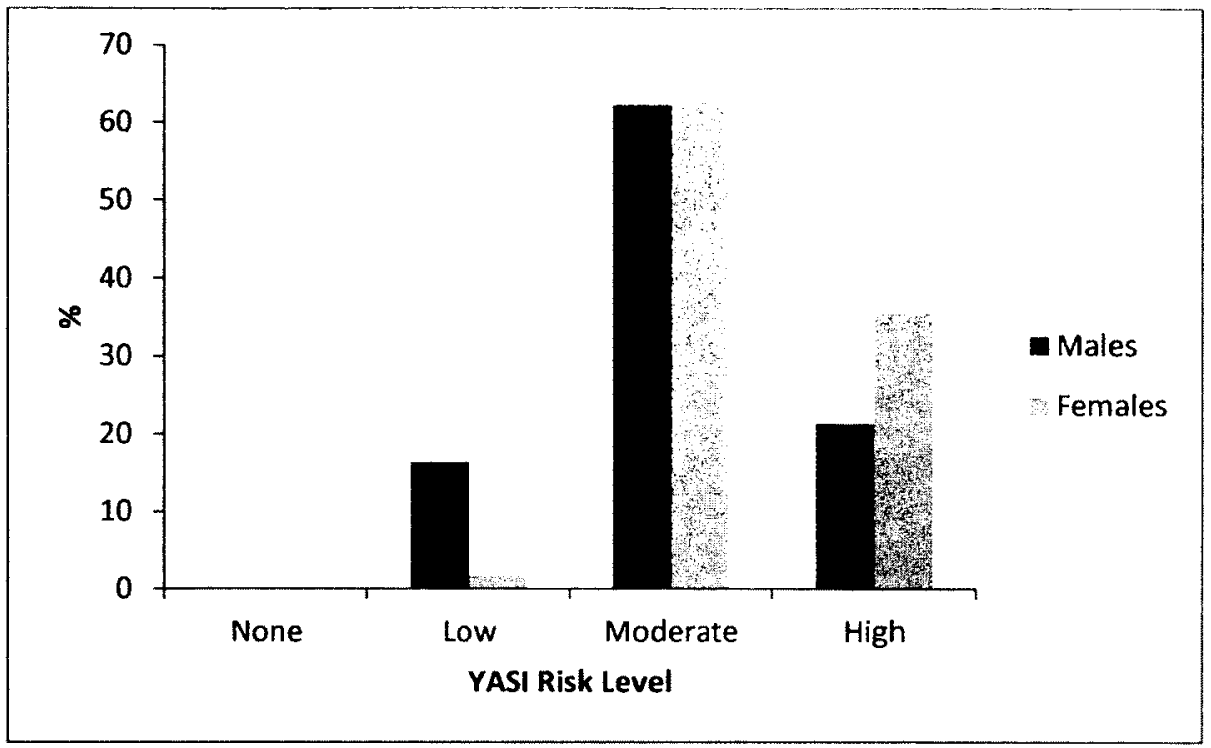

Figure 27. Distribution of Youth for YASI Total Protective Levels

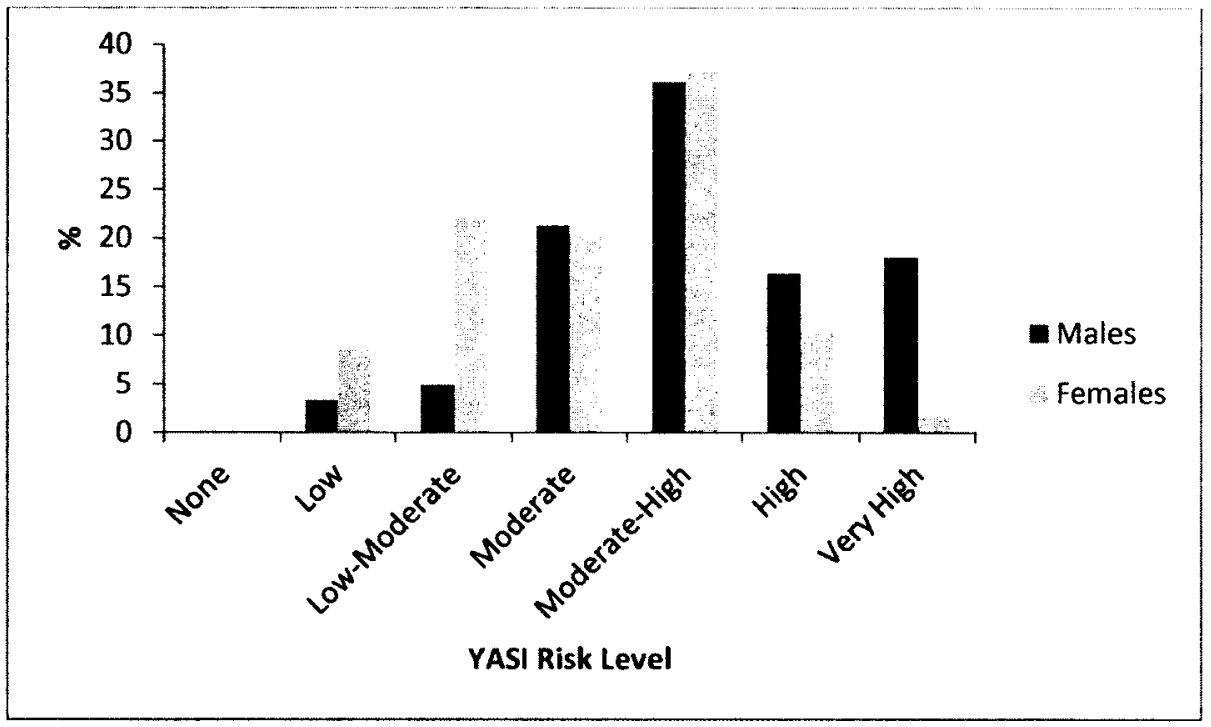

Figure 28. Distribution of Youth for YASI Total Dynamic Risk Levels 


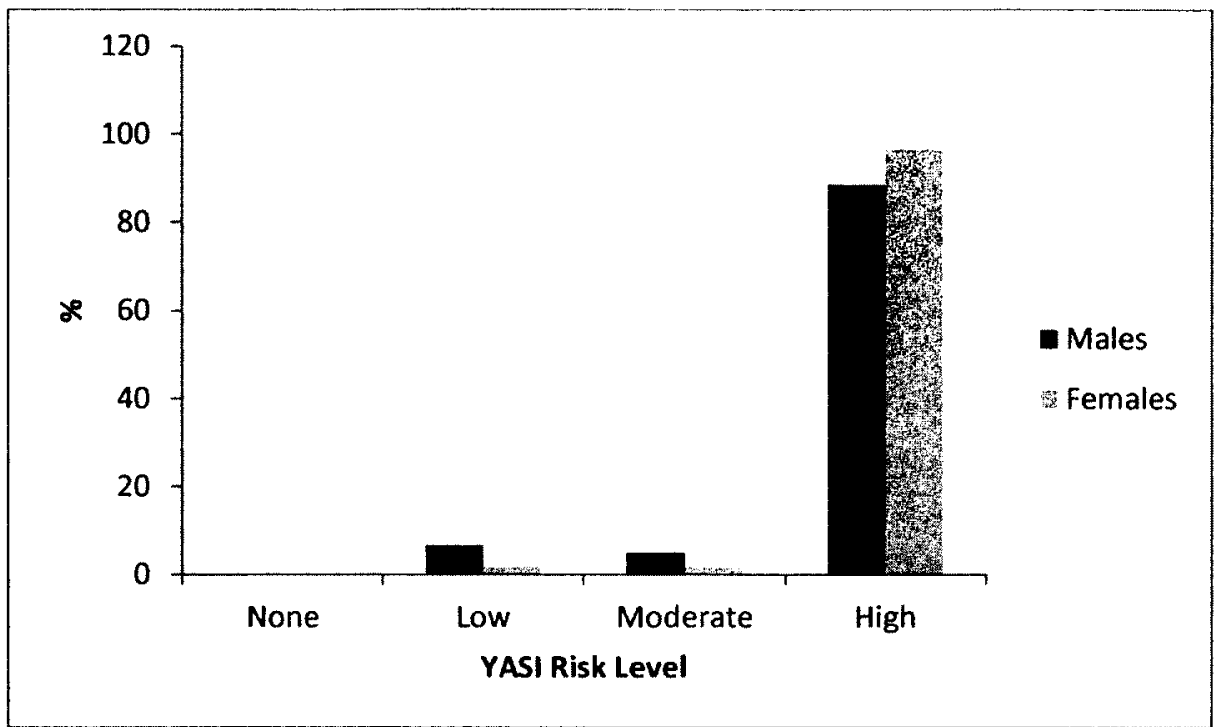

Figure 29. Distribution of Youth for YASI Total Static Risk Levels 